


\section{INSTRUCTIVE MATERIALS FOR TENDON AND LIGAMENT AUGMENTATION}

João Crispim 


\section{Members of the committee:}

$\begin{array}{lll}\text { Chairman: } & \text { Prof. dr. ir. J.W.M. Hilgenkamp } & \text { (University of Twente) } \\ \text { Promotors: } & \text { Prof. dr. D.B.F. Saris } & \text { (University of Twente) } \\ & \text { Prof. dr. ir. P. Jonkheijm } & \text { (University of Twente) } \\ \text { Co-promotor: } & \text { Dr. H. Fernandes } & \text { (University of Coimbra) } \\ \text { Members: } & \text { Prof. dr. H.B.J. Karperien } & \text { (University of Twente) } \\ & \text { Prof. dr. P.C.J.J. Passier } & \text { (University of Twente) } \\ & \text { Prof. dr. D.F. Stamatialis } & \text { (University of Twente) } \\ & \text { Prof. dr. L. Moroni } & \text { (Maastricht University) } \\ & \text { Dr. L.A. Vonk } & \text { (University Medical Centre Utrecht) }\end{array}$

The research described in this thesis was performed within the laboratories of Developmental Bioengineering (DBE) and Bioinspired Molecular Engineering Laboratory (BMEL) from the MIRA Institute for Biomedical Technology and Technical Medicine, the Molecular Nanofabrication (MnF) group from the MESA+ Institute for Nanotechnology, at the Department of Science and Technology (TNW) of the University of Twente.

Instructive materials for tendon and ligament augmentation

Copyright @ 2016, João Crispim, Enschede, the Netherlands.

All rights reserved. No part of this thesis may be reproduced or transmitted in any form, by any means, electronic or mechanical without the prior written permission of the author.
ISBN:
978-90-365-4207-4
DOI:
$10.3990 / 1.9789036542074$
Cover art:
João Crispim
Printed by:
Gildeprint - the Netherlands 


\title{
INSTRUCTIVE MATERIALS FOR TENDON AND LIGAMENT AUGMENTATION
}

\author{
DISSERTATION
}

to obtain

the degree of doctor at the University of Twente, on the authority of the rector magnificus

$$
\text { Prof. dr. T. T. M. Palstra, }
$$

on account of the decision of the graduation committee,

to be publicly defended

on Wednesday December 7, 2016 at $16.45 \mathrm{~h}$

by

João Crispim

born on October 26, 1989

in Beja, Portugal 
This dissertation has been approved by:

Promotors: Prof. dr. D.B.F. Saris

Prof. dr. ir. P. Jonkheijm

Co-promotor: Dr. H. Fernandes 


\section{Table of Contents}

\begin{tabular}{lll}
\hline Chapter 1: & Scope and outline of the thesis & 1 \\
\hline
\end{tabular}

\begin{tabular}{|c|c|c|}
\hline Chapter 2: & General introduction & 5 \\
\hline 2.1. & Introduction to the problem & 6 \\
\hline \multirow[t]{3}{*}{2.2 . } & Biology of tendons and ligaments & 7 \\
\hline & Function and structure & 7 \\
\hline & Injuries and pathologies & 9 \\
\hline 2.3. & Healing process & 10 \\
\hline \multirow[t]{5}{*}{ 2.4. } & Improving tendon/ligament healing & 12 \\
\hline & Surgical reconstruction & 12 \\
\hline & Administering growth factors & 14 \\
\hline & Augmentation devices & 15 \\
\hline & 2.4.4. & 16 \\
\hline 2.5. & Conclusions and future directions & 19 \\
\hline 2.6. & References & 20 \\
\hline
\end{tabular}

Chapter 3: TGF- $\beta 1$ activation in hamstring cells through 27 growth factor-binding peptides on polymers

3.1. Introduction 28

3.2. Materials and methods 30

3.3. Results 35

3.3.1. Fabrication of $\mathrm{PCL}$ films presenting TGF- $\beta 1$-binding 35 peptides

3.3.2. TGF- $\beta 1$ immobilization on the functionalized PCL films 36

3.3.3. Bioactivity of the immobilized TGF- $\beta 1$ in a TGF- $\beta 1$ reporter 38 cell line

3.3.4. Immobilized hTGF- $\beta 1$ activates the TGF pathway via 39 $\mathrm{Smad} 2 / 3$ in human hamstring cells 
3.3.5. Immobilized hTGF- $\beta 1$ specifically activates TGF- $\beta 1$ target 40 genes

3.3.6. Effects of immobilized hTGF- $\beta 1$ on endogenous collagen 41 production

3.3.7 TGF- $\beta 1$-binding peptide captures native circulating TGF- $\beta 1 \quad 42$ leading to its accumulation on the implanted functionalized polymer

3.4. Discussion

3.5. Conclusions 50

3.6. Acknowledgments 50

3.7. References 51

3.8. Supporting information 53

Chapter 4: Immobilization of hBMP-2 on polymers directs cell 55 fate

4.1.

Introduction

56

4.2. Materials and methods 58

4.3.

Results

62

4.3.1. Functionalization of PCL with BMP-2-binding peptides

62

4.3.2. BMP-2 immobilization on the functionalized PCL films 63

4.3.3. Immobilized hBMP-2 retains its bioactivity 64

4.3.4. Synthesis of ALP in response to immobilized hBMP-2 66

4.3.5. Capture of endogenous BMP-2 by the BMP-2-binding 67 peptide leads to tissue response

4.4. Discussion 70

4.5. Conclusions 75

4.6. Acknowledgments 76

4.7. References 76

$\begin{array}{ll}\text { 4.8. Supporting information } & 79\end{array}$ 


\section{Chapter 5: Improvement of medical devices with VEGF-binding 81}

peptides in order to induce vascularization in orthopaedic injuries

5.1 Introduction 82

5.2. Materials and methods 84

5.3. Results $\quad 88$

5.3.1. Functionalization of PCL with VEGF-binding peptides 88

5.3.2. Immobilization of VEGF onto PCL films 89

5.3.3. Immobilized hVEGF enhances survival of HUVECS 90

5.3.4. In vivo capture of endogenous VEGF by the VEGF-binding 91 peptide leads to the appearance of blood vessel-like structures

5.3.5. Functionalization of orthopaedic medical devices with VEGF-binding peptides allows the immobilization of VEGF

5.3.6. Engineering hVEGF immobilization in functionalized CMIs in order to mimic the meniscus's vascular network distribution

5.4. Discussion 99

$\begin{array}{ll}\text { 5.5. Conclusions } & 103\end{array}$

$\begin{array}{ll}\text { 5.6. Acknowledgments } & 103\end{array}$

$\begin{array}{ll}\text { 5.7. References } & 104\end{array}$

5.8. Supporting information 106

\begin{tabular}{|c|c|c|c|}
\hline Chapter 6: & \multicolumn{2}{|r|}{ Spatially presenting hTGF- $\beta 1$ on biopolymeric films } & 109 \\
\hline 6.1. & \multicolumn{2}{|c|}{ Introduction } & 110 \\
\hline 6.2 . & \multicolumn{2}{|c|}{ Materials and methods } & 112 \\
\hline \multirow[t]{4}{*}{ 6.3. } & \multicolumn{2}{|c|}{ Results } & 115 \\
\hline & 6.3.1. & $\begin{array}{l}\text { Amount of immobilized hTGF- } \beta 1 \text { is dependent on the } \\
\text { reaction time between the PCL films and the TGF- } \beta 1 \text { - } \\
\text { binding peptide }\end{array}$ & 115 \\
\hline & 6.3 .2$. & $\begin{array}{l}\text { Characterization of TGF- } \beta 1 \text {-binding peptide gradients on } \\
\text { PCL films }\end{array}$ & 116 \\
\hline & $6.3 \cdot 3$ & $\begin{array}{l}\text { TGF- } \beta 1 \text {-binding peptide gradients lead to gradients of } \\
\text { hTGF- } \beta 1\end{array}$ & 118 \\
\hline
\end{tabular}


6.4. Discussion 121

6.5. Conclusions 124

$\begin{array}{ll}\text { 6.6. } & 124\end{array}$

$\begin{array}{ll}\text { 6.7. } & 125\end{array}$

$\begin{array}{lll}\text { 6.8. Supporting information } & 127\end{array}$

\begin{tabular}{llr}
\hline Chapter 7: & Final conclusions and future perspectives & $\mathbf{1 2 9}$ \\
\hline 7.1. & Final conclusions & 130 \\
7.2. & Future perspectives & 131 \\
& 7.2.1. $\quad$ Growth factor combination & 131 \\
& 7.2.2. $\quad$ Spatial presentation of growth factors & 131 \\
& 7.2.3. $\quad$ Final considerations & 134 \\
& References & 135 \\
\hline
\end{tabular}

$\begin{array}{ll}\text { Summary } & 137\end{array}$

$\begin{array}{lr}\text { Samenvatting } & 139\end{array}$

$\begin{array}{ll}\text { Acknowledgements } & 141\end{array}$

$\begin{array}{ll}\text { About the author } & 145\end{array}$

$\begin{array}{lr}\text { List of publications } & 147\end{array}$ 


\section{Chapter 1}

\section{Scope and outline of the thesis}

Tendons and ligaments ( $\mathrm{T} / \mathrm{L}$ ) are the connective tissue that connects muscle to bone and bone to bone, respectively. The main function of tendons is to translate muscle contractions into joint motion and consequently generate movement. Ligaments function to stabilize joints and guide them during their range of motion. Injuries to these tissues continue to be a major clinical problem for clinicians, patients and society in general. Injuries to these tissues are always associated with pain, disability and great healthcare costs. Additionally, the current treatment options fail to restore the biomechanical properties of the repaired tissue to those of native tissue. The field of tissue engineering is being explored in order to improve the repair and healing of these damaged tissues; several studies have been conducted investigating the use of biomaterials, cells, bioactive factors or a combination of these. One of the most important groups of bioactive molecules is that of growth factors (GFs). These hormonelike proteins are involved in several cellular processes and play roles in both the development and repair of T/L. The outcome of their use is dependent on the type of GF and their properties, delivery system, microenvironment, target cells and on the clinical situation. However, the use and administration of GFs is currently an engineering challenge, is not clinically feasible and is heavily regulated due to the side effects of their administration. The goal of this thesis is to investigate the functionalization of different biomaterials with GF-binding peptides. We set out to capture and immobilize GFs known to be relevant to $\mathrm{T} / \mathrm{L}$ healing. With this strategy, we aim to capture the endogenous GFs through affinity with the corresponding GF-binding peptide, and immobilize these onto the biomaterial. This allows us to make use of the native GFs that circulate in the body and consequently to overcome the problems associated with GF administration. The ultimate goal is to develop a sleeve functionalized with GF-binding peptides, which can be wrapped around the damaged T/L or graft, in order to capture the endogenous GFs and present them to the damaged tissue (Figure 1.1). Spatial control over the presentation of different GFs on such a sleeve will enhance the healing of different tissues, such as bone, soft tissue and the interface between these. 

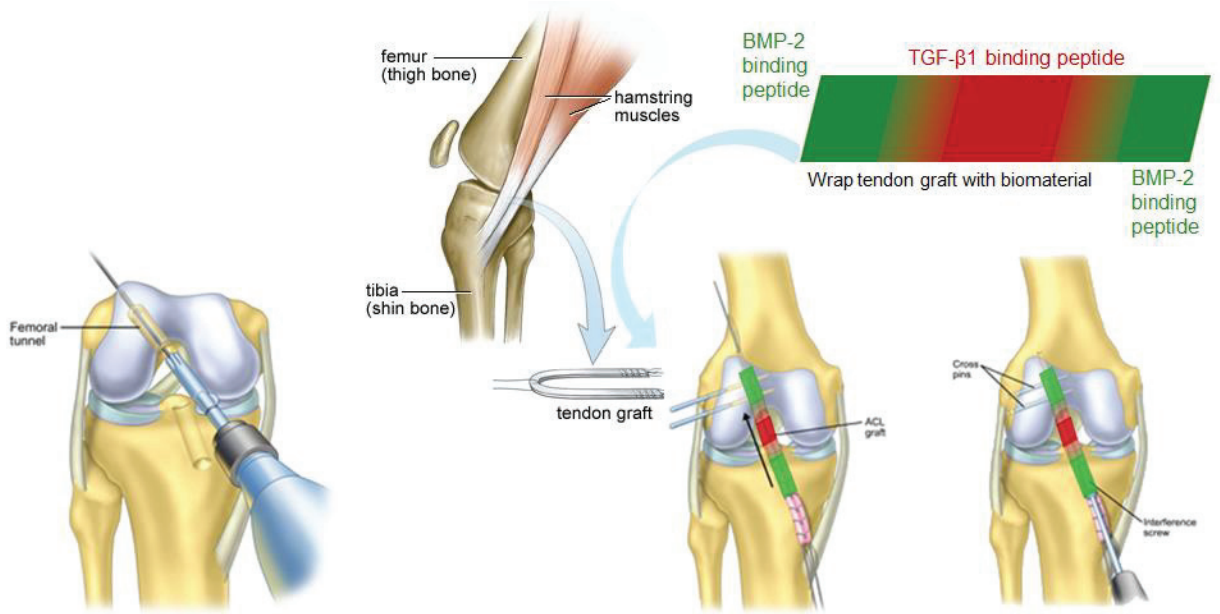

Figure 1.1. Schematic representation of the use of a sleeve, with patterning of GF-binding peptides envisioned for use in $\mathrm{ACL}$ reconstruction.

Chapter 2 provides an overview of the function and structure of $\mathrm{T} / \mathrm{L}$, the most common injuries and their respective surgical intervention procedures. The current strategies explored in order to enhance T/L healing are also discussed with a focus on the use of biomaterials. Finally, the future directions and current challenges of the T/L field are reviewed.

In chapter 3, we study soft tissue healing by investigating the delivery and presentation of transforming growth factor $\beta 1$ (TGF- $\beta 1$ ). The TGF- $\beta 1$-binding peptide was synthetized using FMOC-solid peptide synthesis and characterized with mass spectroscopy and high performance liquid chromatography. The peptide was covalently attached to a polycaprolactone film and its affinity towards TGF- $\beta 1$ assessed by immunochemistry. The bioactivity of the immobilized TGF- $\beta 1$ was investigated using a TGF-responsive cell line and human derived hamstring cells. The production of collagen in response to the immobilized TGF- $\beta 1$ was quantified at the RNA and protein levels. In vivo studies were performed to investigate the potential of the peptide in capturing endogenous TGF- $\beta 1$ and to determine whether accumulation of the GF induced a tissue response.

The effect of delivering bone morphogenetic protein 2 (BMP-2) to enhance bone healing is investigated in chapter 4. The BMP-2-binding peptide was synthetized and characterized as mentioned above. Its affinity towards BMP-2 was determined using immunochemistry. A BMP-responsive cell line was used to study the bioactivity of the immobilized BMP-2 and the production of alkaline phosphatase in $\mathrm{C}_{2} \mathrm{C}_{12}$ quantified. 
Subsequently, we studied the behaviour of a biomaterial functionalized with a BMP-2binding peptide for its biological response in vivo.

Presentation of vascular endothelial growth factor (VEGF) in order to promote vascularization is assessed in chapter 5. The VEGF-binding peptide was synthetized, characterized and its affinity towards VEGF confirmed. Proliferation and survivability of human umbilical vein endothelial cells (HUVECs) in response to the immobilized VEGF was investigated. The effect of implanting polycaprolactone films was also assessed through immunochemistry against VEGF and histological staining of the surrounding tissue. Various clinically-applied medical devices were functionalized with the VEGFbinding peptide and the capture of VEGF by these devices was determined using immunochemistry. A strategy to spatially control the presentation of VEGF in the collagen meniscus implant was developed and investigated with immunochemistry.

In chapter 6, a dip coating strategy is used to generate gradients of TGF- $\beta 1$-binding peptide on polycaprolactone films in order to mimic the interface between bone and soft tissue. The peptide gradient was validated with X-ray photoelectron spectroscopy. The profile and spatial presentation of the immobilized TGF- $\beta_{1}$ was evaluated with immunochemistry and quantified with ImageJ. The influence of parameters such as peptide concentration and dipping out speed on the peptide gradient and ultimately on the immobilized TGF- $\beta 1$ spatial distribution was investigated.

The final conclusions and future perspectives for the technology described in this thesis are presented in chapter 7 . 


\section{Chapter 2}

\section{General introduction}

In this chapter, important aspects of the healing of tendons and ligaments $(T / L)$ are reviewed. First, the biology of these tissues and the most common injuries are summarized. Next, surgical interventions for repair and reconstruction are described and the healing process and outcome are discussed. We conclude with a discussion of current repair/reconstruction interventions and strategies regarding how to improve the healing process using these interventions. 


\subsection{Introduction to the problem}

Owing to an aging population and involvement in physical activities, musculoskeletal injuries are among the most common injures worldwide. From the 33 million injuries reported in the US per year, approximately $33 \%$ involve $T / L$ [1]. The most frequently reported injury is that of the anterior cruciate ligament $(A C L)$ tear or rupture, which accounts for more than 80,000 cases per year in the US alone, with an estimated cost of US\$1.0 billion [2]. Due to improvements in quality of life and the increasing participation of the population in physical activities, the incidence of these injuries is likely to rise, affecting patient quality of life and increasing healthcare costs. Injuries to these tissues are always associated with pain, swelling and disability, with the extent of the damage dictating recovery time $[3,4]$. When tears or ruptures of the tissues occur, surgical intervention is usually needed. The main goal of this surgical treatment is to stabilize and restore normal movement to the joint [5]. Due to the nature of these tissues and their inherent poor healing capacity, surgical intervention is also needed to direct the natural healing process. However, even with the available treatments, complete healing of the damaged tissue is difficult to achieve, which can ultimately lead to scarring, restrictions to the range of motion, stiffness/weakness of the joint, improper healing and re-injury [6]. After surgery, a long recovery period is required of between 9 to 12 months, during which the patient's movement and quality of life are affected and the pre-injury properties of the $T / L$ are likely not yet fully restored [7]. Consequently, there is a need to improve the current treatments and make the overall surgical and rehabilitation process more efficient, shorter and friendlier to the patient.

In the following section, further details of the biology of T/L are described. A section then follows in which the different stages of the healing process are described, and finally the various directions taken for the improvement of the healing process are discussed. 


\subsection{Biology of tendons and ligaments}

\subsubsection{Function and structure}

Tendons connect muscle to bone and transmit forces from muscle contractions to bone, consequently maintaining posture and generating body movement [8]. Ligaments are connective tissue, like tendons, connecting bone to bone [9]. Their main function is to stabilize joints and help guide these joints during their range of motion [10]. Both tissues are composed of cells within an organized extracellular matrix (ECM) largely composed of collagen. Collagen molecules assemble in a helical shape to form fibrils (Figure 2.1), which in turn give origin to fibres, fascicles and ultimately to a functional T/L [8]. Collagenous proteins account for $70-80 \%$ of the dry weight of $\mathrm{T} / \mathrm{L}$, elastin between $1-2 \%$ and the remaining is comprised of glycoproteins, proteoglycans and water [11]. Type I collagen is the main constituent of the ECM, followed by type III collagen. Other types of collagen that contribute to the basic structure of T/L can be found, such as types II, $\mathrm{V}$ and $\mathrm{XI}$. Type $\mathrm{V}$ collagen, for instance, controls the initiation of collagen fibril assembly [12]. Elastin is critical for the elasticity and stability of these tissues [13]. Proteoglycans such as decorin, aggrecan and versican are also present in the composition of T/L, playing key roles in the structural integrity and organization of collagen networks [14]. Tenomodulin and CD44 are two examples of glycoproteins found in T/L [15]. CD44 is an important player during the healing process, while tenomodulin is involved in tendon development $[16,17]$. Water is believed to reduce friction [1]. Despite their similar architecture, there are some differences between $\mathrm{T} / \mathrm{L}$ in terms of type and proportions of collagen, orientation and organization of the collagen proteins and non-collagenous protein content [18]. Ligaments, compared to tendons, have a higher cellularity, a higher content of type III collagen, glycosaminoglycans (GAGs) and water content, and a lower amount of total collagen, making them more metabolically active than tendons [19]. Moreover, the type of cells found in these tissues differ. In tendons, tenoblasts and tenocytes, which are fibroblast-like cells, account for 90-95\% of the cell content, while the remaining $5-10 \%$ includes chondrocytes at the enthesis, synovial cells, and vascular and smooth muscle cells [20]. Tenocytes have an elongated shape, while tenoblasts have a more rounded and spheroid shape [21]. Both cell types are crucial for maintaining tendon homeostasis, as they are primarily responsible for ECM synthesis and assembly [22]. Tenoblasts are classified as an active form of tenocytes, as they have a higher proliferation and apoptosis index than tenocytes. Ligaments are mainly composed of fibroblasts that can have different morphologies depending on their spatial location within the tissue. 
a

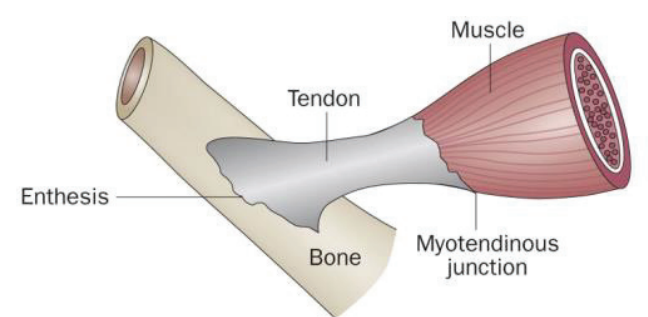

b


Nature Reviews | Rheumatology

Figure 2.1. (a) Schematic of the connection between bone and muscle through tendons. (b) Spatial organization of collagen in tendons. Visualization of tendon collagen fibres using electronmicroscopy (c), histological staining (d) and immunochemistry (e). Reprinted with permission from Nourissat et al. (2015) [8]. 


\subsubsection{Injuries and pathologies}

Injuries to T/L always involve alteration of their highly organized structural architecture. The factors that cause these injuries are classified into intrinsic factors such as age, gender, biomechanics, diseases or genetic factors, and extrinsic factors that include abnormal physical load and environment [23]. Injuries to these tissues are divided into acute injuries, where extrinsic factors are the main cause, and chronic injuries, where intrinsic factors also play an important role [23]. Acute injuries result from one trauma in which an abnormal load was applied to the T/L, whilst chronic injuries result from repetitive overloading. Chronic pathologies develop slowly, are long-lasting or recurring and are characterized by pain, inflammation, and a decrease in strength and range of motion [7]. These types of injuries are more likely to occur in males and in older and obese individuals [22]. The repetitive abnormal mechanical load will induce the production of biological factors that will lead to the remodelling of $T / L$. Such injuries occur without macroscopic changes in the structure of $\mathrm{T} / \mathrm{L}$, but can be identified by changes in collagen organization, in the composition of GAGs or other proteoglycans and noncollagenous proteins, hypercellularity, neovascularization and neural activity, such as an increase in neurotransmitters at the injury site $[8,22]$. Chronic injuries are treated with rest, the use of ice packs to reduce the swelling and pain, anti-inflammatory drugs to reduce pain and inflammation, eccentric exercise therapy to improve function and decrease pain, and steroid injections or surgical intervention [24]. Acute injuries, on the other hand, are the partial or total rupture of a $T / L$ and compromise the integrity and continuity of the $T / L$ as a whole unit. These include sprains, strains and contusions [25]. Sprains are stretches to the $T / L$ and commonly occur in ankles, knees and wrists. Strains also involve stretches within the muscle and T/L or a partial/total tear of the muscletendon combination, and are more likely to occur in the foot, leg or back. Surgery and physiotherapy are the main treatments for these types of injuries, while in the case of total rupture, full reconstruction of the $\mathrm{T} / \mathrm{L}$ with grafted tissue is needed. The main goal of the treatments for both chronic and acute injuries is to reduce pain and restore $\mathrm{T} / \mathrm{L}$ range of motion; however, this can only be partially achieved since the biomechanical properties of the pre-injury tissue are never fully restored. 


\subsection{Healing process}

Upon T/L injury, the natural healing process is triggered (Figure 2.2). This healing process comprises three stages: inflammation, proliferation or repair and remodelling $[7,8,23$, 26]. The healing process differs between tissues, as some ligaments, such as the medial collateral ligament $(\mathrm{MCL})$, have good healing potential, whereas the $A C L$ has a very low healing capacity (Figure 2.3) [27].

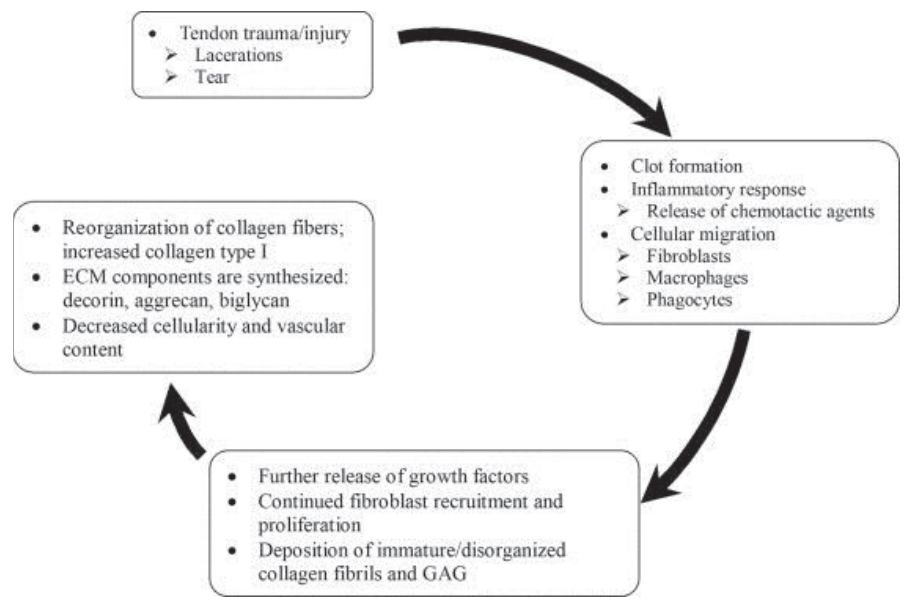

Figure 2.2. Healing process of an injured tendon. Reprinted with permission from James et al. (2007)[1].


Figure 2.3. Differences in the healing response of the $A C L$ and the MCL. Reprinted with permission from Kiapour et al. (2014) [28]. 
Upon injury, the blood vessels present in the T/L rupture and signalling molecules are released by intrinsic cells [29]. The release of these molecules attracts several cell types, initially red and white blood cells and ultimately platelets, leading to the formation of a fibrin clot around the wound [1,29]. The formation of this clot leads to the release of chemotactic factors, such as growth factors (GFs). GFs attract other cell types to the wound site. Macrophages consume the tissue debris by phagocytosis and fibroblasts start migrating to the injury site. This stage lasts from hours to a few days post injury, where the increase in cell number and initiation of a vascular network provides stability and continuity at the injured site [7]. The migration of fibroblasts initiates the second stage, called the proliferative stage. The arrival of fibroblasts and the release of more GFs from macrophages triggers the proliferation and production of ECM components by the fibroblasts [7]. This stage is therefore also characterized by the migration of extrinsic fibroblasts and the proliferation of intrinsic fibroblasts. This high cellular activity leads to the deposition of collagen, proteoglycans and other ECM molecules. These components are initially randomly organized in the ECM, where type III collagen is the main constituent [1]. This stage is initiated a few days after injury and can last up to one to two months [7]. The third phase, called the remodelling phase, is characterized by a decrease in cellularity, the synthesis of type III collagen, the development of a vascular network and an increase in type I collagen content $[1,30]$. During this stage, the collagen fibres become organized and spatially orientated. This phase can last up to one year; however, the repaired tissue will never regain its pre-injury biomechanical properties [7]. After the healing process, the diameter and crosslinking of the collagen fibrils is inferior compared to the non-injured tissue, leading to a weaker tissue that is more prone to re-injury and further damage.

All the molecular events that occur during T/L healing are orchestrated by GFs. Several GFs are involved in this process, having key roles in different cellular processes at different stages. Transforming growth factor $\beta$ (TGF- $\beta$ ) is known to be active during the entire healing process. Its expression levels increase during the inflammatory stage, immediately after the injury. This GF is produced and released by platelets, macrophages, fibroblasts and other cell types [31-33]. TGF- $\beta$ induces the recruitment of cells during the inflammatory stage $[1,34]$, stimulates the synthesis of ECM components and type III collagen during the repair stage [35] and, during the remodelling stage, is involved in the termination of cell proliferation and induction of type I collagen synthesis and secretion [35]. Another key GF is insulin-like growth factor 1 (IGF-1), which is also active during all stages of $\mathrm{T} / \mathrm{L}$ healing. During the inflammatory stage, it recruits fibroblasts and inflammatory cells to the wound. In the repair stage, it is involved in cell proliferation and 
the synthesis of collagens and ECM components [36, 37] and ultimately, during the final stage, it contributes to ECM remodelling [1]. Basic fibroblast growth factor (bFGF) is also found in injured tendons [38]. This GF is involved in the formation of the blood vessel network during the inflammatory stage and induces proliferation and synthesis of collagen and ECM components during the repair stage [39-41]. An important GF during the initial inflammatory stage is platelet derived growth factor (PDGF). This GF is released by platelets immediately after injury, is involved in the attraction of other cells types to the injury site, and in the proliferation and production of other GFs, such as IGF-1 and TGF- $\beta 1$ [42-44]. Vascular endothelial growth factor (VEGF) is also crucial during the healing process as it is involved in the formation and maintenance of the vascular network [45, 46]. VEGF also plays a role during the remodelling stage [47]. Bone morphogenetic proteins (BMPs) are also involved in the healing of $\mathrm{T} / \mathrm{L}$ and are crucial to re-establish the interface between soft tissue and bone. BMP-12, -13 and -14 are involved in cell proliferation and type I and III collagen synthesis during $\mathrm{T} / \mathrm{L}$ healing [48, 49], whereas BMP-2, -4 and -7 are powerful inducers of osteogenesis [50].

\subsection{Improving tendon/ligament healing}

\subsubsection{Surgical reconstruction}

As mentioned above, the healing capacity differs between tissues. In a T/L with a poor healing capacity that cannot repair itself, such as the ACL, surgical reconstruction is the most common treatment (Figure 2.4). If left untreated, ACL injuries can lead to loss of function, additional injuries to other structures of the joint (e.g., meniscus) or to the development of other pathology, such as arthrosis [51]. During ACL reconstruction, small incisions are made around the knee and sterile saline is pumped into the knee to expand it and wash out the blood. Next, the surgeon inserts an arthroscope containing a camera into one of the incisions to visualize the inside of the knee and the extent of the damage. The damaged ACL is partially removed and the health of the surrounding tissue checked. Small tunnels are drilled into the femur and tibia through which the graft will be passed and anchored. The next step involves harvesting a graft to replace the damaged ACL. Here, several options are available to the surgeon. The surgeon can select an autograft, most often obtained from the patellar or hamstring tendon, or an allograft, obtained from a deceased donor. The patellar graft was the most used graft for $A C L$ reconstruction in previous decades due to its superior fixation, proper ultimate strength and stiffness, relative ease of harvest, structural similarities with the ACL and possibility of bone-to-bone healing [52]. The graft is harvested from the patellar tendon with bone 
blocks from the tibia and femur at the ends of the tissue. Recently, the hamstring graft has become more popular for ACL reconstruction. This method has several advantages when compared with the patellar graft, including avoidance of damage to the extensor mechanism, reduced quadriceps weakness, lower morbidity associated with the harvesting procedure and easier preparation of the graft. In addition, hamstring grafts provide higher structural properties, replicating the non-isometric behaviour of the native ACL [52]. The selected graft is pulled through the tunnels and held in place with screws or staples. The incisions are closed with stitches or tape, the knee bandaged and the patient allowed to recover. The goal of such an intervention is to restore stability to the knee and prevent further damage to other knee structures. However, in addition to the poor mechanical properties compared with the pre-injured tissue, this procedure is not $100 \%$ successful and often leads to donor site morbidity, a limited range of motion, knee pain and instability, and development of other pathologies such as osteoarthritis (OA) $[51,53]$. Additionally, the long term performance of this intervention depends on several factors including the structural properties of the graft, graft tension, the intraarticular position of the graft and its fixation. However, integration of the graft's soft tissue into the bone remains the major hurdle. Accelerated and proper integration of the soft tissue into the bone tunnels is mandatory for successful ACL reconstruction, requiring bone-to-bone and tendon-to-bone integration when using patellar grafts and tendon grafts, respectively. Taken together, the abovementioned issues can potentially lead to the re-injury of the graft and, consequently, to additional surgeries. 



Figure 2.4. ACL reconstruction procedure [54]. In summary, the damaged ACL is first removed by the surgeon and possible additional damage to the surrounding tissue is assessed. Two tunnels are drilled, one through the tibia and one through the femur. The harvested graft is then inserted into one tunnel and pushed through the other. Finally, the graft is held in position through the use of pins and screws.

\subsubsection{Administering growth factors}

Due to the role of GFs in the healing process, several studies have focused on the effect of their administration in T/L healing [55]. GFs that promote bone or cartilage formation are particularly attractive to enhance integration of the graft into the bone tunnels [56]. Spindler et al. (1996) showed that culturing explants of $A C L$ and patellar tendon from sheep knees with TGF- $\beta 1$ induced cell proliferation [57]. Injections of PDGF into ruptured rat and rabbit ligaments led to an increase in the strength and breaking energy when compared to ligaments in the control group [58-60]. Likewise, injections of bFGF resulted in an increase in cell proliferation and type III collagen expression in defect rat patellar tendon [40]. Similarly, the application of bFGF to defect canine ACL led to an increase in 
neovascularization and granulation tissue [61]. Despite their importance during the healing process, the administration of GFs to injured T/L remains a clinical challenge. The efficacy of GF treatment depends on their precise mechanism of action, temporal and spatial demand, physiological concentration and delivery system. Despite BMP-2 and -7, no other GF is clinically approved, and even these are involved in controversy. However, there is an autologous clinically-approved treatment for improving $T / L$ healing. This treatment is based on platelet-rich plasma (PRP), which is blood plasma that has been enriched with platelets. PRP contains several different GFs, such as PDGF, TGF, VEGF, IGF and other proteins, which are key players in the healing process [62]. PRP is obtained by centrifuging the patient's blood and is therefore inherently safe. Despite the fact that several studies have shown the benefits of using PRP in in vitro and in vivo models of $\mathrm{T} / \mathrm{L}$ repair, its use remains controversial due to the variability of the results obtained [34], likely due to the lack of standardized methods for its preparation. In conclusion, the efficient use of GFs to boost $\mathrm{T} / \mathrm{L}$ reconstruction may only occur in the distant future.

\subsubsection{Augmentation devices}

Despite the success of using biological grafts for $A C L$ reconstruction, their use is associated with several limitations, such as donor site morbidity, limited availability and the transmission of pathogens [55]. One proposed alternative was to use synthetic grafts made from non-degradable polymers. The first reported use of a synthetic graft for ACL reconstruction was in 1918 by Alwyn-Smith [63], who used silk sutures that failed three months post-surgery. Polymers such as polyethylene, polypropylene and polyester were the primary choices as synthetic graft substitutes. Although achieving early successful results, the use of synthetic grafts did not lead to successful long-term results and was often associated with implant failure. Clinical factors such as continuous inflammation in response to the synthetic graft, disorganization of the collagen fibres, granulomatous response to the synthetic graft and degeneration of the cartilage prevented the clinical success of these strategies [64-66]. A second strategy involved combining synthetic grafts with biological tissue from $\mathrm{T} / \mathrm{L}$; however, this yielded weaker constructs [55]. More recently, biodegradable polymers have begun to be employed in engineering $\mathrm{T} / \mathrm{L}$ repair and reconstruction. Due to the clinical history and $\mathrm{T} / \mathrm{L}$ composition, collagen was the primary choice. For example, Dunn et al. (1995) and Bellincampi et al. (1998) seeded high-strength degradable collagen fibre scaffolds with rabbit fibroblasts $[67,68]$. Although the seeded fibroblasts showed proliferation and survival both in vitro and in vivo, these implants never matched the mechanical strength of native ligaments. Some studies have tried to improve the mechanical properties of 
collagen based grafts, but these have also never reached the strength of the native $A C L$ [62]. Other materials, such as alginate, chitosan and hyaluronic acid have faced the same limitations of collagen-based grafts regarding mechanical properties [62]. However, Panaz-Perez et al. (2013) showed that a combination of silk and collagen led to the fabrication of a scaffold that met the minimal mechanical characteristics required for use in ACL reconstruction [69]. More recently, Fanggang et al. (2015) compared the use of a collagen-silk graft with an autologous graft in the ACL reconstruction of a rabbit model [70]. They showed that in the collagen-silk graft, tendon-bone healing was superior, with more trabecular bone growth into this graft when compared with the use of an autologous semitendinosus tendon graft. In summary, due to the variability of tissue ingrowth, immature degeneration of the implant, poor maturation of the host tissue and consequently inferior mechanical properties, the use of biomaterials as grafts has not achieved satisfactory results and further research must be done in order to find a suitable candidate to replace biological grafts for ACL reconstruction.

\subsubsection{Cells}

Several cell types are recruited to the injured site during the $\mathrm{T} / \mathrm{L}$ healing process, as explained above. However, whether the number of cells that migrate to the injury site is sufficient to induce an efficient healing process remains unknown. This has led to the development of cell-based therapies for T/L healing. These cell-based therapies can be divided into three groups: (1) injections of healthy exogenous cells into the injured site in order to replace the damaged cells; (2) the use of biomaterials incorporating cells; and (3) the use of stem cells that produce and release cytokines and GFs to stimulate the healing of $\mathrm{T} / \mathrm{L}$ and that are capable of differentiating into the target cell type [71]. The ideal cell source should be easy to harvest and to expand in vitro, have good proliferation potential, and have the capacity to produce ECM components and to organize them in the matrix according to the $T / L$ structure [71-73]. Several cell sources have been explored in order to promote T/L healing, such as differentiated and/or pluripotent and multipotent cells. Fibroblasts and tenocytes were the first choice due to their presence in these tissues [72]. They were injected into the tissue defects or seeded onto biomaterials and implanted in the defects (Figure 2.5). 


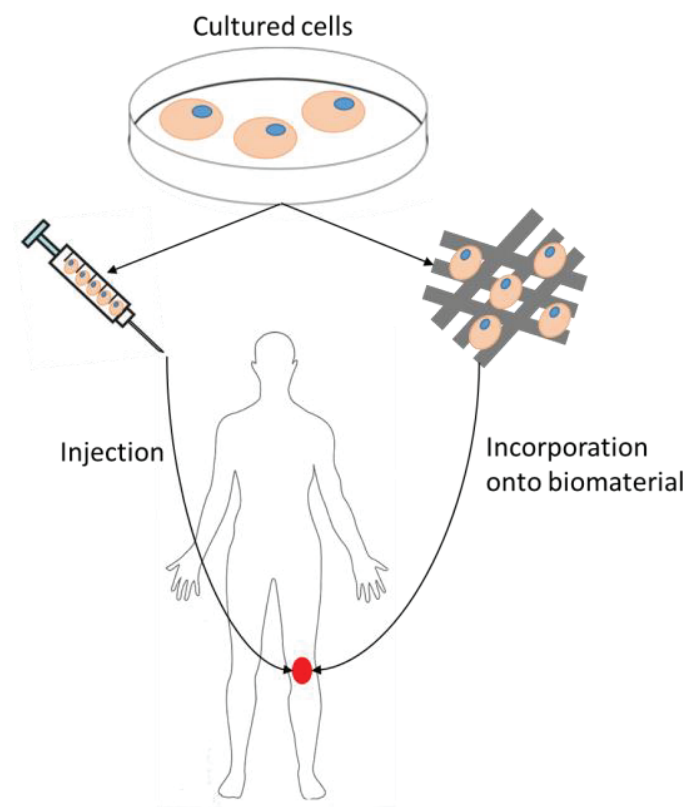

Figure 2.5. Delivery strategies of cells to damaged T/L. Cells are expanded in vitro and administrated to the wound through soluble injections or incorporated onto delivery systems such as biomaterials.

Liu et al. (2006) engineered tendons made of polyglycolic acid (PGA) fibres seeded with either dermal fibroblasts or tenocytes [74]. These artificial tendons were used to repair defects in porcine flexor digital superficial tendons. They showed that these engineered tendons shared a similar strength, which was approximately $75 \%$ of natural tendon strength, and were histologically indistinguishable from the natural tendon. The artificial tendons without cells had a disorganized matrix and were mechanically inferior to the cell-engineered constructs. Another study showed that the injection of autologous tenocytes improved the clinical function and structural repair of the tendinopathy for up to five years in patients with chronic lateral epicondylitis who underwent unsuccessful nonsurgical treatment [75]. An injection of skin fibroblasts proved to be useful in shortterm treatment of patellar tendinopathy by reducing pain and improving function when compared with injections containing only plasma [76]. However, there are some notable limitations to the use of these cells. Tenocytes have a limited capacity to replicate and differentiate [77], their phenotype and function is lost during in vitro expansion [78, 79] and tendon-related markers, such as tenomodulin and thrombospondin 4 , are rapidly downregulated when the tenocytes are cultured in monolayers or organs [80]. Finally, tendons have very few cells; therefore, the number of available cells is rather limited for cell-based therapies [77]. Fibroblasts can form an alternative to tenocytes because of 
their abundance in the human body; however, these are not tendon-specialized cells and are not involved in maintaining tendon homeostasis.

One alternative to differentiated cells is the use of multipotent cells such as bone marrow stromal cells (BMSCs). These cells have good proliferative and metabolic capacities, can differentiate into various lineages and can easily adapt to different environments [72, 73]. Ge et al. (2005) showed that BMSCs have both a higher proliferation and increased collagen production than $A C L$ and $M C L$ fibroblasts, and can survive for at least six weeks in knee joints [81]. Similarly, when cultured on poly(Llactide/glycolide) scaffolds, BMSCs showed higher DNA and collagen production than scaffolds seeded with ACL fibroblasts [82]. MSCs were also reported to have a higher proliferation rate, increased survival and mRNA expression, and an increased protein synthesis of tendon-related markers such as tenascin C and type I and III collagen than ACL fibroblasts when seeded onto silk scaffolds [83]. Hankemeier et al. (2009) showed that fibrin matrix seeded with BMSCs promoted the healing of rat patellar tendons, whereas fibroblasts induced a minor stimulation of the healing process [84]. Other in vivo studies have shown the potential of using scaffolds seeded with BMSCs to repair and regenerate defects in rabbit Achilles' tendons, patellar tendons and flexor profundus tendons [85-87]. Despite these promising results, several concerns have been raised regarding the use of multipotent cells in T/L repair. Award et al. (2003) observed the formation of ectopic bone by collagen scaffolds seeded with BMSCs in defects in rabbit patellar tendons [88]. Similarly, scaffolds seeded with BMSCs formed ectopic bone and expressed alkaline phosphatase when used to repair defects in rabbit tendons [89]. The in vivo use of scaffolds with MSCs was also reported to lead to the formation of tumours [90].

Results from in vitro and in vivo cell-based therapies have shown their potential to be used in the repair of damaged T/L. Cells can be delivered using a scaffold or alone via injection, and both strategies could be used in combination with surgical intervention for the treatment of chronic and acute injuries. However, several questions regarding suitable delivery systems, cell concentration, the best cell source and optimized expansion protocols must be answered before an efficient and safe cell-based therapy for $\mathrm{T} / \mathrm{L}$ repair can be established. 


\subsection{Conclusions and future directions}

At present, surgical intervention remains the gold standard of treatment in cases of $\mathrm{T} / \mathrm{L}$ rupture. However, as mentioned above, this strategy is not always sufficient to restore the biomechanical properties of the damaged T/L. Several studies have recently been performed exploring alternative strategies such as the use of GFs, cells and biomaterials in order to promote the repair and healing of these tissues. These strategies could be combined with the standard surgical reconstruction procedure in order to promote healing and enhance graft integration. This would improve the mechanical properties of the $\mathrm{T} / \mathrm{L}$ and allow the patient a faster return to normality. However, several issues remain unanswered regarding these strategies, such as their efficacy, safety, translation to the clinic and the variability of the published results. In the short term, the use of autografts for ACL reconstruction will continue to be the first choice; however, the foundations of tissue engineering are likely to lead to the creation of in vitro functional $T / L$ to be used in the treatment of musculoskeletal injuries through a combination of cells, biomaterials, GF and mechanical stimulus. 


\subsection{References}

1. James R, et al., Tendon: biology, biomechanics, repair, growth factors, and evolving treatment options. J Hand Surg Am, 2008. 33(1): p. 102-12.

2. Griffin LY, et al., Noncontact anterior cruciate ligament injuries: risk factors and prevention strategies. J Am Acad Orthop Surg, 2000. 8(3): p. 141-50.

3. Chen L, et al., Medial collateral ligament injuries of the knee: current treatment concepts. Curr Rev Musculoskelet Med, 2008. 1(2): p. 108-113.

4. Medicine, J.H. Knee Ligament Repair. [cited 2016 August]; Available from: http://www.hopkinsmedicine.org/healthlibrary/test_procedures/orthopaedic/knee_liga ment repair_92,p07675/.

5. Baumhauer JF and O'Brien T, Surgical Considerations in the Treatment of Ankle Instability. J Athl Train, 2002. 37(4): p. 458-462.

6. Krans, B. ACL Reconstruction. 2016 [cited 2016 August]; Available from: http://www.healthline.com/health/acl-reconstruction\#Overview1.

7. Voleti PB, Buckley MR, and Soslowsky LJ, Tendon healing: repair and regeneration. Annu Rev Biomed Eng, 2012. 14: p. 47-71.

8. Nourissat G, Berenbaum F, and Duprez D, Tendon injury: from biology to tendon repair. Nat Rev Rheumatol, 2015. 11(4): p. 223-33.

9. Yang G, Rothrauff BB, and Tuan RS, Tendon and ligament regeneration and repair: clinical relevance and developmental paradigm. Birth Defects Res C Embryo Today, 2013. 99(3): p. 203-22.

10. CB, F., Ligament structure, physiology and function. J Musculoskelet Neuronal Interact, 2004. 4(2): p. 199-201.

11. Rumian AP, Wallace AL, and Birch HL, Tendons and ligaments are anatomically distinct but overlap in molecular and morphological features--a comparative study in an ovine model. J Orthop Res, 2007. 25(4): p. 458-64.

12. Wenstrup RJ, et al., Type $V$ collagen controls the initiation of collagen fibril assembly. J Biol Chem, 2004. 279(51): p. 53331-7.

13. Mithieux SM and Weiss AS, Elastin. Adv Protein Chem, 2005. 70(437-61).

14. Ilic MZ, et al., Proteoglycans and catabolic products of proteoglycans present in ligament. Biochem J, 2005. 385(2): p. 381-8.

15. Juneja SC and V. C., Defects in tendon, ligament, and enthesis in response to genetic alterations in key proteoglycans and glycoproteins: a review. Arthritis, 2013(154812).

16. Ansorge $\mathrm{HL}$, Beredjiklian PK, and Soslowsky LJ, CD44 deficiency improves healing tendon mechanics and increases matrix and cytokine expression in a mouse patellar tendon injury model. J Orthop Res, 2009. 27(10): p. 1386-91.

17. Docheva D, et al., Tenomodulin is necessary for tenocyte proliferation and tendon maturation. Mol Cell Biol, 2005. 25(699-705).

18. Hodgson RJ, O'Connor PJ, and Grainger AJ, Tendon and ligament imaging. Br J Radiol, 2012. 85(1016): p. 1157-72.

19. Amiel D, et al., Tendons and ligaments: a morphological and biochemical comparison. J Orthop Res, 1984. 1(3): p. 257-65.

20. Kannus P, Structure of the tendon connective tissue. Scand J Med Sci Sports, 2000. 10(6): p. 312-20. 
21. N, G., et al., Tendon structure and extracellular matrix components are affected by spasticity in cerebral palsy patients. Muscles Ligaments Tendons J., 2013. 3(1): p. 42-50.

22. $\mathrm{Xu} Y$ and Murrell GA, The basic science of tendinopathy. Clin Orthop Relat Res, 2008. 466(7): p. 1528-38.

23. Gross G and Hoffmann A, Therapeutic Strategies for Tendon Healing Based on Novel Biomaterials, Factors and Cells. Pathobiology, 2013. 80(4): p. 203-10.

24. Childress MA and Beutler A, Management of chronic tendon injuries. Am Fam Physician, 2013. 87(7): p. 486-90.

25. Ortholnfo. Sprains, Strains and Other Soft-Tissue Injuries. 2015 [cited 2016 August]; Available from: http://orthoinfo.aaos.org/topic.cfm?topic=A00111.

26. Woo SL, et al., Biomechanics of knee ligaments: injury, healing, and repair. J Biomech, 2006. 39(1): p. 1-20.

27. YF, G., Ligament Injury and Repair: Current Concepts. HKPJ, 2002. 20(1): p. 22-29.

28. Kiapour AM and Murray MM, Basic science of anterior cruciate ligament injury and repair. Bone Joint Res, 2014.3(2): p. 20-31.

29. Molloy T, Wang Y, and Murrell G, The roles of growth factors in tendon and ligament healing. Sports Med, 2003. 33(5): p. 381-94.

30. Sharma P and Maffulli N, Biology of tendon injury: healing, modeling and remodeling. J Musculoskelet Neuronal Interact, 2006. 6(2): p. 181-90.

31. Marx RE, et al., Platelet-rich plasma: Growth factor enhancement for bone grafts. Oral Surg Oral Med Oral Pathol Oral Radiol Endod, 1998. 85(6): p. 638-46.

32. Chen $\mathrm{CH}$, et al., Tendon healing in vivo: gene expression and production of multiple growth factors in early tendon healing period. J Hand Surg Am, 2008. 33(10): p. 1834-42.

33. Natsu-ume T, et al., Temporal and spatial expression of transforming growth factor-beta in the healing patellar ligament of the rat. J Orthop Res, 1997. 15(6): p. 837-43.

34. Yuan T, Z. CQ, and W. JHC, Augmenting tendon and ligament repair with platelet-rich plasma (PRP). Muscles Ligaments Tendons J, 2013. 3(3): p. 139-149.

35. Kashiwagi K, et al., Effects of transforming growth factor-beta 1 on the early stages of healing of the Achilles tendon in a rat model. Scand J Plast Reconstr Surg Hand Surg, 2004. 38(4): p. 193-7.

36. Abrahamsson SO, Lundborg G, and Lohmander LS, Recombinant human insulin-like growth factor-I stimulates in vitro matrix synthesis and cell proliferation in rabbit flexor tendon. J Orthop Res, 1991. 9(4): p. 495-502.

37. Abrahamsson SO and Lohmander S, Differential effects of insulin-like growth factor-I on matrix and DNA synthesis in various regions and types of rabbit tendons. J Orthop Res, 1996. 14(3): p. 370-6.

38. Khan U, Edwards JC, and McGrouther DA, Patterns of cellular activation after tendon injury. J Hand Surg Br, 1996. 21(6): p. 813-60.

39. Chan BP, et al., Effect of basic fibroblast growth factor. An in vitro study of tendon healing. Clin Orthop Relat Res, 1997(342): p. 239-47.

40. Chan BP, et al., Effects of basic fibroblast growth factor (bFGF) on early stages of tendon healing: a rat patellar tendon model. Acta Orthop Scand, 2000. 71(5): p. 513-8.

41. Takahashi $S$, et al., Effect of recombinant basic fibroblast growth factor (bFGF) on fibroblast-like cells from human rotator cuff tendon. Tohoku J Exp Med, 2002. 198(4): p. 207-14. 
42. LE, S., Effects of serum, fibroblast growth factor, and platelet-derived growth factor on explants of rat tail tendon: a morphological study. Acta Anat (Basel). 1985. 123(4): p. 24752.

43. Yoshikawa $Y$ and A. SO, Dose-related cellular effects of platelet-derived growth factor-BB differ in various types of rabbit tendons in vitro. Acta Orthop Scand, 2001. 72(3): p. 287-92.

44. Spindler KP, Nanney LB, and D. JM, Proliferative responses to platelet-derived growth factor in young and old rat patellar tendon. Connect Tissue Res, 1995. 31(2): p. 171-7.

45. Jackson JR, et al., Expression of vascular endothelial growth factor in synovial fibroblasts is induced by hypoxia and interleukin 1beta. J Rheumatol, 1997. 24(7): p. 1253-9.

46. Boyer MI, et al., Quantitative variation in vascular endothelial growth factor mRNA expression during early flexor tendon healing: an investigation in a canine model. J Orthop Res, 2001. 19(5): p. 869-72.

47. Petersen $\mathrm{W}$, et al., The angiogenic peptide vascular endothelial growth factor (VEGF) is expressed during the remodeling of free tendon grafts in sheep. Arch Orthop Trauma Surg, 2003. 123(4): p. 168-74.

48. Rickert M1, et al., A growth and differentiation factor-5 (GDF-5)-coated suture stimulates tendon healing in an Achilles tendon model in rats. Growth Factors, 2001. 19(2): p. 115-26.

49. Wolfman NM, et al., Ectopic induction of tendon and ligament in rats by growth and differentiation factors 5, 6, and 7, members of the TGF-beta gene family. J Clin Invest, 1997. 100(2): p. 321-30.

50. Bunker DLJ, et al., Tendon to bone healing and its implications for surgery. Muscles Ligaments Tendons J, 2014. 4(3): p. 343-350.

51. Lu HH and J. J, Interface tissue engineering and the formulation of multiple-tissue systems. Adv Biochem Eng Biotechnol, 2006. 102: p. 91-111.

52. Oryan A, Moshiri A, and Meimandi-Parizi A, Graft Selection in ACL Reconstructive Surgery. Curr Orthop Pract, 2013. 24(3): p. 321-333.

53. Lohmander LS, et al., The long-term consequence of anterior cruciate ligament and meniscus injuries: osteoarthritis. Am J Sports Med, 2007. 35(10): p. 1756-69.

54. Clinic, T.Y.K. ACL Injuries. [cited 2016 August]; Available from: http://yorkshirekneeclinic.com/knee-conditions/acl-injuries/.

55. Goh JC, et al., Tissue-engineering approach to the repair and regeneration of tendons and ligaments. Tissue Eng Part A, 2003. 9(1): p. S31-44.

56. Font Tellado S, Balmayor ER, and Van Griensven $M$, Strategies to engineer tendon/ligament-to-bone interface: Biomaterials, cells and growth factors. Adv Drug Deliv Rev, 2015. 94: p. 126-40.

57. Spindler KP, et al., Patellar tendon and anterior cruciate ligament have different mitogenic responses to platelet-derived growth factor and transforming growth factor beta. J Orthop Res, 1996. 14(4): p. 542-6.

58. Batten ML, Hansen JC, and D. LE, Influence of dosage and timing of application of plateletderived growth factor on early healing of the rat medial collateral ligament. J Orthop Res, 1196. 14(5): p. 736-41.

59. Hildebrand KA, et al., The effects of platelet-derived growth factor-BB on healing of the rabbit medial collateral ligament. An in vivo study. Am J Sports Med, 1998. 26(4): p. 54954.

60. Letson AK and Dahners LE, The effect of combinations of growth factors on ligament healing. Clin Orthop Relat Res, 1994(308): p. 207-12. 
61. Kobayashi D, et al., Effect of basic fibroblast growth factor on the healing of defects in the canine anterior cruciate ligament. Knee Surg Sports Traumatol Arthrosc, 1997. 5(3): p. 18994.

62. Nau T and Teuschl A, Regeneration of the anterior cruciate ligament: Current strategies in tissue engineering. World J Orthop, 2015. 6(1): p. 127-36.

63. Mascarenhas R and MacDonald PB, Anterior cruciate ligament reconstruction: $a$ look at prosthetics - past, present and possible future. Mcgill J Med, 2008. 2008(11): p. 1.

64. Barry M, et al., Histological changes associated with an artificial anterior cruciate ligament. J Clin Pathol, 1995. 48(6): p. 556-9.

65. López-Vázquez E, et al., Reconstruction of the anterior cruciate ligament with a Dacron prosthesis. J Bone Joint Surg Am, 1991. 73(9): p. 1294-300.

66. McPherson GK, et al., Experimental mechanical and histologic evaluation of the Kennedy ligament augmentation device. Clin Orthop Relat Res, 1985(196): p. 186-95.

67. Dunn MG, et al., Development of fibroblast-seeded ligament analogs for $A C L$ reconstruction. J Biomed Mater Res A, 1995. 29(11): p. 1363-71.

68. Bellincampi LD, et al., Viability of fibroblast-seeded ligament analogs after autogenous implantation. J Orthop Res, 1998. 16(4): p. 414-20.

69. Panas-Perez E, Gatt CJ, and D. MG, Development of a silk and collagen fiber scaffold for anterior cruciate ligament reconstruction. J Mater Sci Mater Med, 2013. 24(1): p. 257-65.

70. Fanggang B, et al., Anterior Cruciate Ligament Reconstruction in a Rabbit Model Using SilkCollagen Scaffold and Comparison with Autograft. PLoS One, 2015. 10(5).

71. Ho JO, Sawadkar P, and Mudera V, A review on the use of cell therapy in the treatment of tendon disease and injuries. J Tissue Eng, 2014. 5.

72. Petrigliano FA, McAllister DR, and Wu BM, Tissue engineering for anterior cruciate ligament reconstruction: a review of current strategies. Arthroscopy, 2006. 22(4): p. 44151.

73. Leong NL, Petrigliano FA, and McAllister DR, Current tissue engineering strategies in anterior cruciate ligament reconstruction. J Biomed Mater Res A, 2014. 102(5): p. 1614-24.

74. Liu W, et al., Repair of tendon defect with dermal fibroblast engineered tendon in a porcine mode. Tissue Eng Part A, 2006. 12(4): p. 775-88.

75. Wang A, et al., Evidence for the Durability of Autologous Tenocyte Injection for Treatment of Chronic Resistant Lateral Epicondylitis: Mean 4.5-Year Clinical Follow-up. Am J Sports Med, 2015. 43(7): p. 1775-83.

76. Clarke AW, et al., Skin-derived tenocyte-like cells for the treatment of patellar tendinopathy. Am J Sports Med, 2011. 39(3): p. 614-23.

77. PP, L., Stem cell technology for tendon regeneration: current status, challenges, and future research directions. Stem Cells Cloning, 2015. 8: p. 163-74.

78. Yao L, et al., Phenotypic drift in human tenocyte culture. Tissue Eng, 2006. 12(7): p. 18439.

79. Schwarz R, Colarusso L, and Doty P, Maintenance of differentiation in primary cultures of avian tendon cells. Exp Cell Res, 1976. 102(1): p. 63-71.

80. Jelinsky SA, et al., Tendon-selective genes identified from rat and human musculoskeletal tissues. J Orthop Res, 2010. 28(3): p. 289-97.

81. Ge Z, Goh JC, and L. EH, Selection of cell source for ligament tissue engineering. Cell Transplant, 2005. 14(8): p. 573-83. 
82. Van Eijk F, et al., Tissue engineering of ligaments: a comparison of bone marrow stromal cells, anterior cruciate ligament, and skin fibroblasts as cell source. Tissue Eng, 2004. 10(56): p. 893-903.

83. Liu $\mathrm{H}$, et al., A comparison of rabbit mesenchymal stem cells and anterior cruciate ligament fibroblasts responses on combined silk scaffolds. Biomaterials, 2008. 29(10): p. 1443-53.

84. Hankemeier $\mathrm{S}$, et al., Bone marrow stromal cells in a liquid fibrin matrix improve the healing process of patellar tendon window defects. Tissue Eng Part A, 2009. 15(5): p. 1019-30.

85. Ouyang HW, et al., Knitted poly-lactide-co-glycolide scaffold loaded with bone marrow stromal cells in repair and regeneration of rabbit Achilles tendon. Tissue Eng, 2003. 2003(9): p. 3.

86. Juncosa-Melvin $\mathrm{N}$, et al., The effect of autologous mesenchymal stem cells on the biomechanics and histology of gel-collagen sponge constructs used for rabbit patellar tendon repair. Tissue Eng, 2006. 12(2): p. 369-79.

87. Kryger GS, et al., A comparison of tenocytes and mesenchymal stem cells for use in flexor tendon tissue engineering. J Hand Surg Am, 2007. 32(5): p. 597-605.

88. Awad HA, et al., Repair of patellar tendon injuries using a cell-collagen composite J Orthop Res, 2003. 21: p. 420-431.

89. Harris MT, et al., Mesenchymal stem cells used for rabbit tendon repair can form ectopic bone and express alkaline phosphatase activity in constructs. J Orthop Res, 2004. 22(5): p. 998-1003.

90. Tasso R, et al., Development of sarcomas in mice implanted with mesenchymal stem cells seeded onto bioscaffolds. Carcinogenesis, 2009. 30(1): p. 150-7. 


\section{Chapter 3}

\section{TGF- $\beta 1$ activation in hamstring cells through growth factor-binding peptides on polymers}

The administration of soluble growth factors (GFs) to injured tendons and ligaments ( $T / L$ ) is known to promote and enhance the healing process. However, the administration of GFs is a complex, expensive and heavily-regulated process and only achieved by employing supraphysiological GF concentrations. In addition, for proper healing, specific and spatial immobilization of the GFs (s) is critical. We hypothesized that biomaterials functionalized with GFs-binding peptides can be employed to capture endogenous GFs in a spatiallycontrolled manner, thus overcoming the need for the exogenous administration of supraphysiological doses of GFs. Here we demonstrate that the modification of films of polycaprolactone ( $P C L$ ) with transforming growth factor $B_{1}$ (TGF-B1)-binding peptides allows GFs to be captured and presented to the target cells. Moreover, using a TGF- $B$ reporter cell line and immunocytochemistry, we show that the GFs retained their biological activity. In human primary tendon cells, the immobilized TGF- 81 activated TGF- 8 target genes ultimately lead to a 2.5 -fold increase in collagen matrix production. In vivo implantation in rats clearly shows an accumulation of TGF- 61 on the polymer films functionalized with the TGF-81-binding peptide when compared with the native films. This accumulation leads to an increase in the recruitment of inflammatory cells at day 3 and an increase in the fibrogenic response and vascularization around the implant at day 7. The results herein presented will endow current and future medical devices with novel biological properties and by doing so will accelerate $T / L$ healing. 


\subsection{Introduction}

The anterior cruciate ligament $(A C L)$ is one of the most commonly-injured ligaments in the knee, with approximately 200,000 incidences of injury annually in the United States alone [1]. Due to its low self-regenerative capacity, surgical intervention is often needed in order to re-establish the biomechanical properties of the injured tissue. Auto- or allografts are used for ACL reconstruction; however, donor-site morbidity, pain, graft failure and risk of disease transmission are common problems associated with the procedure [2]. Moreover, in most cases, the biomechanical properties of the grafted tissue do not match the ACL's original properties, leading to maladaptive joint issues. Recent years have shown an increase in the number of publications exploring the potential of administrating GFs to promote tissue healing; therefore, the potential of GF administration to promote and enhance the healing process of damaged $A C L$ is a realistic opportunity for improving the clinical outcome. GFs are key players in the wound healing cascade, orchestrating, in a temporal and spatial manner, cellular mechanisms crucial for the proper healing of the tissue, such as cell signalling, proliferation, migration, survival and differentiation [3, 4]. The easiest and simplest mechanism to deliver GFs is in a soluble form. It has been shown in canines that the exogenous administration of transforming beta 1 (TGF- $\beta 1$ ) significantly increases the bonding strength of the graft that was used to replace the original ACL [5]. In a rabbit model of patellar tendon injury, it was shown that the administration of TGF- $\beta 1$ directly following wound closure increases the tangent modulus and the tensile strength of the regenerated fibrous tissue [6]. However, due to the low stability of GFs in the body and diffusion-related problems, supraphysiological concentrations and systematic administration are required in order to achieve the desired effect [4]. In order to overcome these problems, GFs have been immobilized onto biomaterials in order to avoid diffusion-related issues and achieve colocalization of the GFs in the wounded tissue. GFs can be immobilized in a covalent and non-covalent manner, via direct electrostatic means or by reactions between the GF and matrices, or interactions via other biological molecules such as heparin, gelatin or fibronectin [3, 7]. However, few studies have explored this strategy for the enhancement of T/L healing. Sahoo et al. (2010) showed that the incorporation of basic fibroblast growth factor (bFGF) in poly(lactic-co-glycolic acid) (PLGA) nanofibrous scaffolds induces proliferation of rabbit bone marrow stromal cells (BMSC), upregulates the expression of $\mathrm{T} / \mathrm{L}$-related genes such as type I and III collagen as well as the production of collagen and tenascin-C, and induces differentiation in T/L-like fibroblasts [8]. Kimura et al. (2008) reported the combination of poly-L-lactide acid (PLLA) and a gelatin 
hydrogel incorporating bFGF for the regeneration of rabbit ACLs. The results of this study showed that the controlled release of $\mathrm{bFGF}$ promotes the regeneration of the ligamentbone interface, ultimately resulting in an increase in the mechanical strength of the healed ACL compared to the control groups [9]. In both studies, bFGF was physically entrapped in the material, which is usually associated with an initial burst release of the GFs and therefore the requirement of high concentrations of GF in order to achieve longterm effects. The immobilization of GFs through the formation of a covalent bond with the biomaterial or non-covalent interactions with affinity molecules (e.g., heparin) can achieve a sustained release of the GFs. Nevertheless, the formation of this covalent bond often hinders the bioactivity of the GF, mostly due to conformational changes, ultimately affecting its biological activity [3]. Additionally, permanent immobilization of the GFs through a covalent bond will present the factor to the cells in a non-natural immobile way. In contrast, the non-covalent presentation of GFs is a more natural way to deliver these to the injured tissue. However, heparin-like structures do not offer any specificity towards the GFs and consequently other circulating GFs could be immobilized by these structures. It has previously been shown that supramolecular nanofibers functionalized with a TGF- $\beta 1$-binding peptide promote cartilage regeneration when compared with the non-functionalized nanofibers [10]. The use of short peptides to deliver GFs has several advantages over other GF delivery strategies, such as their easy and rapid synthesis using standard chemical peptide synthesis and purification using standard chromatography methods. GF-binding peptides overcome the disadvantages related to the previouslymentioned delivery strategies since the affinity towards the GFs is based on selective non-covalent interactions. We hypothesized that functionalization of biomaterials with GF-binding peptides could capture the endogenous GFs, leading to their accumulation and consequently enhancing the healing process without needing to administer exogenous GFs. Here we present for the first time a non-covalent approach to specifically immobilize TGF- $\beta 1$ (hTGF- $\beta 1$ ) on polymer films and deliver hTGF- $\beta 1$ to humanderived hamstring cells and thus promote $\mathrm{T} / \mathrm{L}$ healing. By functionalizing PCL films with a TGF- $\beta 1$-binding peptide, we were able to specifically immobilize hTGF- $\beta 1$. Subsequently, we show in vitro that the functionalized films lead to an upregulation of ECM-related genes such as collagen type I and III, culminating in the enhanced production of collagen by human hamstring-derived cells. When implanted subcutaneously in rats, the films functionalized with TGF- $\beta 1$-binding peptide capture more endogenous TGF- $\beta 1$ than the control films, ultimately contributing to enhance the fibrogenic response and vascularization around the implant. These results demonstrate the potential of using this synthetic peptide sequence to capture and accumulate native TGF- $\beta 1$ onto biomaterials 


\section{Chapter 3}

TGF- $\beta 1$ activation in hamstring cells through growth factor-binding peptides on polymers

in order to promote the healing of damaged tissues without the needed to administer exogenous GFs.

\subsection{Materials and methods}

\subsubsection{Materials}

$\mathrm{N}, \mathrm{N}, \mathrm{N}, \mathrm{N}^{\prime}$-Tetramethyl-O-(1H-benzotriazol-1-yl)uronium hexafluorophosphate (HBTU) was obtained from MultiSynTech. Chloroform and 1-methyl-2-pyrrolidinone (NMP) were purchased from WR Chemicals. $\mathrm{NaOH}$ was obtained from Riedel-de Haën. All other reagents or products were purchased from Sigma-Aldrich unless noted otherwise.

\subsubsection{Peptide synthesis and purification}

The synthesis of the peptide sequences was performed using standard Fmoc-solid phase peptide synthesis in a Syro II MultiSynTech automated peptide synthesizer. The TGF- $\beta 1$ binding and nonbinding peptides with sequences KGLPLGNSH and KGHNLGLPS, respectively, were prepared on Fmoc-Rink 4-methylbenzhydrylamine (MBHA) resin (MultiSynTech $\mathrm{GmBH}, 50 \mathrm{mg}$ scale, substitution $0.52 \mathrm{mmol} / \mathrm{g}$ ), using $0.26 \mathrm{M} \mathrm{HBTU}, 0.52$ $\mathrm{M}$ of $\mathrm{N}, \mathrm{N}$-diisopropylethylamine (DIPEA), $2 \mathrm{M}$ of piperidine and $0.29 \mathrm{M}$ of each amino acid. The $\mathrm{N}$-termini of the final peptide sequences were manually acetylated in $16 \%$ acetic anhydride, 30\% DIPEA and 54\% NMP for one hour at room temperature. The peptides were cleaved from the resin and amino acid side groups were deprotected using $95 \%$ trifluoroacetic acid, $2.5 \%$ triisopropylsilane and $2.5 \%$ milliQ water. The peptides were then collected by precipitation in cold diethyl ether and the organic solvents were removed in a rotatory evaporator. The peptides were redissolved in milliQ water and lyophilized overnight. The resulting products were purified using standard preparative HPLC methods. MS (ESI): $\mathrm{m} / \mathrm{z}=964.1[\mathrm{M}+\mathrm{H}]$ (calculated 963.1 for $\mathrm{C}_{42} \mathrm{H}_{70} \mathrm{~N}_{14} \mathrm{O}_{12}$ ) for KGLPLGNSH. MS (ESI): $\mathrm{m} / \mathrm{z}=964.6[\mathrm{M}+\mathrm{H}]$ (calculated 963.1 for $\mathrm{C}_{42} \mathrm{H}_{70} \mathrm{~N}_{14} \mathrm{O}_{12}$ ) for KGHNLGLPS.

\subsubsection{Preparation of peptide-displaying PCL films}

A $12.5 \%(w / v)$ solution of $P C L$ in chloroform was prepared and homogenized by sonication. When the solution was completely homogeneous, $\mathrm{PCL}$ films were prepared by casting in a petri dish, pre-silanized with a PFDTS $(1 \mathrm{H}, 1 \mathrm{H}, 2 \mathrm{H}, 2 \mathrm{H}$ perfluorodecyltrichlorosilane, $\geq 97 \%, A B C R \mathrm{GmbH}$ ) anti-sticky layer. Upon solvent evaporation, the polymer was melted and allowed to again solidify. The polymer was then cut in circular films with a diameter of $21 \mathrm{~mm}$ in order to fit inside the wells of a 12- 
well plate. The individual circular films were extensively washed with demi-water and milliQ water and dried with a $\mathrm{N}_{2}$ stream. The dried films were exposed to oxygen plasma for $5 \mathrm{~min}$ (at an oxygen pressure of $1.0 \mathrm{bar}$, a vacuum pressure of $200 \mathrm{mbar}$ and a current of $40 \mathrm{~A}$ ) and subsequently immersed in a $1 \mathrm{M} \mathrm{NaOH}$ solution for one hour with gentle agitation. PCL films were then washed and dried as mentioned above, and incubated with a solution of $50 \mathrm{mM}$ 1:1 NHS/EDC in MES buffer for one hour with agitation. PCL films were washed and dried again as mentioned above and were incubated with $1 \mathrm{mM}$ of the peptide in phosphate buffered saline (PBS) for 4 hours with agitation. Films were then washed extensively with PBS and sterilized by incubating the films overnight in a solution of $10 \%$ penicillin/streptomycin (Life Technologies) in PBS prior to cell seeding.

\subsubsection{Water contact angle measurements}

The wettability of the PCL films was determined by a drop contact angle system (Krüss Contact Angle Measuring System G10). The contact angle was measured and calculated using Drop Analysis software. All reported contact angles are the average of $n=6$ measurements. MilliQ water was used to measure the contact angle of the films.

\subsubsection{TGF- $\beta 1$ binding and immunofluorescence}

The PCL films were incubated with $1 \mu \mathrm{g} / \mathrm{mL}$ of hTGF- $\beta 1$ (PeproTech) in $4 \mathrm{mM}$ hydrochloric acid $(\mathrm{HCl})$ containing $1 \mathrm{mg} / \mathrm{mL}$ bovine serum albumin (BSA) for one hour with gentle agitation. The films were then washed for $10 \mathrm{~min}$ three times with $1 \mathrm{mM}$ phosphate buffered saline tween-20 (PBST) and then with PBS alone for a further $10 \mathrm{~min}$. Next, the films were blocked for one hour with PBS containing $1 \%(w / v)$ BSA and subsequently washed as described above. Afterwards, the films were incubated with a $5 \mu \mathrm{g} / \mathrm{mL}$ solution of the primary antibody (mouse monoclonal anti-human TGF- $\beta 1, R \& D$ systems) in blocking solution for one hour with agitation. The films were washed as mentioned above and then incubated with a $4 \mu \mathrm{g} / \mathrm{mL}$ solution of the secondary antibody (goat antimouse Alexa Fluor 546, Invitrogen) in PBS containing 1\% w/v BSA for one hour with gentle agitation. Prior to fluorescence microscopy, the films were washed for 10 min three times with $1 \mathrm{mM}$ PBST, rinsed three times with PBS and dried under a $\mathrm{N}_{2}$ stream. For cell experiments, the sterile films were washed three times with PBS and incubated with hTGF- $\beta 1$ in sterile $4 \mathrm{mM} \mathrm{HCl}$ containing $1 \mathrm{mg} / \mathrm{mL} \mathrm{BSA}$ for one hour with gentle agitation. Subsequently, the films were extensively washed with PBST and PBS to remove any traces of the washing buffer prior to cell seeding. 


\section{Chapter 3}

\subsubsection{In vitro quantification of bound TGF- $\beta 1$}

Bound hTGF- $\beta 1$ was quantified by incubating the films with $1 \mu \mathrm{g} / \mathrm{mL}$ of TGF- $\beta 1$ in $4 \mathrm{mM} \mathrm{HCl}$ containing $1 \mathrm{mg} / \mathrm{mL}$ BSA for one hour with gentle agitation. The supernatant was collected and the films were washed for 30 min with PBST $(0.1 \%(v / v))$. The buffer was then collected and mixed with the previously-collected supernatant. The collected solutions were analysed for unbound hTGF- $\beta 1$ by an anti-human TGF- $\beta 1$ ELISA kit (Abcam $A B 100647)$, according to the manufacturer's instructions. The amount of immobilized hTGF- $\beta 1$ was calculated based on the difference between the incubation solution and the unbound hTGF- $\beta 1$ quantified by the ELISA kit.

\subsubsection{Cell culture}

Mink Lung Epithelium Cells (MLEC - a kind gift from Daniel's Rifkin lab) were expanded in Dulbecco's Modified Eagle Medium (DMEM, Life Technologies, Gaithersburg, MD) supplemented with 10\% Fetal Bovine Serum (FBS, Life Technologies), $100 \mathrm{U} / \mathrm{mL}$ penicillin (Life Technologies), $100 \mu \mathrm{g} / \mathrm{mL}$ streptomycin (Life Technologies) and $2 \mathrm{mM}$ L-glutamine (Life Technologies). Cells were grown at $37^{\circ} \mathrm{C}$ in a humid atmosphere with $5 \% \mathrm{CO}_{2}$. The medium was refreshed twice per week and cells were used for further sub-culturing or cryopreservation on reaching near confluence. This cell line, initially described by Rifkin et al. (1994), expresses luciferase under the control of a TGF target gene (Plasminogen activator inhibitor 1 - PAI-1 promoter) [11].

Hamstring cells (HT22, $\mathrm{P}_{3}$-4) were isolated using an outgrowth procedure, as previously described [12], and cultured in a-minimal essential medium (aMEM, Life Technologies) with $10 \%$ FBS (Gibco, Life Technologies), $100 \mathrm{U} / \mathrm{mL}$ penicillin, $100 \mu \mathrm{g} / \mathrm{ml}$ streptomycin and 0,2mM L-ascorbic acid-2-phosphate magnesium salt (ascorbic acid, Life Technologies). Cells were grown at $37^{\circ} \mathrm{C}$ in a humid atmosphere with $5 \% \mathrm{CO}_{2}$. The medium was refreshed twice per week, and cells were used for further sub-culturing or cryopreservation on reaching near confluence. Experiments with hamstring cells were performed with cells until passage 4 .

\subsubsection{Luciferase assay}

MLECs were seeded at 64,000 cells $/ \mathrm{cm}^{2}$ and allowed to attach overnight at $37^{\circ} \mathrm{C}$ in a $5 \%$ $\mathrm{CO}_{2}$ incubator. The medium was then replaced by DMEM without FBS and the cells were incubated for an additional period of 24 hours. Cells were lysed and the luciferase quantified according to the manufacturer's protocol (Promega, E4530). Luciferase 
values were normalized for DNA content quantified by CyQUANT cell proliferation assay (Invitrogen).

\subsubsection{Smad translocation assay}

To assess the cellular localization of the Smad2/3 complex, hamstring cells were seeded at 10,000 cells $/ \mathrm{cm}^{2}$ and incubated for 24 hours at $37^{\circ} \mathrm{C}$ in a $5 \% \mathrm{CO}_{2}$ incubator. Samples were washed with PBS and cells were fixed with $4 \%(w / v)$ paraformaldehyde/PBS for 15 min at room temperature. Samples were washed with PBS and incubated with a filtered solution of $0,3 \%(w / v)$ Sudan Black in $70 \%$ ethanol for 30 min with gentle agitation. Films were washed three times for 5 min with PBS and the cell membrane was permeabilized with $0.1 \%$ Triton $\mathrm{X}-100 / \mathrm{PBS}$ for $15 \mathrm{~min}$. After rinsing three times with PBS, films were blocked with a solution of $2 \%(\mathrm{w} / \mathrm{v}) \mathrm{BSA}$ in $0.1 \%$ Triton X-100 in PBS at room temperature for one hour with gentle agitation. Monoclonal mouse anti-Smad2/3 (clone 18, BD Bioscience, 1:200) was incubated overnight at $4^{\circ} \mathrm{C}$ in the blocking solution with gentle agitation. The secondary antibody goat anti-mouse Alexa Fluor 594 (DAKO, 1:200) was incubated at room temperature for one hour in the blocking solution. Nucleic acids were stained with DAPI (Life Technologies, 1:100) for $15 \mathrm{~min}$ at room temperature. Samples were washed three times for 5 min with $0.1 \%$ Triton X-100/PBS, rinsed with PBS and dried with a $\mathrm{N}_{2}$ stream before mounting.

\subsubsection{Gene expression analysis}

For gene expression analysis, hamstring cells were seeded on films at 5,000 cells $/ \mathrm{cm}^{2}$ and cultured for 3, 7 and 14 days in culturing medium. RNA was then isolated using TRIzol combined with a NucleoSpin RNA II kit (Bioke). Subsequently, $1 \mu \mathrm{g}$ of RNA was used to synthesize CDNA using the SensiFast kit (Bioline). iQ SYBR Green Supermix (Bio-Rad) was used for quantitative polymerase chain reaction ( $q P C R$ ) on a MJ Mini ${ }^{\mathrm{TM}}$ thermal cycler (Bio-Rad). Gene expression was normalized using the housekeeping gene B2M. The primer sequences used are as follows: collagen I forward: 5'-GTC ACC CAC CGA CCA AGA AAC C-3', reverse: 5'-AAG TCC AGG CTG TCC AGG GAT G-3'; collagen III forward: 5'-GCC AAC GTC CAC ACC AAA TT-3', reverse: 5'-AAC ACG CAA GGC TGT GAG ACT-3'; sox9 forward: 5'-ATC CGG TGG TCC TTC TTG TG-3', reverse: 5'-TGG GCA AGC TCT GGA GAC TTC3'; aggrecan forward: 5'-AGG CAG CGT GAT CCT TAC C-3', reverse: 5'-GGC CTC TCC AGT CTC ATT CTC-3'; B2M forward: 5'-ACA AAG TCA CAT GGT TCA CA-3', reverse: 5'-GAC TTG TCT TTC AGC AAG GA-3'. 


\subsubsection{Collagen quantification}

For the analysis of the amount of collagen produced, hamstring cells were seeded on films at 5,000 cells $/ \mathrm{cm}^{2}$ and cultured for 7 and 14 days in culture medium. Hydroxyproline quantification was used as a direct method for the determination of the collagen content in the samples. Cells were washed with PBS, lysed with $12 \mathrm{~N} \mathrm{HCl}$ and scratched from the films. The lysate was transferred to a pressure-tight Teflon-capped vial and hydrolysed at $120^{\circ} \mathrm{C}$ for three hours. After hydrolyzation, the amount of hydroxyproline was quantified using the Hydroxyproline Colorimetric Assay Kit (BioVision) according to manufacturer's instructions.

\subsubsection{Subcutaneous implantation mouse model}

All of the animal experiments performed were approved by the animal research ethics committee of the Chinese University of Hong Kong. Eight 12-week-old Sprague Dawley male rats were used in this study. The rats were anesthetized by intraperitoneal injection of $10 \%$ ketamine $/ 2 \%$ xylazine (Kethalar, $0.3 \mathrm{ml}: 0.2 \mathrm{ml}$ ); sedation was maintained by intramuscular injection of $10 \%$ ketamine (Sigma Chemical CO, St. Louis, MO). Subcutaneous implantation of PCL was performed. In brief, once the animals were anesthetized, shaved and washed, two incisions were made and native $P C L$ and $P C L$ functionalized with a TGF- $\beta 1$ binging peptide were inserted into the pockets and fixed to the fascia. The skin wound was then closed using sutures. At day 3 and day 7 post implantation, animals were sacrificed and samples were harvested. Samples from the subcutaneous rat model were harvested at day 7 post implantation and rinsed with PBS. Harvested samples were fixed with $10 \%$ buffered formalin for 10 min and further permeabilized with PBST for 15 min. Samples were then washed with PBS and blocked with $1 \%(w / v)$ BSA for 1 hour in a shaker. Next, the samples were washed three times for 10 min with PBST and incubated with a dilution of 1:100 of the primary antibody (rabbit polyclonal anti TGF- $\beta 1$, Santa Cruz Biotechnology) overnight at $4^{\circ} \mathrm{C}$. After primary antibody incubation, samples were washed with PBST and incubated with a dilution of 1:100 of the secondary antibody (goat anti rabbit IgG-PE, Santa Cruz Biotechnology) for one hour at room temperature. Samples were washed with PBST before imaging. 


\subsubsection{Histology}

At day 3 and day 7 post implantation, PCL implants and their surrounding tissues were harvested and rinsed with PBS. Harvested samples were fixed with $10 \%$ buffered formalin overnight and embedded in paraffin. Subsequently, $5 \mu \mathrm{m}$-thick paraffin sections along the transverse plane were collected. Haematoxylin and eosin (H\&E) stained sections were examined under light microscopy (Leica Microsystems, Wetzlar, Germany).

\subsubsection{Statistical analysis}

The data were analysed using a Student's paired t-test, a one-way analysis of variance followed by a Tukey's multiple comparison test $(p<0.05)$ or a two-way analysis of variance. The values represent the mean and standard deviation of three independent measurements.

\subsection{Results}

\subsubsection{Fabrication of PCL films presenting TGF- $\beta 1$-binding peptides}

In order to immobilize hTGF- $\beta 1$ on the PCL film, a previously reported TGF- $\beta 1$-binding peptide sequence LPLGNSH was synthesized and equipped with an extra lysine and a glycine at the N-termini (Figure S3.1). The extra glycine functioned as a spacer between the film and the active sequence involved in the affinity for hTGF- $\beta 1$, whereas the extra lysine provided an amine group to covalently immobilize the peptide to the film. The Nterminus of the peptide was acetylated, and consequently the covalent immobilization of the peptide to the film was solely achieved through the free amine from the lysine side group.

To covalently bind the peptide to the film, the native PCL films were initially treated with oxygen plasma to introduce carboxylic acid groups to the surface. Next, the carboxylic acids were activated with EDC/NHS treatment, leading to the formation of amine reactive esters that were used for the covalent immobilization of the TGF- $\beta 1$ binding via the free amine present in the side chain of the lysine amino acid. Surface wettability was used to keep track of the chemical modifications during the immobilization procedure (Figure 3.1). After exposing the native PCL films to oxygen plasma for $5 \mathrm{~min}$, the hydrophobicity of the surface decreased to $38 \pm 2^{\circ}(p<0.0001)$, whereas NHS/EDC treatment led to an increase of $19 \pm 2^{\circ}(p<0.0001)$. Films with the amine reactive ester were then incubated with a PBS solution alone or with $1 \mathrm{mM}$ of the TGF- $\beta 1$-binding 
peptide in PBS. As expected, when the films with the NHS-activated acids were incubated with PBS, no significant change in the water contact angle was observed. In contrast, when the films were incubated with a solution of PBS containing the TGF- $\beta 1$ binding peptide, a further increase of $11 \pm 1^{\circ}(p<0.0001)$ in the contact angle of the surface was observed, indicating that the peptide was attached to the films.

A



B

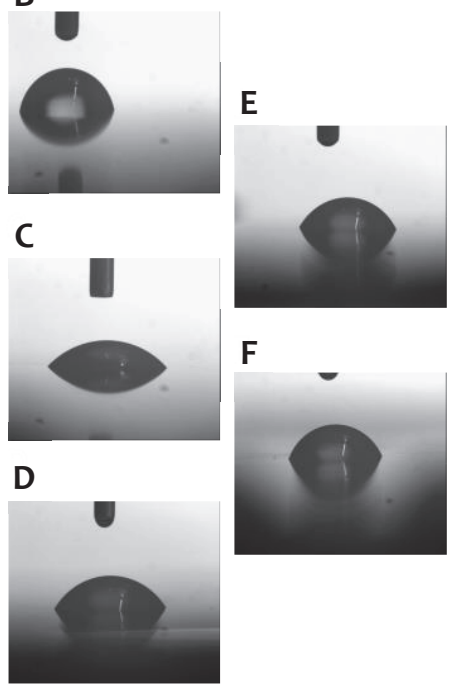

Figure 3.1. (A) Water contact angle measurements of the films during the chemical functionalization procedure. (B) 'Native film' represents the PCL films without any chemical modification; (C) 'Films after OPT' is the native PCL films exposed for 5 min to oxygen plasma; (D) 'Films Cov-NHS' indicates the films with amine reactive esters after 1 hour incubation with NHS/EDC; (E) 'PBS' is the films with reactive amine esters incubated for 4 hours with PBS; (F) 'Peptide' is the films with reactive amine esters incubated during 4 with a $1 \mathrm{mM}$ of peptide in PBS. $* * * p<0.0001$ (two-tailed unpaired $t$-test). The data represent the mean $\pm S D$ of two measurements per sample $(n=3)$.

\section{3-3.2 TGF- $\beta 1$ immobilization on the functionalized PCL films}

To confirm the attachment of the peptide to the films and the subsequent binding of the GF to the functionalized films, immunostaining was performed on both native and functionalized films incubated either with or without hTGF- $\beta$ 1. These results showed a statistically significant increase in functionalized films incubated with the hTGF- $\beta 1$ (Figure $3.2 \mathrm{~A} ; \mathrm{p}<0.0001$ ). In addition, immunostaining demonstrated a homogenous distribution of the $\mathrm{GF}$ within the film (Figure $3.2 \mathrm{~B}$ and $\mathrm{C}$ ). In addition to showing a specific interaction of hTGF- $\beta 1$ with the peptide, the absence of unspecific interactions of the primary or secondary antibody with the functionalized film was also demonstrated. When native 
$P C L$ films were incubated with hTGF- $\beta 1$, no significant increase in the fluorescence intensity was observed, indicating that no unspecific interactions occurred between hTGF- $\beta 1$ and the native films. To summarize, the fluorescence signal reported for the functionalized films is the result of the specific binding of hTGF- $\beta 1$ to the TGF- $\beta 1$-binding peptide attached to the films.

A

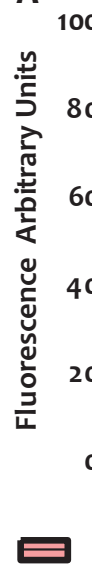



Native Film

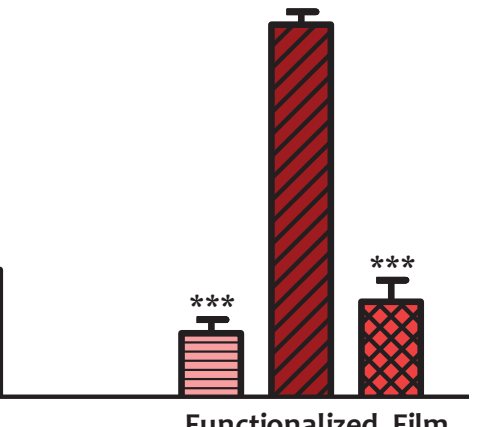

Functionalized Film

Without TGF

With TGF

\section{xex With TGF-B1 and only Secondary Antibody}

B

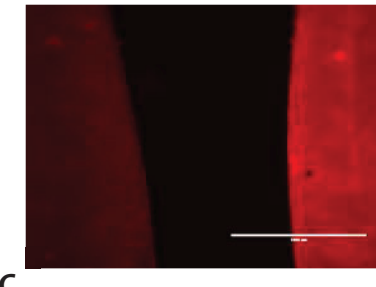

C

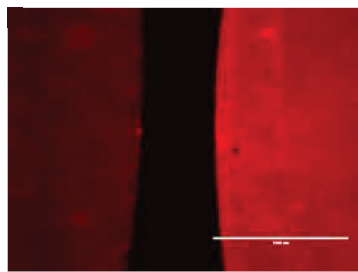

Figure 3.2. (A) Fluorescence quantification of the immunochemistry assay against hTGF- $B_{1}$ immobilized on the films. The data represent mean \pm SD of three measurements per sample $(n=2)$. $* * * p<0.0001$ (two-tailed unpaired t-test) with functionalized films incubated with hTGF-B1 and immunochemistry performed with primary and secondary antibodies. (B) Fluorescence image of the functionalized film without hTGF- 1 incubation (left) vs functionalized film with hTGF-B1 incubation (right) (bar: 1,000 $\mu \mathrm{m}$ ). (C) Fluorescence image of the native film with hTGF- 81 incubation (left) vs functionalized film with hTGF-81 incubation (right) (bar: 1,000 $\mu \mathrm{m}$ ).

In order to estimate the amount of hTGF- $\beta 1$ bound to the TGF- $\beta 1$-binding peptide functionalized film, an ELISA assay against hTGF- $\beta 1$ was performed (Figure 3.3). Our results show that no GF remained on the native film after a one-hour incubation, which is in agreement with the results from the immunostaining (Figure 3.2). In contrast, 37\% of the initial amount of hTGF- $\beta_{1}(1 \mu \mathrm{g} / \mathrm{mL})$ remained on the films functionalized with the TGF- $\beta 1$-binding peptide, giving a surface concentration of $123 \pm 16 \mathrm{ng} / \mathrm{cm}^{2}$ of hTGF- $\beta 1$ ( $p<$ 0.005). The ELISA assay further confirmed the results obtained in the immunostaining, indicating that it is possible to specifically immobilize hTGF- $\beta 1$ on the film functionalized with a TGF- $\beta 1$-binding peptide sequence. 


\section{Chapter 3}

TGF- $\beta 1$ activation in hamstring cells through growth factor-binding peptides on polymers



Figure 3.3. Quantification of the amount of immobilized hTGF-81 by the TGF-81-binding peptide was analysed using an ELISA assay against hTGF- $61 .{ }^{* *} p<0.005$ (two-tailed unpaired t-test) between native film and functionalized film. The dashed line represents the hTGF-B1 concentration of the incubation solution. The data are represented as mean $\pm S D$ of two measurements per sample $(n=3)$.

\subsubsection{Bioactivity of the immobilized TGF- $\beta 1$ in a TGF- $\beta 1$ reporter cell line}

A TGF- $\beta 1$ reporter cell line was used in order to assess whether the immobilized GF retained its bioactivity. The films were first incubated with a solution containing o or 50 ng of hTGF- $\beta 1$, the cells were seeded in the absence or presence of $10 \mu \mathrm{M}$ of a specific TGF- $\beta 1$ inhibitor (SB-431452) and the amount of luciferase was quantified and normalized to the amount of DNA (Figure 3.4).

When both native and Cov-NHS films were incubated with hTGF- $\beta 1$ before cell seeding, no increase in the luciferase activity was observed when compared with the values obtained for the same films without hTGF- $\beta 1$ incubation. However, when the films functionalized with a TGF- $\beta 1$-binding peptide were incubated with hTGF- $\beta 1$ before cell seeding, a $9 \pm 3$-fold increase in luciferase activity was observed $(p<0.01)$. In order to demonstrate that the interaction of the GF was specifically mediated by the TGF-binding peptide, a nonbinding, scrambled peptide (Figure S3.2) sequence was included as a control. When these films were incubated with hTGF- $\beta 1$ before cell seeding, an increase of $3 \pm 1$ in luciferase activity was also observed $(p<0.05)$. The inclusion of a TGF- $\beta 1$ inhibitor completely suppressed the observed luciferase activity $(p<0.05)$. 


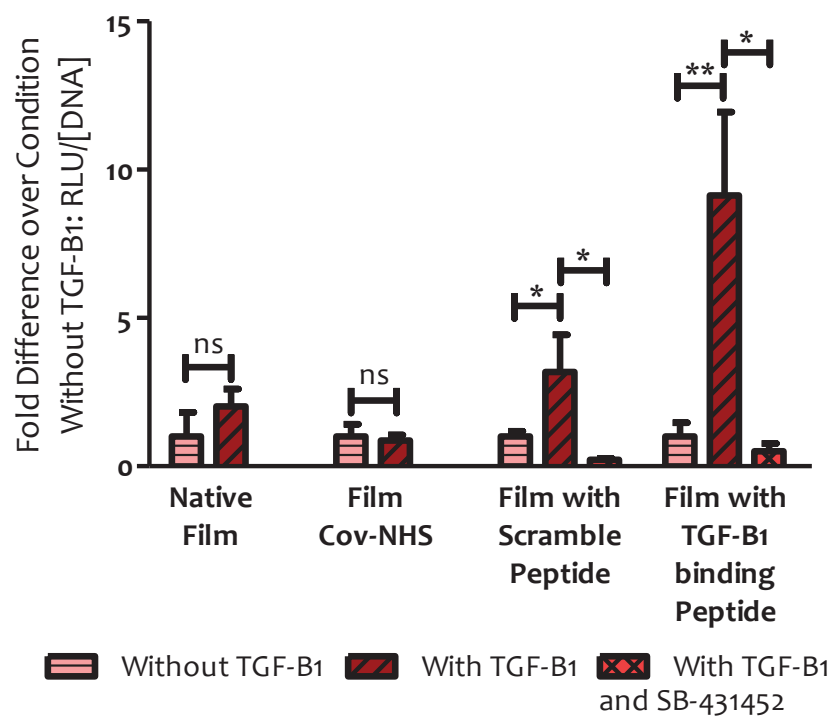

Figure 3.4. Effect of immobilized hTGF-B1 on the expression of luciferase by a TGF-8 reporter cell line. Luciferase values were corrected for the amount of DNA and normalized to the condition without incubation of hTGF- 81 prior to cell seeding. $* p<0.05$ and $* * p<0.01$ (two-tailed unpaired t-test). The data are represented as mean $\pm S D$ of two measurements per sample $(n=3)$.

3.3.4 Immobilized hTGF- $\beta 1$ activates the TGF pathway via Smad2/3 in human hamstring cells

The bioactivity of the immobilized hTGF- $\beta 1$ was evaluated in human hamstring-derived cells, which were isolated from the hamstring graft - the most commonly used graft for $A C L$ reconstruction. To that end, the intracellular localization of the SMAD2/3 complex, a key player in the TGF- $\beta 1$ signalling pathway, was monitored (Figure 3.5). Upon phosphorylation, this complex binds to SMAD4 and translocates from the cytoplasm into the nucleus, activating the transcription of TGF- $\beta 1$ target genes [13]. When the cells were seeded on functionalized films without pre-incubation with hTGF- $\beta 1$, the SMAD2/3 complex was found both in the cytoplasm and in the nucleus. In contrast, when the cells were seeded on the functionalized films that were previously incubated with hTGF- $\beta 1$, the SMAD2/3 complex was mostly detected in the nucleus, clearly demonstrating a TGFb1-mediated translocation mechanism. 

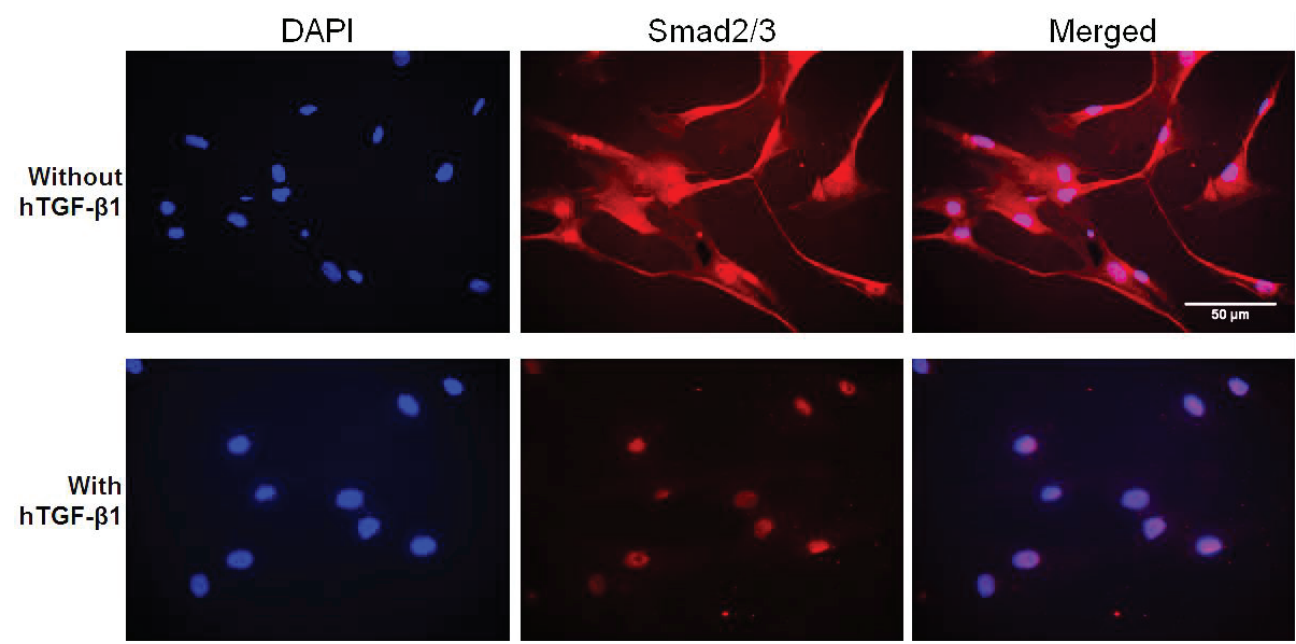

Figure 3.5. Immobilized hTGF-B1 induces the translocation of Smad2/3 into the nucleus in humanderived hamstring cells. Scale bar: $50 \mu \mathrm{m}$.

\subsubsection{Immobilized hTGF- $\beta 1$ specifically activates TGF- $\beta 1$ target genes}

In tissue healing, various components of the extracellular matrix (ECM), such as collagens and proteoglycans, are responsible for the restoration of tissue homeostasis. Given that the immobilized hTGF- $\beta 1$ promotes SMAD2/3 translocation to the nucleus, the expression of TGF- $\beta 1$ target genes in human-derived hamstring cells was evaluated. The expression of collagen types I and III, which are the major components of T/L, and aggrecan (Acan), which is a component of ECM and Sox9 and a chondrogenic differentiation marker, were studied by qPCR (Figure 3.6).

Our results show that the expression of collagen type I, Acan and Sox9 are similar over time. A statistically significant upregulation was observed at day 3 for the three genes on the functionalized films with immobilized hTGF- $\beta 1$. For later time points (days 7 and 14), a downregulation was observed in the expression of these genes for all conditions tested. In the case of collagen type III, an upregulation was observed in the case of functionalized films with immobilized hTGF- $\beta 1$, which, in contrast to the previouslymentioned genes, was maintained over time. These data show that the immobilized hTGF- $\beta 1$ is regulating the transcription of TGF- $\beta 1$-target genes, as previously reported [1417]. Interestingly, in the case of collagen type III, this activation is sustained without needing to add exogenous hTGF- $\beta 1$ to the cells. 

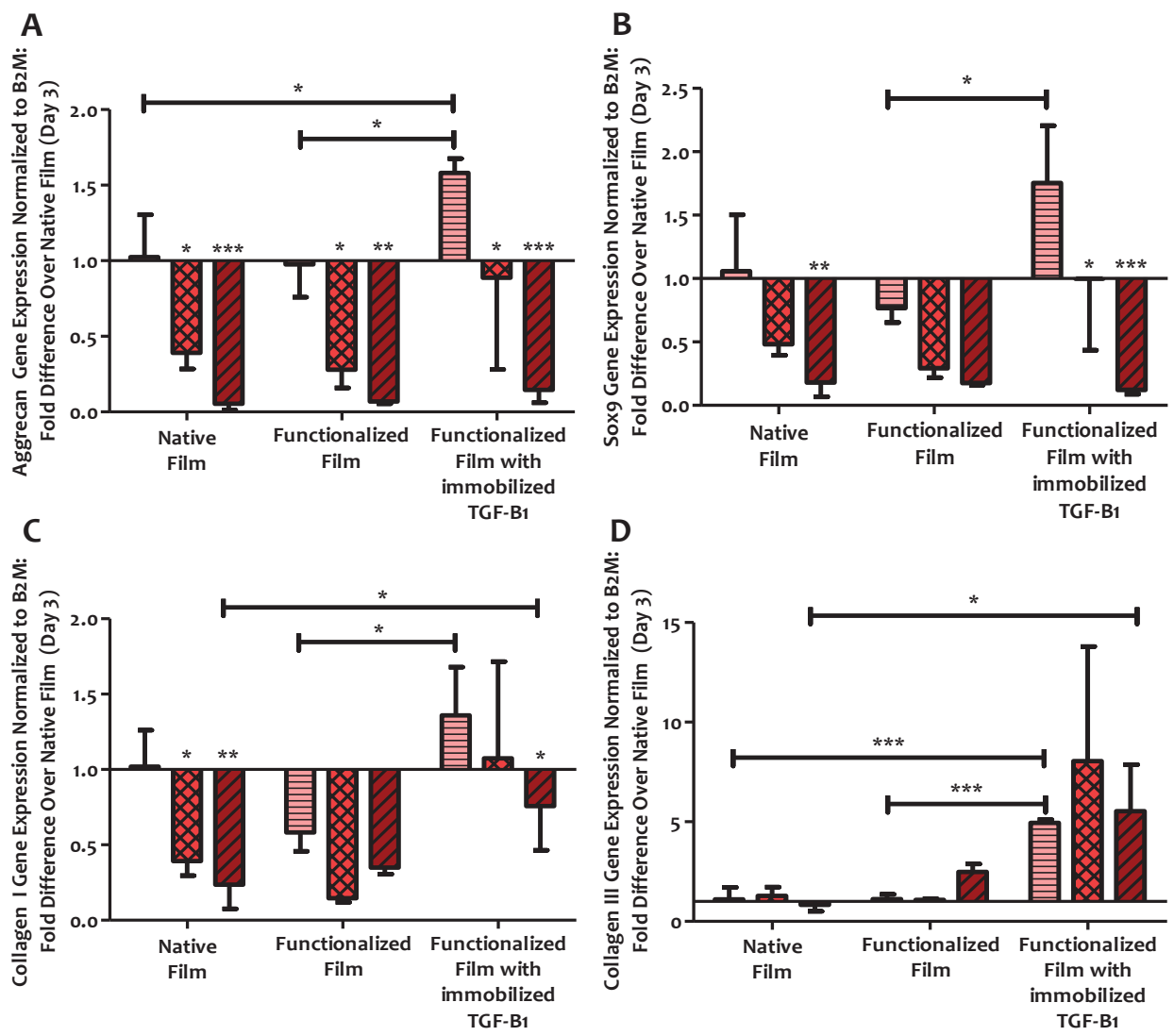

Day 3

Day 7

Day 14

Figure 3.6. Immobilized hTGF-B1 induces the expression of TGF- 81 target genes in human-derived hamstring cells. qPCR analysis of (A) aggrecan, (B) Sox9, (C) collagen type I and (D) collagen type III. * $p<0.05 ; * * p<0.01 ; * * p<0.0001$ with a two-way ANOVA for the analysis of gene expression over time and a one-way ANOVA with Tukey's post-hoc test for the analysis of gene expression between all conditions at a particular time point. The data are represented as mean \pm SD of triplicates.

\subsubsection{Effects of immobilized hTGF- $\beta 1$ on endogenous collagen production}

In order to ascertain whether the upregulation observed for collagen at the gene level was effectively leading to protein upregulation, the total amount of hydroxyproline was quantified as a direct measure of collagen protein (Figure 3.7). No differences were observed in the amount of collagen between day 7 and day 14 for the films where no immobilized hTGF- $\beta 1$ was present. In contrast, when the cells were seeded on 


\section{Chapter 3}

TGF- $\beta 1$ activation in hamstring cells through growth factor-binding peptides on polymers

functionalized films with immobilized hTGF- $\beta 1$, a $2.5 \pm 0.4$-fold increase was observed after 14 days for the amount of collagen $(p<0.01)$.

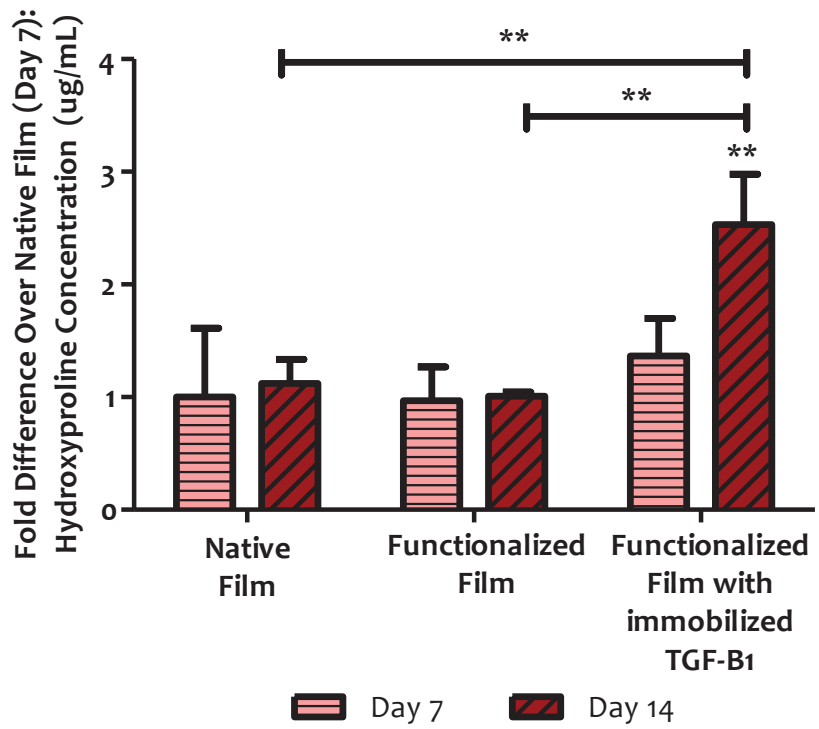

Figure 3.7. Immobilized hTGF-B1 induces the synthesis of collagen in human-derived hamstring cells. ** $p<0.01$ with a two-way ANOVA for the analysis of collagen production over time and a one-way ANOVA with Tukey's post-hoc test for the analysis of collagen production between all conditions at $a$ particular time point. The data are represented as mean \pm SD of triplicates.

\subsubsection{TGF- $\beta 1$-binding peptide captures native circulating TGF- $\beta 1$ leading to} its accumulation on the implanted functionalized polymer

We hypothesized that once implanted in vivo, the TGF- $\beta 1$-binding peptide would capture and accumulate the native circulating TGF- $\beta_{1}$ GFs that are released during the inflammatory stage and induce a more pronounced tissue response around the implant. Both native and functionalized PCL films were subcutaneously implanted in the backs of rats, facing the fascia. Each animal, with a total of three animals per time point, was implanted with both native and functionalized film. At day 3 and day 7 after implantation, samples were harvested and used for immunofluorescence against TGF- $\beta 1$ and histology. Only the samples from day 7 were used for immunofluorescence against TGF- $\beta 1$. Immunostaining against TGF- $\beta 1$ showed a higher fluorescence signal in the films functionalized with the TGF- $\beta 1$-binding peptide when compared with the native films after 7 days of implantation in all three animals (Figure 3.8). This accumulation of the 
native TGF- $\beta 1$ in the films functionalized with the TGF- $\beta 1$-binding peptide led to a higher cellularity around the implant (mostly inflammatory cells) at day 3 when compared with the native films (Figure 3.9A-D). At day 7, much higher amounts of inflammatory and fibroblast-like cells (indicated with yellow arrows) were observed around the functionalized films when compared with the control (Figure 3.9E-H). Additionally, an increased number of blood vessels (indicated with $v$ ) were found around the functionalized PCL implant after 7 days (Figure $3.9 \mathrm{H}$ ). Overall, these results show that the TGF- $\beta 1$ captured by the peptide led to a more pronounced inflammatory response, ultimately leading to the increased recruitment of inflammatory cells, the fibrogenic response and vascularization around the implant.
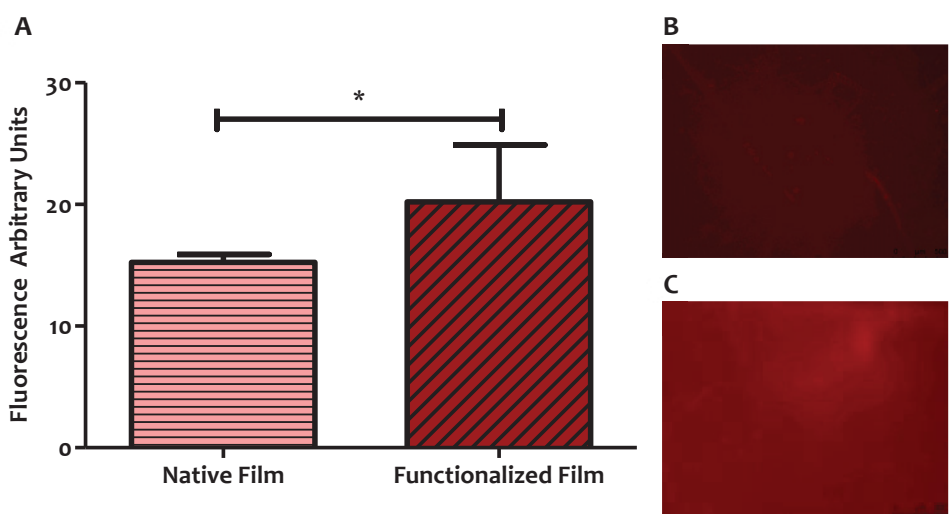

Figure 3.8. (A) Fluorescence quantification of the immunochemistry assay against TGF- 81 immobilized on implanted films. The data represent mean \pm SD of five measurements per sample $(n=3) .{ }^{*} p<0.05$ (two-tailed unpaired t-test). (B) Fluorescence image of the native film implanted subcutaneously in rats. (C) Fluorescence image of the film functionalized with TGF-81-binding peptide implanted subcutaneously in rats. 

Chapter $3 \quad \begin{aligned} & \text { TGF- } \beta 1 \text { activation in hamstring cells through growth factor-binding } \\ & \text { peptides on polymers }\end{aligned}$
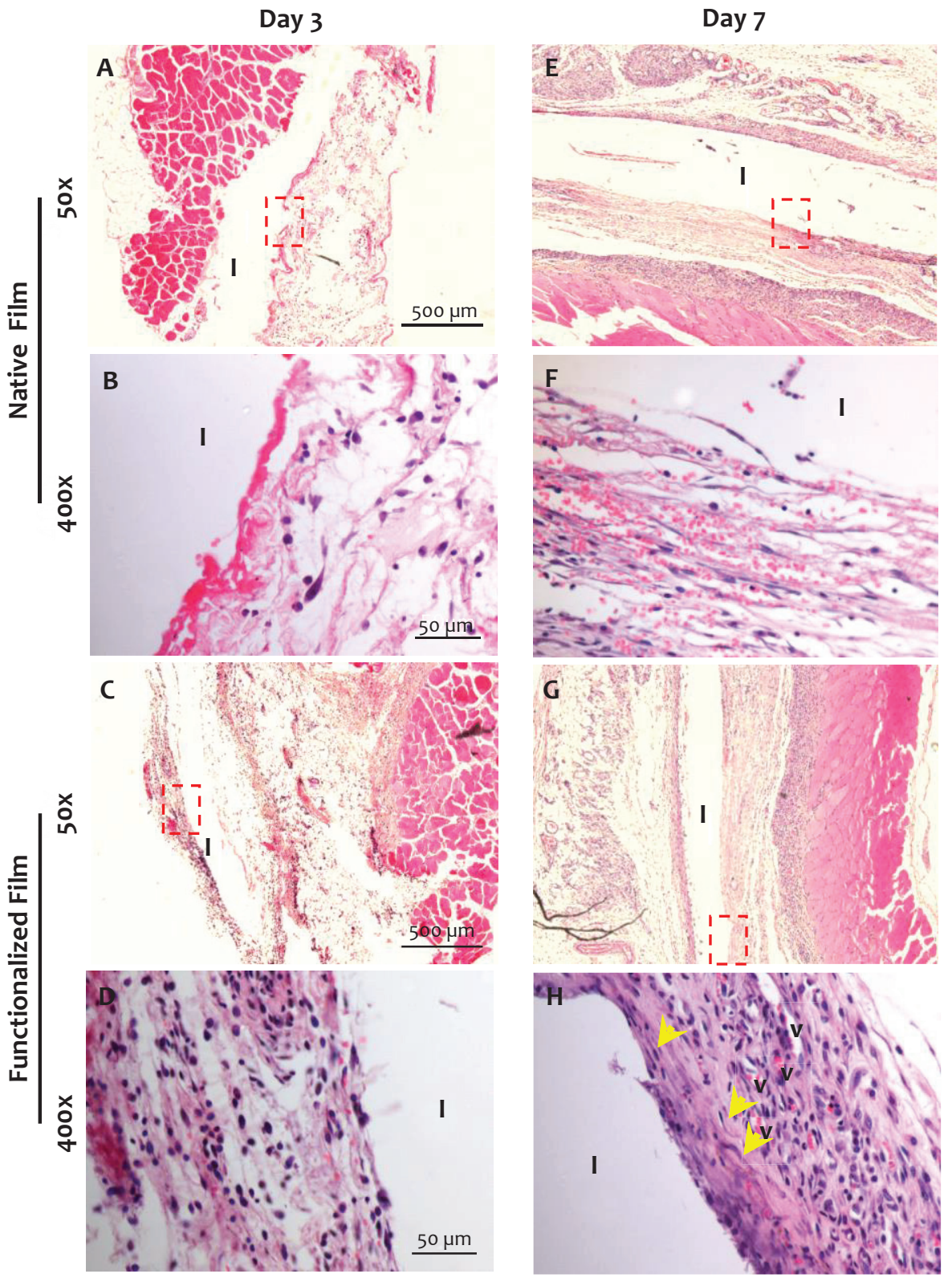

Figure 3.9. Histological evaluation of sample sections after $3(A-D)$ and $7(E-H)$ days of implantation. Haematoxylin and eosin staining on native films (A, B, E and F) and functionalized films (C, G, D and $H$ ). Implant (I), blood vessels ( $v$ ), fibroblast-like cells (yellow arrows), magnified area (red dashed box). 


\subsection{Discussion}

In this work, we hypothesized that the immobilization of hTGF- $\beta 1$ via the interaction with a TGF- $\beta$ 1-binding peptide covalently bound to PCL would allow the presentation of hTGF$\beta 1$ over time and induce a cellular response without needing to add exogenous hTGF- $\beta$ 1. Our study shows that it is possible to non-covalently immobilize hTGF- $\beta 1$ on PCL functionalized with a TGF- $\beta 1$-binding peptide, and that the immobilized hTGF- $\beta 1$ retains its bioactivity by activating target genes and inducing collagen synthesis on humanderived hamstring cells in vitro, and promotes tissue healing in vivo.

TGF- $\beta 1$ is known to play a key role in several cellular mechanisms during wound healing [18]. It is known that TGF- $\beta 1$ is active during all stages of $T / L$ healing, effecting the regulation of cell migration, cell proliferation, stimulation of cell-matrix interactions, collagen synthesis and the termination of cell proliferation [19]. Several studies have indicated that the administration of TGF- $\beta 1$ promotes and accelerates $T / L$ healing. Klein et al. (2002) showed that the administration of TGF- $\beta 1$ significantly increased collagen I and III production in sheath fibroblasts, epitenon and endotenon tenocytes isolated from rabbit flexor tendons [20]. Yahia et al. (1996) demonstrated that TGF- $\beta 1$ increased the production of both collagen and non-collagen proteins in canine ACL fibroblasts [21]. The exogenous administration of TGF- $\beta 1$ in $A C L$ replacement using flexor tendon autograft in dogs increased the bonding strength of the graft to the tunnel wall [5]. Despite the importance of GF during wound healing, soluble administration is not an efficient strategy because it is associated with diffusion problems, low stability of the GF and, to achieve long-term effects, supraphysiological concentrations and systematic administration are often needed. In order to overcome these problems, GF incorporation onto biomaterials can be an alternative to locally delivering the desired GFs in a sustained approach. However, no study has yet been performed assessing the effects of immobilized TGF- $\beta 1$ in T/L repair. Herein, we described a system to non-covalently deliver hTGF- $\beta 1$ and thus accelerate T/L healing. We chose PCL, a biodegradable and FDAapproved polymer, and functionalized it with a previously reported TGF- $\beta 1$-binding peptide in order to immobilize hTGF- $\beta 1$ in the polymer.

We first synthetized the TGF- $\beta$ 1-binding peptide with sequence KGLPLGNSH and acetylated the N-termini. The fact that the covalent immobilization of the TGF- $\beta 1$-binding peptide only occurs through the amine group from the side chain of the lysine amino acid allowed us to achieve a homogeneous orientation of the peptide on the film and consequently of the GFs. This overcame problems related to the heterogeneous 


\section{Chapter 3}

TGF- $\beta 1$ activation in hamstring cells through growth factor-binding peptides on polymers

presentation of the GFs to the cells, which is a major disadvantage of the methods commonly employed for the immobilization of GF. The wettability of the films was used to assess the chemical modifications during the immobilization procedure. The water contact angle for native $\mathrm{PCL}$ films was $77 \pm 2^{\circ}$, consistent with the hydrophobic character of the polymer due to the presence of hydrophobic aliphatic hexane units [22]. After 5 min of oxygen plasma treatment, the hydrophobicity of the film decreased to $39 \pm 5^{\circ}$ due to the appearance of carboxylic and hydroxyl groups on the film, which is in agreement with the literature [23]. Treatment with NHS/EDC led to an increase to $58 \pm 3^{\circ}$ in the contact angle of the film due to the replacement of the hydrophilic carboxylic group by a more hydrophobic ester group, consistent with work previously reported [24]. After incubation of the films containing the amine reactive esters with PBS, no change in the wettability of the film was reported. This was expected since no chemical modification of the film occurred. In contrast, when the films were incubated with the TGF- $\beta 1$-binding peptide in PBS for 4 hours, an increase in the hydrophobicity of the film was reported. This increase in hydrophobicity could be explained by the presence of hydrophobic amino acids such as proline and leucine in the peptide sequence.

The TGF- $\beta 1$-binding affinity of the peptide was further confirmed by immunochemistry and ELISA quantification. From the immunochemistry and ELISA, we concluded that negligible hTGF- $\beta 1$ remained nonspecifically immobilized on the native PCL. In contrast, incubation of the PCL functionalized with a TGF- $\beta 1$-binding peptide with hTGF- $\beta 1$ led to an increase in the fluorescence intensity, which was in agreement with the ELISA quantification data. These results show that the immobilization of the hTGF- $\beta 1$ in the films is solely mediated by interaction with the TGF- $\beta 1$-binding peptide.

The majority of the strategies used to immobilize GFs are associated with a loss of GF bioactivity. In order to confirm that the immobilized hTGF- $\beta 1$ retained its bioactivity, the response of a TGF- $\beta$ reporter cell line to the immobilized hTGF- $\beta 1$ was studied and the intracellular localization of the SMAD2/3 complex in human-derived hamstring cells was assessed. Abe et al. (1994) described for the first time a cell line efficiently transfected with an expression construct containing a PAI-1 promoter fused to the firefly luciferase reporter gene. PAI-1 is involved in the regulation of ECM homeostasis and cell mobility, and is a target gene of TGF- $\beta 1$ [25]. We hypothesized that if the immobilized TGF- $\beta 1$ retained its bioactivity, it would activate the PAI-1 promoter and induce the production of luciferase, which could then be easily quantified using a luciferase assay. No production of luciferase was observed when films without a TGF- $\beta 1$-binding peptide were incubated with hTGF- $\beta 1$ before cell seeding. This can be explained by the fact that these 
films lack affinity for TGF- $\beta 1$, and therefore no hTGF- $\beta 1$ is available to activate the PAI-1 promotor. In contrast, when the films functionalized with a TGF- $\beta 1$-binding peptide and a scramble peptide were incubated with hTGF- $\beta 1$ before the cell seeding, an increase in luciferase activity was observed, reaching a maximum for the film functionalized with a TCF- $\beta$ 1-binding peptide. The scramble peptide may display some residual affinity towards hTGF- $\beta 1$ and consequently immobilize it on the film, leading to the activation of the PAI1 promotor. However, luciferase activity was maximal in the film functionalized with the TGF- $\beta 1$-binding peptide, which can be explained by a higher affinity for hTGF- $\beta 1$ when compared with the scramble peptide and consequently a higher surface concentration of TGF- $\beta 1$ available to the cells. When the cells were seeded in the presence of SB-431452, a specific inhibitor of TGF- $\beta$ type I receptors [26], the luciferase produced in response to the immobilized hTGF- $\beta 1$ was completely suppressed. Upon binding to cell surface receptors type I and II, phosphorylation of the type I receptor by the type II receptor kinases occurs [27]. The activated type I receptor phosphorylates Smad2 and Smad3, which then form a complex with Smad4 [27]. The entire complex translocates into the nucleus where they regulate gene expression [27]. Since the hamstring tendon is one of the most common grafts used for $A C L$ reconstruction, we assessed whether the immobilized TGF- $\beta 1$ was activating the TGF- $\beta$ pathway by following the intracellular localization of the SMAD2/3 complex in human-derived hamstring cells. In fact, when these cells were cultured on functionalized films with immobilized hTGF- $\beta 1$, the SMAD2/3 complex was found in the nucleus, clearly showing activation of the pathway. These results are in agreement with the luciferase assays, showing that the immobilized GFs are bioactive and capable of activating the TGF- $\beta 1$ pathway through the Smad signalling mechanism.

Upon confirmation of the bioactivity of the immobilized TGF- $\beta 1$, we assessed the expression of known TGF- $\beta 1$ target genes in human-derived hamstring cells. Our qPCR results showed an upregulation of both aggrecan and Sox9 in the presence of immobilized hTGF- $\beta 1$ at day 3 , with a downregulation at later time points reaching the values of the films without immobilized hTGF- $\beta 1$. Diez et al. (2009) analysed the effects of TGF- $\beta$ supplementation in developing limb mesenchymal micromass cultures to study the role of TGF- $\beta$ signalling in chondrogenesis and fibrogenesis [28]. It was shown that the addition of TGF- $\beta$ to the cultures downregulated the expression of aggrecan and Sox 9 while upregulating the expression of scleraxis and tenomodulin. It was also shown that blocking Smad with SB-431542 restores the chondrogenic potential of the cultures treated with TGF- $\beta$ s. Our results show that the immobilized hTGF- $\beta 1$ does not induce chondrogenic differentiation in hamstring cells, which could lead to the formation of 
undesired ectopic cartilage in T/L reconstruction. For collagen types I and III, an upregulation at day 3 was also observed in the presence of immobilized hTGF- $\beta 1$. However, for collagen type I, a downregulation was observed at later time points, while in the case of collagen type III, the expression was sustained over time. Our results are in agreement with observations by Klein et al. (2002), where the addition of any TGF- $\beta$ isoform to rabbit tendon cells led to an upregulation of the expression of types I and III collagen [20]. Although collagen type $I$ is the major constituent of $T / L$, the downregulation observed at later time points for collagen type I in the presence of immobilized hTGF- $\beta 1$ may be beneficial for the wound healing, since mature scar tissue is formed from collagen type I [29]. While not being a major component of $\mathrm{T} / \mathrm{L}$, collagen type III is crucial for tissue healing due to its ability to rapidly crosslink and stabilize the repair site [30]. One study showed that subcutaneous wounds in collagen type IIIdeficient mice had significantly more scar tissue area at 21 days post wound compared to wild-type mice [31]. In conclusion, the downregulation of collagen type I observed over time in the presence of immobilized TGF- $\beta 1$ may reduce the formation of scar tissue, which would compromise the biomechanical properties of the tissue, whereas the sustained expression of collagen type III could help in stabilizing the wound site and promote graft-to-bone integration. From the hydroxyproline quantification, we confirmed that the upregulation of collagen gene levels in response to the immobilized hTGF- $\beta 1$ was being translated to a higher content of collagen protein.

Despite the known role of GFs in the healing process, only BMP-2 and BMP-7 are currently FDA approved to be used in regeneration therapies. The unknown effect of the administration of GFs, a lack of suitable delivery vehicles that lower the amount of GFs needed and the costs associated with these therapies are all drawbacks that must be optimized in order to implement an efficient and safe therapy based on these proteins. In this study, we hypothesized that functionalizing biomaterials with a TGF- $\beta 1$-binding peptide would turn the implanted biomaterial into an anchor point for the endogenous TGF- $\beta 1$, leading to its accumulation, consequently enhancing the healing response of the surrounding tissue without needing to administer exogenous TGF- $\beta$. Such a strategy would eventually reduce the risks associated with the administration of high doses of GFs and consequently increase the safety and decrease the associated costs. TGF- $\beta 1$ is known to have a role in all stages of wound healing. Upon cutaneous injury, a blood clot forms to stop the blood leaking from the damaged blood vessels. Platelets within this clot release several cytokines, including TGF- $\beta 1$ [32]. In turn, this TGF- $\beta 1$ will attract inflammatory cells, such as leukocytes and macrophages, which will release more TGF- $\beta 1$ and initiate the inflammatory phase. After inflammation, the tissue formation phase 
commences, which includes events such as fibroblast activation, ECM remodelling and neovascularization [33]. The final phase of the healing process is the tissue remodelling phase, during which changes in both the epidermis and dermis will occur, ultimately leading to the formation of a scar at the injury site. To test this hypothesis, films of native and functionalized PCL were subcutaneously implanted in the backs of rats. After 3 and 7 days, the implants were harvested and histologically stained. The implants harvested 7 days after implantation were also immunostained against TGF- $\beta 1$. From our immunochemistry against TGF- $\beta 1$, a higher fluorescence signal was reported in the functionalized PCL, showing that the TGF- $\beta 1$-binding peptide captured the TGF- $\beta 1$ released during the inflammation, which led to its accumulation on the functionalized film. This accumulation eventually led to a more pronounced healing response at the injury site. There was a higher cellularity around the functionalized films in all the animals at day 3, which was largely composed of inflammatory cells. After 7 days, the cellularity was once again higher around the functionalized films. In addition to inflammatory cells, fibroblast-like cells and a denser blood vessel network were, after 7 days, solely found around the functionalized PCL. In conclusion, these results show that the TGF- $\beta 1$-binding peptide captured the endogenous TGF- $\beta 1$, leading to its accumulation on the PCL. This higher local accumulation of TGF- $\beta 1$ induced a more pronounced inflammatory response due to the recruitment of more inflammatory cells to the injury site, which consequently led to a more robust tissue formation phase, characterized by the recruitment of fibroblast cells and neovascularization. These results show the potential of using the TGF- $\beta 1$-binding peptide in the design and improvement of biomedical devices, giving them the capacity to function as an anchor point for the patient's natural circulating TGF$\beta 1$. This would allow the TGF- $\beta 1$ that is naturally circulating or that is produced and released during the inflammatory stage to be captured and consequently to locally enhance its concentration, leading to a more robust healing process without needing to add exogenous GFs. 


\subsection{Conclusions}

A strategy to immobilize hTGF- $\beta 1$ in PCL has been detailed through the interaction with a TGF- $\beta 1$-binding peptide. The immobilized hTGF- $\beta 1$ presented to the cells is bioactive and capable of activating the TGF- $\beta$ pathway in primary human-derived hamstring cells. This is the first time that a method for the immobilization and delivery of hTGF- $\beta 1$ for T/L healing has been described. Our in vitro studies showed that the immobilized hTGF- $\beta 1$ induced the expression of collagen types I and III in human-derived hamstring cells without effecting chondrogenic related genes (Sox9 and aggrecan). In vivo studies showed that there was a higher accumulation of native TGF- $\beta 1$ on the films functionalized with a TGF- $\beta 1$-binding peptide, which led to a more pronounced recruitment of inflammatory cells, a fibrotic response and neovascularization around the implant. This highlights the potential of using this peptide sequence in the design of improved medical devices through adding the capacity to capture the patient's native TGF- $\beta 1$ to the device and therefore locally enhance the effect that this GF has in tissue repair.

\subsection{Acknowledgments}

We would like to acknowledge Smith\&Nephew for providing financial support, and Dr. Bruma Fu and Angel Lee from the University of Hong Kong for their collaboration in the in vivo experiments. We would also like to acknowledge Joyce Mulder for her contribution to the laboratory work. 


\subsection{References}

1. Surgeons, A.A.o.O. Anterior Cruciate Ligament Injury: Surgical Considerations. 2009 [cited 2005 July]; Available from: http://orthoinfo.aaos.org/topic.cfm?topic=a00297.

2. Singhal MC, Gardiner JR, and Johnson DL, Failure of primary anterior cruciate ligament surgery using anterior tibialis allograft. Arthroscopy, 2007. 23(5): p. 469-75.

3. Lee K, Silva EA, and Mooney DJ, Growth factor delivery-based tissue engineering: general approaches and a review of recent developments. J R Soc Interface, 2011. 8(55): p. 153-70.

4. Masters KS, Covalent growth factor immobilization strategies for tissue repair and regeneration. Macromol Biosci, 2011. 11(9): p. 1149-63.

5. Yamazaki S, et al., The Effect of Transforming Growth Factor-81 on Intraosseous Healing of Flexor Tendon Autograft Replacement of Anterior Cruciate Ligament in Dogs. Arthroscopy, 2005. 21(9): p. 1034-1041.

6. Katsura T, et al., Effects of administration of transforming growth factor (TGF)-beta1 and anti-TGF-beta1 antibody on the mechanical properties of the stress-shielded patellar tendon. J Biomech, 2006. 39(14): p. 2566-72.

7. Cabanas-Danés J, Huskens J, and Jonkheijm P, Chemical strategies for the presentation and delivery of growth factors. J. Mater. Chem. B, 2014. 2(17): p. 2381-2394.

8. Sahoo S, Toh SL, and Goh JC, A bFGF-releasing silk/PLGA-based biohybrid scaffold for ligament/tendon tissue engineering using mesenchymal progenitor cells. Biomaterials, 2010. 31(11): p. 2990-8.

9. Kimura $\mathrm{Y}$, et al., Regeneration of anterior cruciate ligament by biodegradable scaffold combined with local controlled release of basic fibroblast growth factor and collagen wrapping. Tissue Eng Part C Methods, 2008. 14(1): p. 47-57.

10. Sahah RN, et al., Supramolecular design of self-assembling nanofibers for cartilage regeneration. PNAS, 2009. 107(8): p. 3293-3298.

11. Abe $M$, et al., An assay for transforming growth factor-beta using cells transfected with a plasminogen activator inhibitor-1 promoter-luciferase construct. Anal Biochem, 1994. 216(2): p. 276-84.

12. Ghebes CA, et al., Anterior cruciate ligament- and hamstring tendon-derived cells: in vitro differential properties of cells involved in ACL reconstruction. J Tissue Eng Regen Med, 2015.

13. Nakao A, et al., TGF-beta receptor-mediated signalling through Smad2, Smad3 and Smad4. EMBO J, 1997. 16(17): p. 5353-5362.

14. Wilson CG, Nishimuta JF, and Levenston ME, Chondrocytes and meniscal fibrochondrocytes differentially process aggrecan during de novo extracellular matrix assembly. Tissue Eng Part A, 2009. 15(7): p. 1513-22.

15. Furumatsu T, Ozaki T, and Asahara H, Smad3 activates the Sox9-dependent transcription on chromatin. Int J Biochem Cell Biol, 2009. 41(5): p. 1198-204.

16. Reed MJ, et al., TGF-beta 1 induces the expression of type I collagen and SPARC, and enhances contraction of collagen gels, by fibroblasts from young and aged donors. J Cell Physiol, 1994. 158(1): p. 169-79.

17. Kenyon NJ, et al., TGF-beta1 causes airway fibrosis and increased collagen I and III mRNA in mice. Thorax, 2003. 58(9): p. 772-7.

18. Penn JW, Grobbelaar AO, and Rolfe KJ, The role of the TGF- 8 family in wound healing, burns and scarring: a review. Int J Burns Trauma, 2012. 2(1): p. 18-28. 
19. Molloy T, Wang Y, and Murrell G, The roles of growth factors in tendon and ligament healing. Sports Med, 2003. 33(5): p. 381-94.

20. Klein MB, et al., Flexor tendon healing in vitro: effects of TGF-beta on tendon cell collagen production. J Hand Surg Am, 2002. 27(4): p. 615-20.

21. DesRosiers EA, Yahia L, and Rivard $\mathrm{CH}$, Proliferative and matrix synthesis response of canine anterior cruciate ligament fibroblasts submitted to combined growth factors. J Orthop Res, 1996. 14(2): p. 200-8.

22. Wurth JJ, Blumenthal NR, and Shastri VP, Hydrophilization of Poly(Caprolactone) Copolymers through Introduction of Oligo(Ethylene Glycol) Moieties. PLoS One, 2014. 9(6).

23. Jokinen V, Suvanto P, and Franssila S., Oxygen and nitrogen plasma hydrophilization and hydrophobic recovery of polymers. Biomicrofluidics, 2012. 6(1): p. 16501-165011.

24. Zander NE, et al., Surface-modified nanofibrous biomaterial bridge for the enhancement and control of neurite outgrowth. Biointerphases, 2010. 5(4): p. 149-158.

25. Kutz SM, et al., TGF-beta1-induced PAl-1 gene expression requires MEK activity and cell-tosubstrate adhesion. J Cell Sci, 2001. 114(21): p. 3905-14.

26. Halder SK, Beauchamp RD, and D. PK, A Specific Inhibitor of TGF-B Receptor Kinase, SB431542, as a Potent Antitumor Agent for Human Cancers. Neoplasia, 2005. 7(5): p. 509-521.

27. Derynck R and Zhang YE, Smad-dependent and Smad-independent pathways in TGF-beta family signalling. Nature, 2003. 425(6958): p. 577-84.

28. Lorda-Diez Cl, et al., Transforming growth factors beta coordinate cartilage and tendon differentiation in the developing limb mesenchyme. J Biol Chem, 2009. 284(43): p. 2998896.

29. Hardy MA, The biology of scar formation. Phys Ther, 1989. 69(12): p. 1014-24.

30. Liu SH, et al., Collagen in tendon, ligament, and bone healing. A current review. Clin Orthop Relat Res, 1995(318): p. 265-78.

31. Volk SW, et al., Diminished type III collagen promotes myofibroblast differentiation and increases scar deposition in cutaneous wound healing. Cells Tissues Organs, 2011. 194(1): p. 25-37.

32. Wang XJ, et al., Role of TGF beta-mediated inflammation in cutaneous wound healing. J Investig Dermatol Symp Proc, 2006. 11(1): p. 112-7.

33. Velnar T, Bailey T, and S. V, The wound healing process: an overview of the cellular and molecular mechanisms. J Int Med Res, 2009. 37(5): p. 1528-42. 


\subsection{Supporting information}

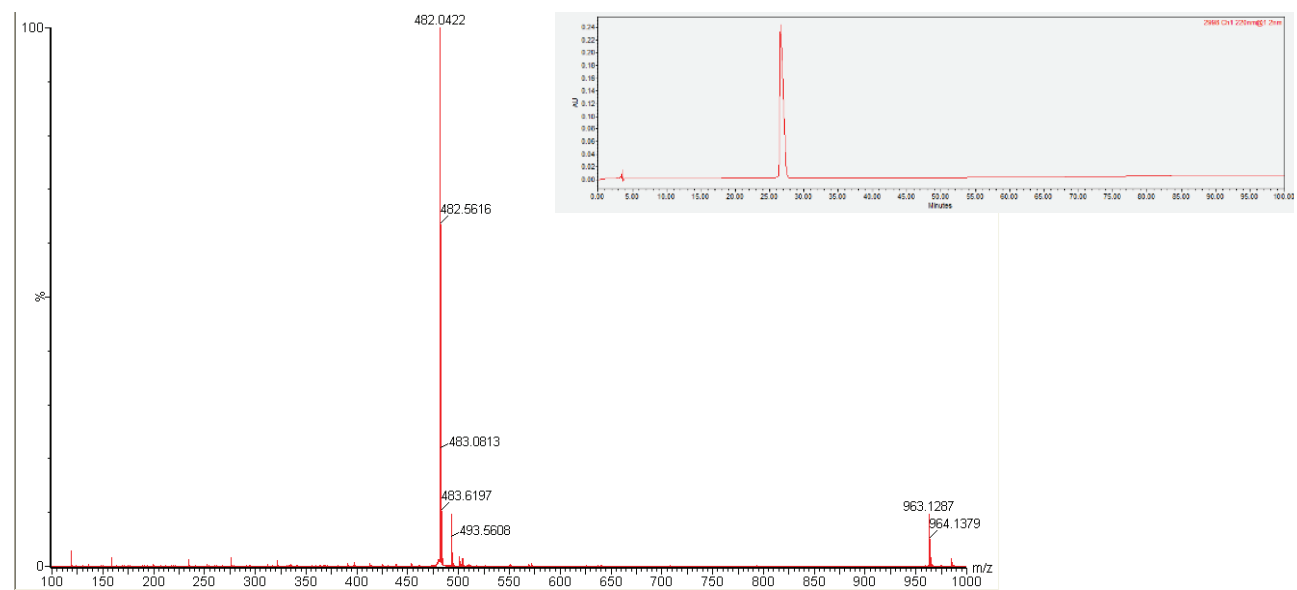

Figure S3.1. Mass spectroscopy of the TGF-61-binding peptide after purification and respective analytical HPLC.

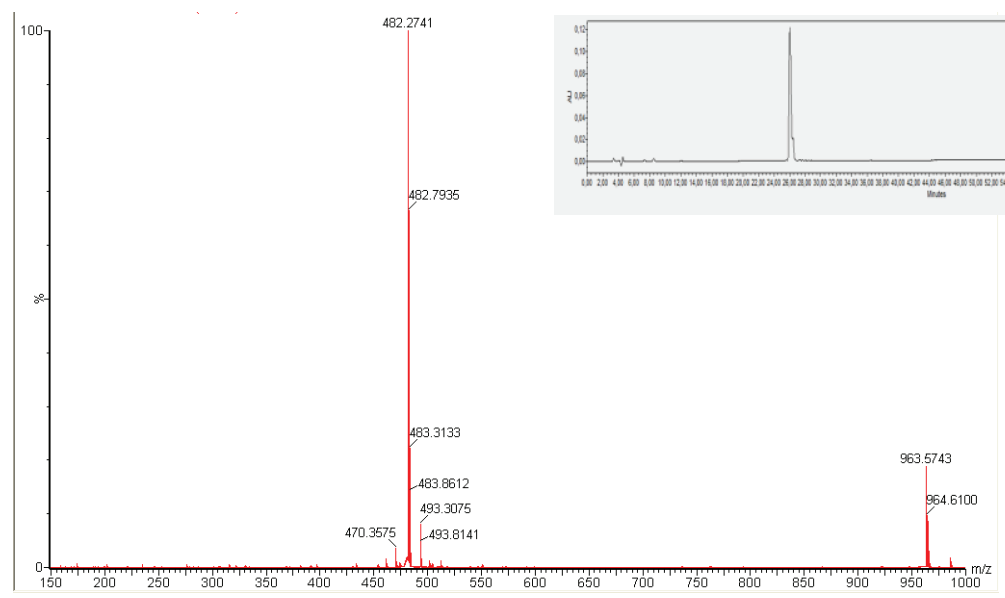

Figure S3.2. Mass spectroscopy of the TGF-B1 scramble peptide after purification and respective analytical HPLC. 


\section{Chapter 4}

\section{Immobilization of hBMP-2 on polymers directs cell fate}

From all of the known growth factors (GFs) involved in tissue healing, only human bone morphogenetic protein-2 and -7 (hBMP-2 and -7) are currently approved for clinical use. The clinical products involving hBMP-2 and -7 delivery consist of collagen sponges that adsorb the GFs. Since the adsorption of the BMP to the collagen sponge is based on weak interactions, huge amounts of GFs must be used in order to induce bone repair, which raises several risks associated with the use of supraphysiological amounts of BMP. The use of stronger interactions between the biomaterial and GFs can prolong the exposure of the GF to the environment, consequently lowering the amount of GF required and avoiding the risks associated with the administration of high doses of GF. In the work described here, the use of a synthetic peptide with an affinity for BMP-2 was studied as a vehicle to immobilize and deliver the GF. Polycaprolactone (PCL) films were functionalized with the BMP-2binding peptide and its affinity for hBMP-2 was confirmed by immunochemistry. Moreover, cell experiments showed that the immobilized hBMP-2 retained its bioactivity and supported osteogenic differentiation of $\mathrm{C}_{2} \mathrm{C}_{12}$ cells, leading to a 10.7-fold increase in the synthesis of ALP. When the peptide-functionalized films were subcutaneously implanted in rats, a higher accumulation of BMP-2 was observed than with the native films. This accumulation was translated into a higher cellularity and vascularization around the implant at day 3 and a higher matrix deposition at day 7 after implantation. These results show the potential for the use of this synthetic peptide in the delivery or capture of endogenous BMP-2, which would overcome the problems associated with the current commonly-used delivery strategies. 


\subsection{Introduction}

GFs are involved in the regulation of several cellular processes such as migration, differentiation, proliferation and healing [1]. One of the most important classes of GF is the transforming growth factor $\beta$ (TGF- $\beta$ ) superfamily, which includes TGF- $\beta 1-3$, activins and BMPs [2]. To date, more than 20 different BMPs have been identified in humans, having different roles in processes such as embryogenesis, skeletal formation and healing and cell differentiation [3]. BMPs are synthetized and released by different cell types such as osteoprogenitor cells, osteoblasts, chondrocytes and platelets [4-7]. Upon release, their biological function depends on several parameters of the microenvironment, such as target cell type, extracellular matrix composition, concentration and the presence of other biological molecules that can modulate the cell's response to BMPs $[8,9]$. The importance of BMPs in bone healing relies on the fact that they induce the differentiation of mesenchymal progenitor cells into osteoblasts, which deposit bone matrix [10]. However, of the 20 different BMPs identified in humans, only BMP-2 and BMP-7 are currently applied in bone healing procedures [11]. The clinical products that make use of these two BMPs consist of a collagen sponge in which the BMP is adsorbed and subsequently released by diffusion in time [12]. Immobilization of GFs overcomes problems associated with diffusion and stability in the body, with physical adsorption of GFs to biomaterials being one of the many possible immobilization strategies [13]. However, since this method relies on weak interactions, it is often associated with an initial burst release and, consequently, demands supraphysiological doses of GF in order to elicit a biological response. The use of high doses of BMP is associated with several adverse effects, such as ectopic bone formation, cyst-like bone void formation and swelling of the tissue, ultimately compromising the quality of the newly-formed bone [14]. Improving the release profile of BMPs and the retention of BMPs at the injury site could potentially enhance the safety and efficacy and, concomitantly, reduce costs as a consequence of decreasing the amount of GF required. Immobilization strategies that employ stronger interactions between GFs and biomaterials can prolong the presentation and availability of the GF in the injury site and consequently reduce the initial amount of GF required to induce a tissue response [15]. One study showed that covalent immobilization of BMP-2 to a self-assembled monolayer (SAM) led to the phosphorylation of Smad1/5/8, a hallmark of the activation of BMP signalling, and induced the osteogenic differentiation of $\mathrm{C}_{2} \mathrm{C}_{12}$ cells [16]. Overexposure to the covalently-immobilized GF and loss of bioactivity during the immobilization procedure are some disadvantages associated with this strategy [17]. Non-covalent immobilization of GFs can also be achieved through interaction with extracellular matrix 
components such as heparin [18]. One study compared the use of a collagen sponge, the formulation used by the commercially available product, and a heparin-conjugated fibrin as delivery vehicles of BMP-2 to mouse calvarial defects [19]. They reported that in the heparin-conjugated fibrin, less than half of the amount of BMP-2 used in the collagen sponge was necessary to achieve the same level of bone regeneration. They claimed that sustained release of BMP-2 during a sufficiently long period in the heparin-conjugated fibrin resulted in enhanced bone healing. This was achieved without needing to use supraphysiological amounts of BMP-2, which therefore reduced the risks associated with the high dosage of BMP-2. In this work, hBMP-2 was specifically immobilized via the interaction with a BMP-2-binding peptide attached to PCL films (Figure 4.1). The amino acid sequence of the peptide involved in the affinity towards BMP-2 had been previously identified using a phage display screening, with a reported binding constant of $1.9 \pm 0.9$ $x 10^{6} \mathrm{M}^{-1}[20]$. Here we confirmed the interaction of the BMP-2-binding peptide towards hBMP-2 by immunochemistry (Figure 4.2). We observed an upregulation in the amount of luciferase produced in a BMP reporter cell line in response to the immobilized hBMP2, clearly showing that the immobilized hBMP-2 retained its bioactivity (Figure 4.4). Immobilized hBMP-2 also led to a 10.7-fold increase in ALP expression in C2C12 cultured in the PCL films (Figure 4.6). Films functionalized with the BMP-2-binding peptide captured the endogenous GF when subcutaneously implanted in rats (Figure 4.7). Overall, this led to a higher cellularity and to the appearance of blood vessel-like structures at day 3, and increased matrix deposition at day 7 after implantation (Figure 4.8). To our knowledge, this is the first time that a synthetic peptide has been used to immobilize and deliver hBMP-2 to cells. 


\subsection{Materials and methods}

\subsubsection{Materials}

$\mathrm{N}, \mathrm{N}, \mathrm{N}^{\prime}, \mathrm{N}^{\prime}$-Tetramethyl-O-(1H-benzotriazol-1-yl)uronium hexafluorophosphate (HBTU) was obtained from MultiSynTech. Chloroform and 1-methyl-2-pyrrolidinone (NMP) were purchased from WR Chemicals. $\mathrm{NaOH}$ was obtained from Riedel-de Haën. All other reagents or products were purchased from Sigma-Aldrich unless noted otherwise.

\subsubsection{Peptide synthesis and purification}

The peptide sequences were synthetized using Fmoc-solid phase peptide synthesis on a Syro II MultiSynTech automated peptide synthesizer. The BMP-2-binding and scrambled peptides with sequences KGYPVHPST and KGTPVHYPS, respectively, were prepared on Fmoc-Rink 4-methylbenzhydrylamine (MBHA) resin (MultiSynTech GmBH, 50 mg scale, substitution $0.52 \mathrm{mmol} / \mathrm{g}$ ), using $0.26 \mathrm{M}$ of HBTU, $0.52 \mathrm{M}$ of $\mathrm{N}, \mathrm{N}$-diisopropylethylamine (DIPEA), $2 \mathrm{M}$ of piperidine and $0.29 \mathrm{M}$ of each amino acid. The $\mathrm{N}$-termini of the final peptide sequences were manually acetylated in 16\% (v/v) acetic anhydride, 30\% (v/v) DIPEA and $54 \%(v / v)$ NMP for one hour at room temperature. The peptides were cleaved from the resin and amino acid side groups were deprotected using 95\% (v/v) trifluoroacetic acid, $2.5 \%(\mathrm{v} / \mathrm{v})$ triisopropylsilane and $2.5 \%(\mathrm{v} / \mathrm{v})$ milliQ water. The peptides were then collected by precipitation in cold diethyl ether and the organic solvents were removed in a rotatory evaporator. The peptides were redissolved in milliQ water and lyophilized overnight. The resulting products were purified using standard preparative HPLC methods. MS (ESI): $\mathrm{m} / \mathrm{z}=1026.3[\mathrm{M}+\mathrm{H}]+\left(\right.$ calculated 1026.1 for $\left.\mathrm{C}_{47} \mathrm{H}_{71} \mathrm{~N}_{13} \mathrm{O}_{13}\right)$ for KGYPVHPST. MS (ESI): $\mathrm{m} / \mathrm{z}=1026.8[\mathrm{M}+\mathrm{H}]+$ (calculated 1026.1 for $\mathrm{C}_{47} \mathrm{H}_{71} \mathrm{~N}_{13} \mathrm{O}_{13}$ ) for KGTPVHYPS.

\subsubsection{Preparation of PCL films displaying BMP-2-binding peptides}

A $12.5 \%(w / v)$ solution of PCL in chloroform was prepared and homogenized by sonication. The homogenized solution was cast in a petri dish, pre-silanized with a PFDTS $(1 \mathrm{H}, 1 \mathrm{H}, 2 \mathrm{H}, 2 \mathrm{H}$-perfluorodecyltrichlorosilane, $\geq 97 \%, \mathrm{ABCR} \mathrm{GmbH})$ anti-sticky layer, and the solvent allowed to evaporate overnight. The polymer was melted, allowed to again solidify and was then cut into circular films with a diameter of $21 \mathrm{~mm}$ in order to fit inside the wells of a 12-well plate. The individual circular films were extensively washed with demi-water and milliQ water and dried with a $\mathrm{N}_{2}$ stream. The dried films were exposed to oxygen plasma for $5 \mathrm{~min}$ (at an oxygen pressure of $1.0 \mathrm{bar}$, a vacuum pressure of 200 
mbar and a current of $40 \mathrm{~A}$ ) and subsequently immersed in a $1 \mathrm{M} \mathrm{NaOH}$ solution for one hour with gentle agitation. PCL films were then washed and dried as mentioned above, and incubated with a solution of $50 \mathrm{mM}$ 1:1 NHS/EDC in MES buffer for one hour with agitation. PCL films were washed and dried again as mentioned above and incubated with $1 \mathrm{mM}$ of the peptide in phosphate buffered saline (PBS) for 4 hours with agitation. Films were then extensively washed with PBS and sterilized by incubating the films overnight in a solution of $10 \%$ penicillin/streptomycin (Life Technologies) in PBS prior to cell seeding

\subsubsection{Water contact angle measurements}

The wettability of the PCL films was determined by a drop contact angle system (Krüss Contact Angle Measuring System G10). The contact angle was measured and calculated using Drop Analysis software. All reported contact angles are the average of $n=6$ measurements. MilliQ water was used to measure the contact angle of the films.

\subsubsection{BMP-2-binding and immunofluorescence}

The PCL films were incubated with $1 \mu \mathrm{g} / \mathrm{mL}$ of hBMP-2 (PeproTech) in PBS for one hour with gentle agitation. The films were then washed three times for 10 min with $1 \mathrm{mM}$ PBS tween-20 (PBST) and then with PBS for a further $10 \mathrm{~min}$. Next, the films were blocked for one hour with PBS containing $1 \%(\mathrm{w} / \mathrm{v})$ bovine serum albumin (BSA) and subsequently washed as described above. Afterwards, the films were incubated with a $2 \mu \mathrm{g} / \mathrm{mL}$ solution of the primary antibody (rabbit polyclonal anti-human BMP-2, PeproTech) in the blocking solution for one hour with agitation. The films were washed as mentioned above and then incubated with an $8 \mu \mathrm{g} / \mathrm{mL}$ solution of the secondary antibody (goat antirabbit Alexa Fluor 594, Invitrogen) in PBS containing 1\% w/v BSA for one hour with gentle agitation. Prior to fluorescence microscopy, the films were washed three times for 10 min with $1 \mathrm{mM}$ PBST, rinsed three times with PBS and dried under a N2 stream.

For cell experiments, the sterile films were washed three times with PBS and incubated with concentrated hBMP-2 in PBS for one hour with gentle agitation. Subsequently, the films were extensively washed with TBST (0.5\%) and PBS to remove any traces of the washing buffer prior to cell seeding. 


\subsubsection{Cell culture}

$\mathrm{C}_{2} \mathrm{C}_{12}$ Luc (a kind gift from Daniel Rifkin's lab) and C2 $\mathrm{C}_{12}$ were expanded in Dulbecco's Modified Eagle Medium (DMEM, Life Technologies, Gaithersburg, MD) supplemented with $20 \%$ Fetal Bovine Serum (FBS, Life Technologies), $100 \mathrm{U} / \mathrm{mL}$ penicillin (Life Technologies), $100 \mu \mathrm{g} / \mathrm{mL}$ streptomycin (Life Technologies) and $2 \mathrm{mM}$ L-glutamine (Life Technologies). Cells were grown at $37^{\circ} \mathrm{C}$ in a humid atmosphere with $5 \% \mathrm{CO}_{2}$. The medium was refreshed twice per week and cells were used for further subculturing or cryopreservation on reaching near confluence. C2C12 Luc express luciferase under the control of a ID1 promoter - a BMP-2 target gene [21].

\subsubsection{Luciferase assay}

C2 12 Luc were seeded on films at 16,000 cells $/ \mathrm{cm}^{2}$ in DMEM medium supplemented with $10 \% \mathrm{FBS}$ and allowed to attach overnight at $37^{\circ} \mathrm{C}$ in a $5 \% \mathrm{CO}_{2}$ incubator. The cells were lysed the following day and the luciferase quantified according to the manufacturer's protocol (Promega, E4530). Luciferase values were normalized for DNA content quantified by CyQUANT cell proliferation assay (Invitrogen).

\subsubsection{ALP quantification and staining}

For the analysis of the amount of collagen produced, hamstring cells were seeded on films at 5,000 cells $/ \mathrm{cm}^{2}$ and cultured for 7 and 14 days in culture medium. Hydroxyproline quantification was used as a direct method for the determination of the collagen content of the samples. Cells were washed with PBS, lysed with $12 \mathrm{~N} \mathrm{HCl}$ and scratched from the films. The lysate was transferred to a pressure-tight Teflon-capped vial and hydrolysed at $120^{\circ} \mathrm{C}$ for three hours. After hydrolyzation, the amount of hydroxyproline was quantified using the Hydroxyproline Colorimetric Assay Kit (BioVision) according to the manufacturer's instructions.

\subsubsection{Subcutaneous implantation rat model}

All of the animal experiments were approved by the animal research ethics committee of the Chinese University of Hong Kong. Eight 12-week-old Sprague Dawley male rats were used in this study. The rats were anesthetized by intraperitoneal injection of $10 \%$ ketamine $/ 2 \%$ xylazine (Kethalar, $0.3 \mathrm{ml}: 0.2 \mathrm{ml}$ ); sedation was maintained by intramuscular injection of $10 \%$ ketamine (Sigma Chemical CO, St. Louis, MO). Subcutaneous implantation of PCL was performed. In brief, once the animals were 
anesthetized, shaved and washed, two incisions were made and native $P C L$ and $P C L$ functionalized with a BMP-2-binding peptide were inserted into the pockets and fixed to the fascia. The skin wound was then closed using sutures. At day 3 and day 7 post implantation, the animals were sacrificed and samples were harvested. Samples from the subcutaneous rat model were harvested at day 7 post implantation and rinsed with PBS. Harvested samples were fixed with 10\% buffered formalin for 10 min and further permeabilized with PBST for 15 min. Samples were then washed with PBS and blocked with $1 \%(w / v)$ BSA for 1 hour in a shaker. Next, the samples were washed three times for 10 min with PBST and incubated with a dilution of 1:100 of the primary antibody (rabbit polyclonal anti BMP-2, Abcam) overnight at $4^{\circ} \mathrm{C}$. After primary antibody incubation, samples were washed with PBST and incubated with a dilution of 1:100 of the secondary antibody (goat anti rabbit IgG-PE, Santa Cruz Biotechnology) for one hour at room temperature. Samples were washed with PBST prior to imaging.

\subsubsection{Histology}

At day 3 and day 7 post implantation, PCL implants and the surrounding tissues were harvested and rinsed with PBS. Harvested samples were fixed with $10 \%$ buffered formalin overnight and embedded in paraffin. Subsequently, $5 \mu \mathrm{m}$-thick paraffin sections along the transverse plane were collected. Haematoxylin and eosin (H\&E) stained sections were examined under light microscopy (Leica Microsystems, Wetzlar, Germany).

\subsubsection{Statistical analysis}

The data were analysed using a Student's paired t-test, a one-way analysis of variance followed by a Tukey's multiple comparison test ( $p<0.05)$, or a two-way analysis of variance. The values represent the mean and standard deviation of three independent measurements. 


\subsection{Results}

\subsubsection{Functionalization of PCL with BMP-2-binding peptides}

The BMP-2-binding sequence, YPVHPST, was synthesized with an extra glycine and lysine at the N-termini (Figure S4.1). The function of the extra amino acids was previously reported in chapter 3. The chemical modifications of the surface of the films were confirmed by following the changes in the water contact angle (Figure 4.1). Treatment of the native $P C L$ films with oxygen plasma led to a decrease in the water contact angle of $39 \pm 1^{\circ}(p<0.0001)$ due to the introduction of oxygen-containing groups to the surface. An increase of $19 \pm 2^{\circ}$ ( $\left.p<0.0001\right)$ was observed after incubating the oxygen plasma activated films with NHS/EDC. Incubation of the amine reactive esters with $1 \mathrm{mM}$ of the BMP-2-binding peptide in PBS resulted in an increase of the water contact angle of $5 \pm 1^{\circ}$ $(p<0.01)$. In contrast, incubation with only PBS did not lead to a significant change in the water contact angle of the films with the amine reactive esters.

A
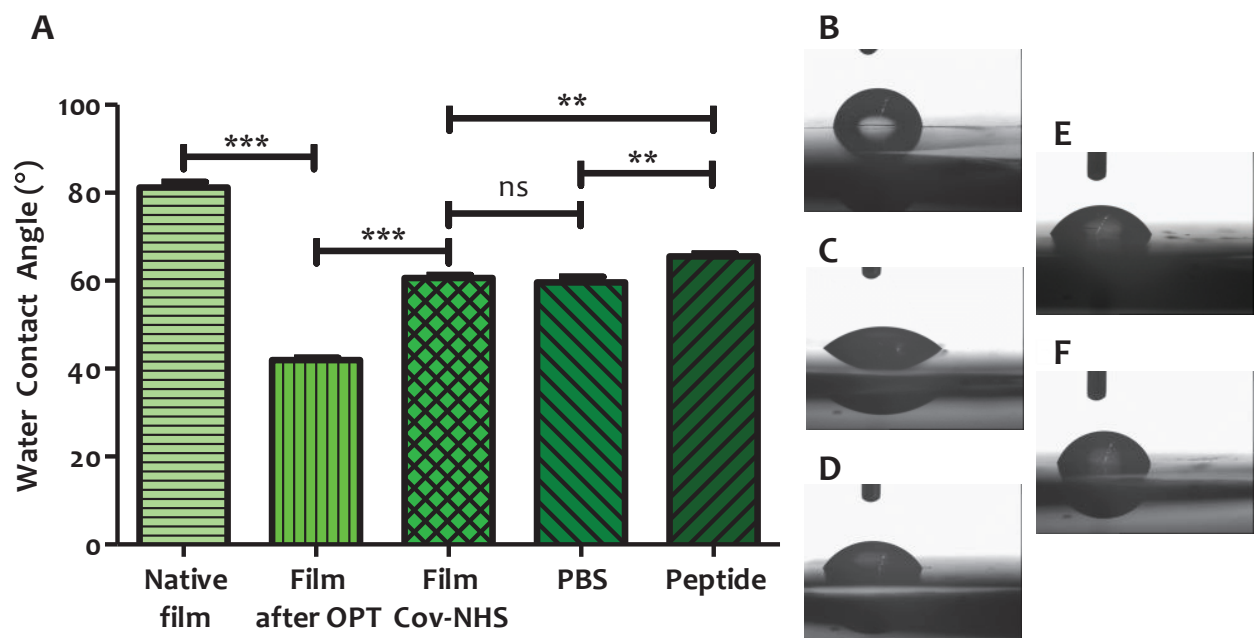

Figure 4.1. (A) Water contact angle measurements with respective representative pictures of the films during the chemical functionalization procedure; (B) 'Native films' are the PCL films without any chemical modification; (C) 'Film after OPT' refers to the native PCL films exposed for 5 min to oxygen plasma; (D) 'Films Cov-NHS' are the films with amine reactive esters after 1 hour incubation with NHS/EDC; (E) 'PBS' indicates the films with reactive amine esters incubated for 4 hours with PBS; (F) 'Peptide' refers to the films with reactive amine esters incubated for 4 hours with a $1 \mathrm{mM}$ peptide in PBS. $* * * p<0.0001$ (two-tailed unpaired t-test). The data represent the mean $\pm S D$ of two measurements per sample $(n=6)$. 


\subsubsection{BMP-2 immobilization on the functionalized PCL films}

An immunostaining against hBMP-2 was performed in order to confirm the binding of the GF to the functionalized films. Our results showed that the fluorescence intensity was higher on functionalized films incubated with hBMP-2 compared to nonfunctionalized controls (Figure 4.2A and B; $\mathrm{p}<0.0001$ ). From our results, we can also conclude that the reported signal is not due to nonspecific interaction of the primary or secondary antibody with the functionalized films. Incubation of the native film with hBMP-2 also led to a significant increase in the fluorescence signal when compared with the native film without hBMP-2 incubation. This can be explained by the fact that part of the hBMP-2 nonspecifically binds onto the native films. In summary, functionalization of the films with a BMP-2-binding peptide led to a higher surface concentration of hBMP-2 due to its affinity for the BMP-2-binding peptide, resulting in the highest fluorescence signal.

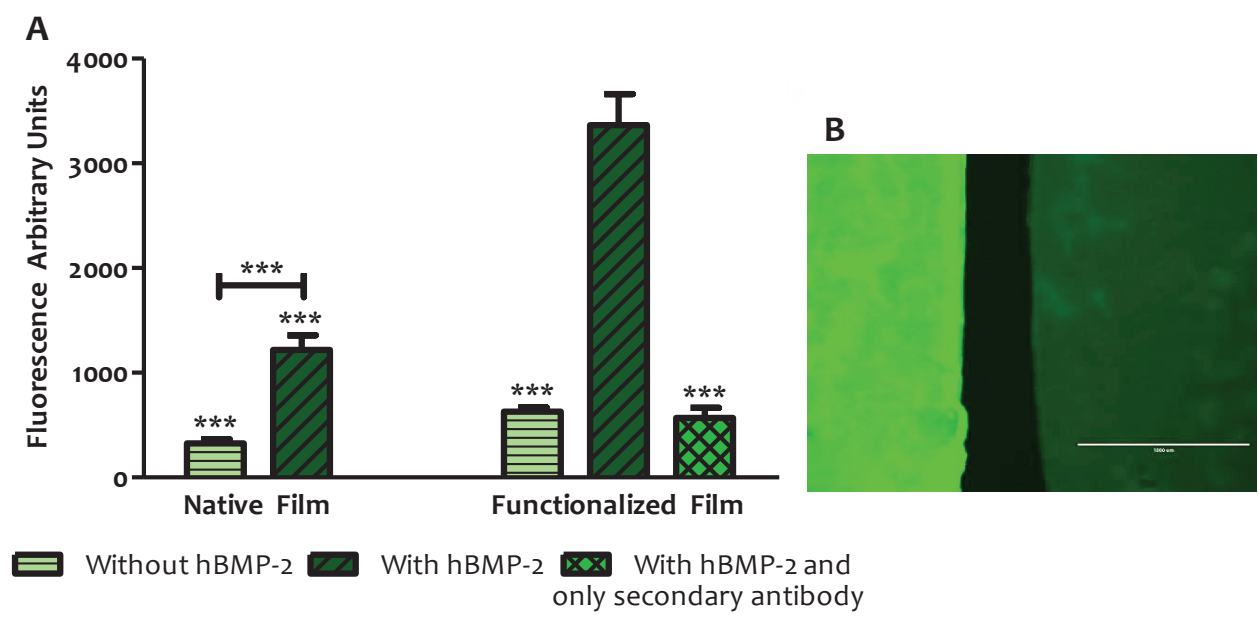

Figure 4.2. (A) Fluorescence quantification of the immunochemistry assay against hBMP-2 immobilized on the films. The data represent the mean \pm SD of three measurements per sample $(n=$ 2). $* * * p<0.0001$ (two-tailed unpaired t-test) with functionalized films incubated with hBMP-2 and immunochemistry performed with primary and secondary antibodies. (B) Fluorescence image of the functionalized film with hBMP-2 incubation (left) vs native film with hBMP-2 incubation (right) (bar: $1,000 \mu \mathrm{m})$. 


\subsubsection{Immobilized hBMP-2 retains its bioactivity}

A BMP-2 reporter cell line was used in order to confirm that the immobilized hBMP-2 retained its bioactivity. Both native and functionalized films were incubated with different amounts of hBMP-2 prior to cell seeding, and on the following day the luciferase was quantified and normalized to the amount of DNA (Figure 4.3). Our results showed that in the native films, regardless of the concentration of hBMP-2 used, no upregulation of luciferase was observed when compared to the native films without hBMP-2 incubation. In contrast, when the functionalized films were incubated with hBMP-2 before cell seeding, the hBMP-2 immobilized by the peptide retained its bioactivity, since it induced a significant increase in the amount of luciferase produced for all of the concentrations tested when compared with the condition where the functionalized films where not incubated with hBMP-2. The amounts of hBMP-2 were tested in the range of 0.5 to $250 \mathrm{ng}$, and this led to similar levels of luciferase as those produced in the functionalized films. The maximum value of luciferase produced in response to the immobilized hBMP-2 was observed when the functionalized films were incubated with $500 \mathrm{ng}$ of hBMP-2. The reported value of $10.4 \pm 1.6$ for this concentration was statistically significant when compared with all the other amounts tested, except for the case of $250 \mathrm{ng}$. The amount of $500 \mathrm{ng}$ was used for further experiments.

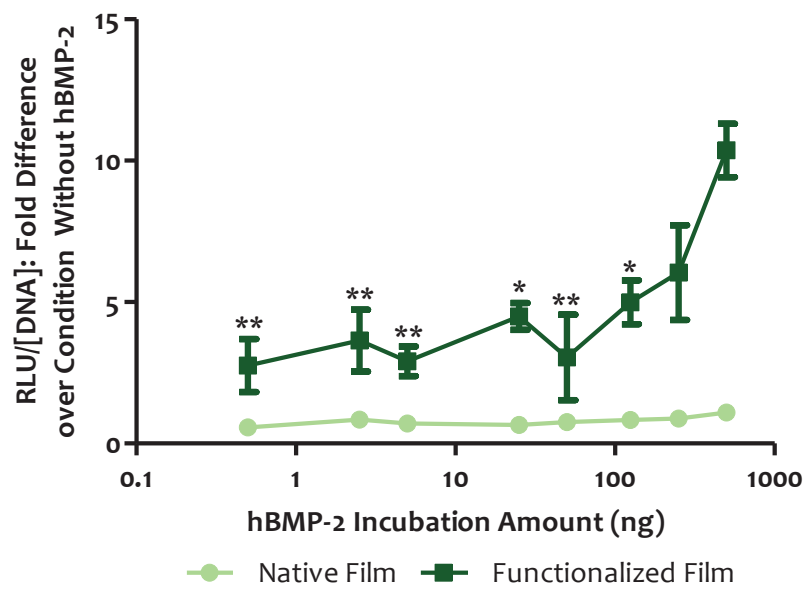

Figure 4.3. Dose-response curve of immobilized hBMP-2 on the expression of luciferase by a BMP-2 reporter cell line. Luciferase values were corrected for the amount of DNA and normalized to the condition without incubation of BMP-2 prior to cell seeding. * $p<0.05$ and $* * p<0.01$ with a one-way ANOVA with Tukey's post-hoc test for analysis between a given hBMP-2 incubation amount and the incubation amount of $500 \mathrm{ng}$ in the condition of the functionalized films. The data are represented as mean \pm SD of three samples. 
Next, we performed a further experiment with the BMP-2 reporter cell line, where the cells were cultured on top of native films, films covalently modified until the NHS/EDC step, films with a scrambled peptide (Figure S4.2) and films with a BMP-2-binding peptide. The films were incubated with a solution containing $500 \mathrm{ng}$ of hBMP-2, the cells seeded in the absence or presence of $2 \mathrm{ng} / \mathrm{mL}$ of a specific BMP-2 inhibitor (Noggin) and the amount of luciferase quantified and normalized to the amount of DNA (Figure 4.4). Our results show that the incubation of films functionalized with a scrambled peptide with hBMP-2 prior to cell seeding did not lead to an increase in the amount of luciferase produced when compared with the same films without hBMP-2 incubation. In contrast, the presence of a BMP-2-binding peptide on the films allowed the immobilization of the hBMP-2, which induced a 5.3 \pm 2.0 -fold increase in the amount of luciferase produced ( $p$ $<0.05)$. Culture of the cells in the presence of a BMP-2 inhibitor completely suppressed the luciferase activity observed in response to the immobilized hBMP-2 $(p<0.01)$.

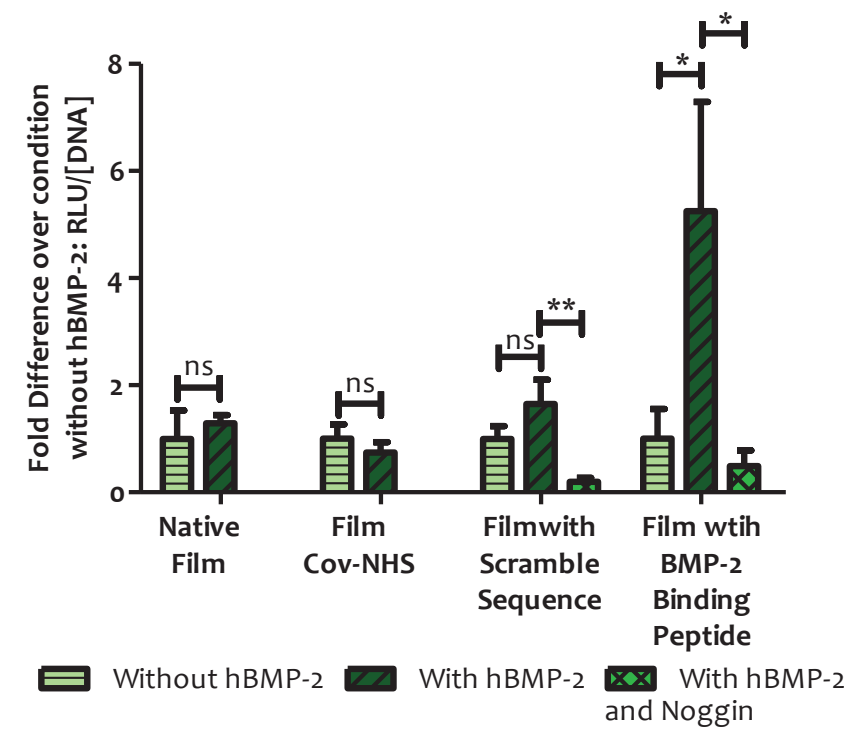

Figure 4.4. Effect of immobilized hBMP-2 on the expression of luciferase by a BMP-2 reporter cell line. Luciferase values were corrected for the amount of DNA and normalized to the condition without incubation of BMP-2 prior to cell seeding. * $p<0.05$ and ** $p<0.01$ (two-tailed unpaired t-test). The data are represented as mean \pm SD of three samples. 


\subsubsection{Synthesis of ALP in response to immobilized hBMP-2}

BMP-2 is known to direct the differentiation of $\mathrm{C}_{2} \mathrm{C}_{12}$ myoblasts into osteoblasts [22]. In order to identify the amount of immobilized hBMP-2 needed to transdifferentiate the $\mathrm{C}_{2} \mathrm{C}_{12}$ into the osteogenic lineage, a dose response study of immobilized hBMP-2 was performed (Figure 4.5). Films functionalized with a BMP-2-binding peptide were incubated with 0, 0.5, 2.5 and 5.0 $\mu \mathrm{g}$ of hBMP-2 and the amount of ALP synthesized was quantified after 7 days of culture. Our results showed that incubation of the films with $500 \mathrm{ng}$, as used in previous experiments, was insufficient to induce the osteogenic differentiation of $\mathrm{C}_{2} \mathrm{C}_{12}$. Based on this, the concentration of hBMP-2 (2.5 and 5.0 $\left.\mu \mathrm{g}\right)$ used for the pre-incubation was increased and, when doing so, a significant increase was shown in the amount of ALP produced when compared to the controls, reaching a maximum of a $12.7 \pm 2.2$-fold increase for $5.0 \mu \mathrm{g}$. This amount of BMP was then used for further experiments.

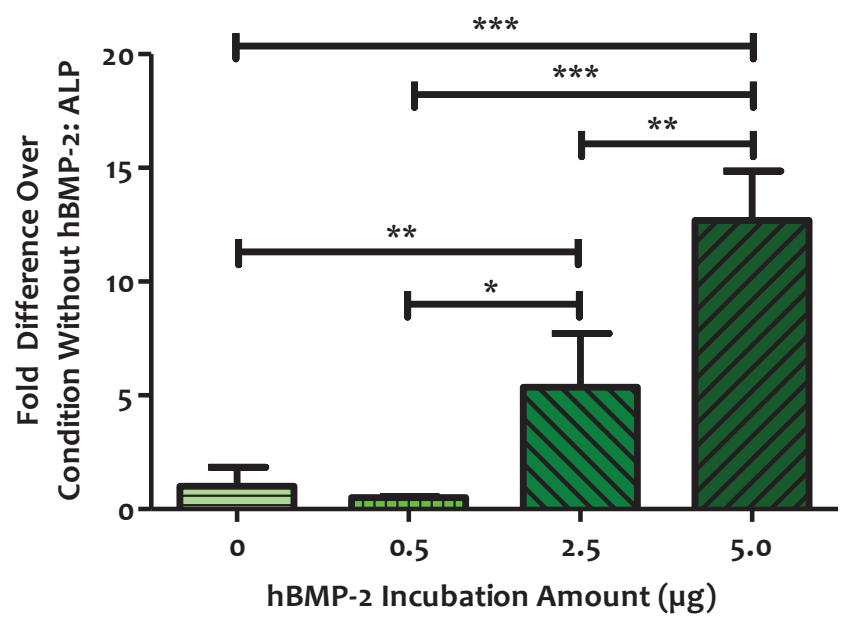

Figure 4.5. Effect of immobilized hBMP-2 on the expression of luciferase by a BMP-2 reporter cell line. Luciferase values were corrected for the amount of DNA and normalized to the condition without incubation of hBMP-2 prior to cell seeding. * $p<0.05, * * p<0.01$ and $* * * p<0.0001$ with a one-way ANOVA with Tukey's post-hoc test for analysis between all concentrations. The data are represented as mean \pm SD of two measurements per sample $(n=3)$.

Next, $\mathrm{C}_{2} \mathrm{C}_{12}$ were cultured on top of native films and films functionalized with a BMP-2binding peptide that were incubated with $5.0 \mu \mathrm{g}$ (ihBMP2) or without hBMP-2 before the cell seeding. In the case of the films without hBMP-2 incubation before cell seeding, the $\mathrm{C}_{2} \mathrm{C}_{12}$ were either cultured in the presence of $200 \mathrm{ng} / \mathrm{mL}$ (shBMP-2) or in the absence of soluble hBMP-2. Each time the medium was refreshed, new soluble hBMP-2 was added 
only for that condition, and ALP was quantified after 7 days (Figure 4.6). Our results showed that the incubation of both native and functionalized films with hBMP-2 prior to cell seeding led to an increase in the amount of ALP synthetized. However, the ALP production in response to the hBMP-2 immobilized by the BMP-2-binding peptides was 2.4 higher when compared with the response to the hBMP-2 nonspecifically adsorbed in the native films. Treatment with soluble hBMP-2 led to an even more pronounced response when compared with the condition in which the same films were incubated with hBMP-2 before cell seeding. Interestingly, there was no significant difference between the treatment with soluble hBMP-2 in the native films and the immobilized hBMP-2 in the functionalized films. Staining against ALP corroborated the results obtained from the ALP quantification.

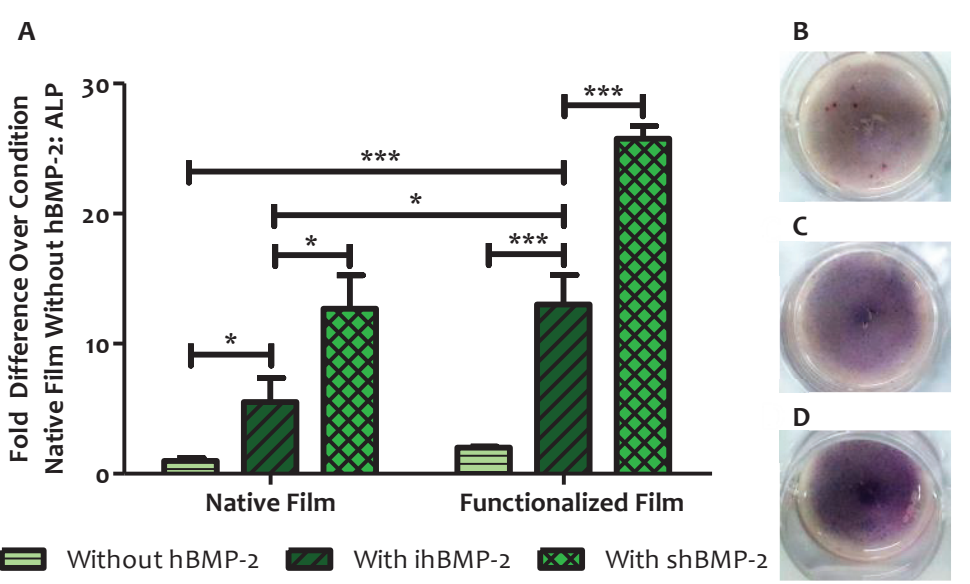

Figure 4.6. (A) Quantification of ALP synthetized in response to immobilized hBMP-2 (ihBMP-2) and soluble hBMP-2 (shBMP-2) in native and functionalized films. ${ }^{*} p<0.05,{ }^{* *} p<0.01, * * * p<0.0001$ (two-tailed unpaired t-test). The data represent the mean $\pm S D$ of two measurements per sample ( $n$ =3). (B) ALP staining in functionalized films without $h B M P-2$, (C) in functionalized films with ihBMP-2 and (D) functionalized films with shBMP-2.

\subsubsection{Capture of endogenous BMP-2 by the BMP-2-binding peptide leads to tissue response}

During the inflammation stage of tissue healing, several cytokines are produced and released. These cytokines influence several cellular processes inherent in tissue healing. We envisioned that the BMP-2-binding peptide could capture and accumulate the BMP2 released during this stage, leading to a more pronounced tissue response. Both native and functionalized PCL films were subcutaneously implanted in the back of rats, facing 
the fascia. Each animal, with a total of three animals per time point, was implanted with both native and functionalized film. At day 3 and day 7 after implantation, samples were harvested and used for immunofluorescence against BMP-2 and for histology. Only the samples from day 7 were used for immunofluorescence against BMP-2. Our fluorescence results reported a higher signal in the functionalized films in all three animals when compared with the native films after 7 days of implantation (Figure 4.7). From our histological results, the accumulation of BMP-2 in the functionalized films resulted in a more pronounced recruitment of inflammatory cells and in the appearance of blood vessel-like structures (yellow arrows) at day 3 when compared with the native films (Figure 4.8 A-D). After 7 days, further matrix deposition (pink colour) was observed around the functionalized films, with the tissue showing a higher degree of organization when compared with the native films (Figure 4.8 E-F). Overall, these results show that the BMP-2 captured by the peptide led to the recruitment of inflammatory cells and to the appearance of blood vessel-like structures after 3 days, and increased matrix deposition and tissue organization after 7 days.
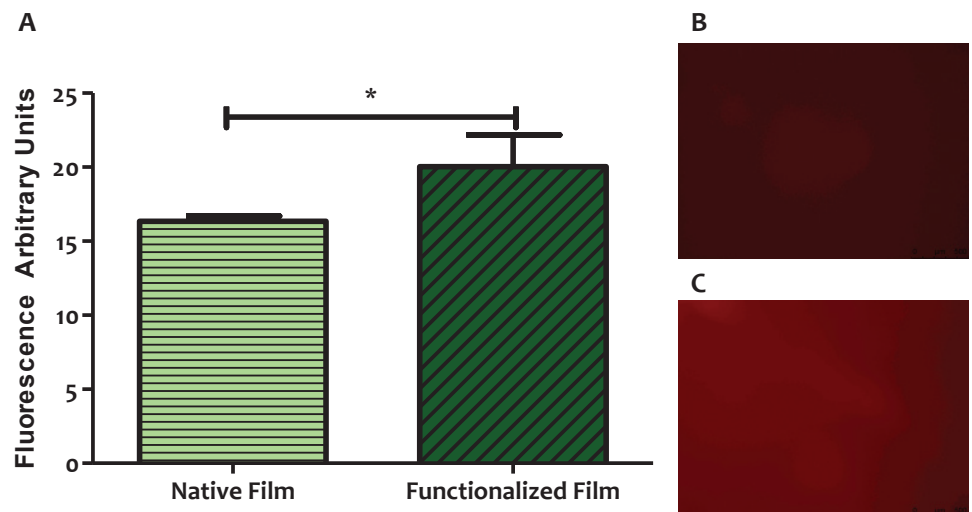

Figure 4.7. (A) Fluorescence quantification of the immunochemistry assay against BMP-2 immobilized on implanted films. The data represent mean \pm SD of five measurements per sample $(n=3)$. $p<0.05$ (two-tailed unpaired t-test). (B) Fluorescence image of the native film implanted subcutaneously on rats. (C) Fluorescence image of the film functionalized with BMP-2-binding peptide implanted subcutaneously on rats. 

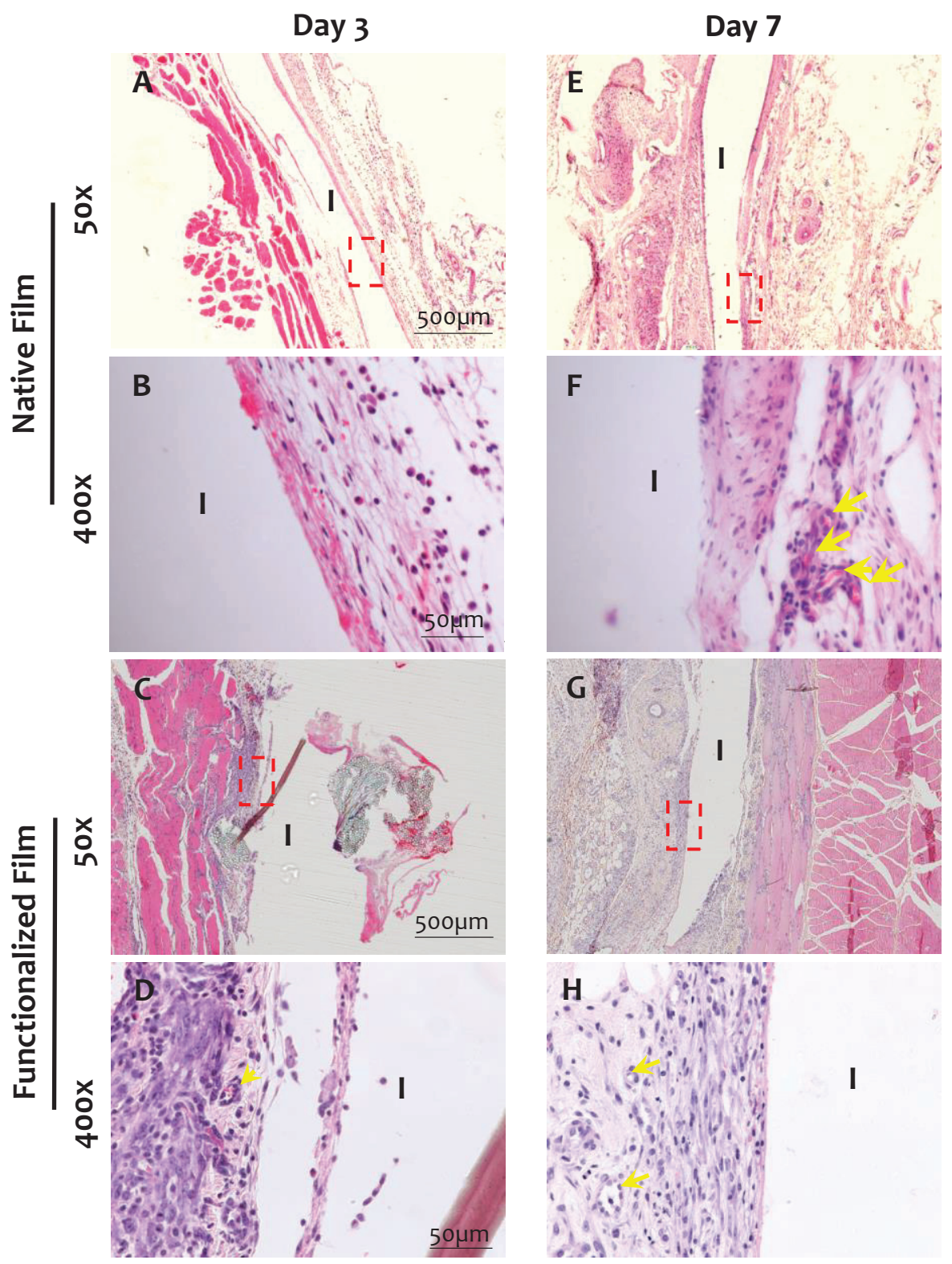

Figure 4.8. Histological evaluation of samples sections after $3(A-D)$ and $7(E-H)$ days of implantation. Haematoxylin and eosin staining on native films ( $A, B, E$ and $F)$ and functionalized films (C, G, D and H). Implant (I), blood vessels (yellow arrows), magnified area (red dashed box). 


\subsection{Discussion}

The goal of this work was to study the effect of delivering hBMP-2 via the interaction with a BMP-2-binding peptide in the activation of the BMP cascade and in osteoblast differentiation. In this investigation, synthetic BMP-2-binding peptides were covalently immobilized onto PCL films and the interaction of these peptides with hBMP-2 was confirmed using immunochemistry. The hBMP-2 captured by the peptide on the films remained active and triggered the BMP signalling pathway, while the amount of hBMP2 was sufficient to induce osteoblast differentiation of $\mathrm{C}_{2} \mathrm{C}_{12}$. Finally, implants functionalized with the BMP-2-binding peptide were subcutaneously implanted in the back of rats. These films captured the endogenous BMP-2 in contrast to control films, as could be concluded from the observed increase in the recruitment of inflammatory cells and the appearance of blood vessel-like structures after 3 days, and increased matrix deposition and tissue organization after 7 days.

BMPs are one of the most important classes of GF, playing various specific roles from early embryogenesis to the maintenance of adult homeostasis [23, 24]. During embryogenesis, BMPs are crucial for the differentiation of the mesoderm and the development of the cardiac system. Knocking out BMP-2 and BMP-4 in mice has been shown to be lethal to the embryo, while death occurred shortly after birth for BMP-1, -7 and -14 knockout mice [8]. BMPs are also involved in processes such as the regulation of cell proliferation, differentiation and apoptosis, which are crucial events during tissue remodelling and regeneration, making the use of BMPs in tissue engineering and regenerative medicine extremely attractive [25]. In fact, BMPs are one of the most powerful cytokines involved in inducing bone formation. BMPs induce osteoblast differentiation of MSCs, increase the expression of proteins found in the bone matrix such as osteocalcin, and accelerate calcium mineralization, with this latter function being translated into more pronounced bone matrix synthesis [26]. Of all the GFs known to be involved in the healing and regeneration process, only BMP-2 and BMP-7 are clinically approved by the Food and Drug Administration (FDA). Infuse ${ }^{\circledR}$ (rh-BMP-2, Medtronic) and $\mathrm{OP}-1{ }^{\circledR}$ (rh-BMP7, Olympus) are the two authorized products that make use of BMPs, both of which are used for bone therapy. Both are composed of a collagen sponge carrier that localizes the rhBMP at the site of implantation, slowly releasing the GF over time. Infuse ${ }^{\circledR}$ is clinically used in the treatment of degenerative disc diseases, while OP-1® is used for the treatment of long bone nonunion fractures and for revision posterolateral lumbar spine fusion [27, 28]. Due to the fact that the BMPs are simply adsorbed to the collagen sponge through weak interactions (i.e., electrostatic and Van der Waals forces) 
and the short-life of this GF in the body, the localization and presentation of the BMP in the implantation site for sufficient time to induce human osteogenesis is only achieved through the use of supraphysiological amounts of the GF by these two medical devices [13]. The use of such large amounts of BMP are known to be associated with several adverse effects and also raise new risks. Zara et al. (2011) showed that the use of high BMP-2 concentrations leads to the formation of cyst-like bony shells filled with adipose tissue in a rat femoral segmental defect model. In the same study, the use of high BMP2 concentrations also resulted in significant tissue inflammatory infiltrates in a rat traumatic femoral onlay model [14]. Several other studies have also reported that the "off-label” use of BMP/Infuse ${ }^{\circledR}$ has led to severe adverse effects, such as heterotopic ossification, osteolysis, infection, neurological complications and cancer [28]. These complications associated with the BMP-2 doses used in bone therapy emphasize the need to explore new strategies for the delivery of BMP-2 in order to reduce the amount needed to induce human osteogenesis. Lowering the BMP-2 dose could also potentially minimize the adverse risks associated with the supraphysiological concentrations of this GF used, making the overall treatment more efficient, safer and less expensive for the patient. Strategies that rely on stronger interactions between the BMP and the delivery vehicle can extend the localization and presentation of the GF within the implantation site by minimizing its loss through diffusion, consequently reducing the amount of GF needed. The immobilization of GFs can be achieved through covalent and non-covalent bonds to the delivery vehicle [17]. Covalent immobilization of GFs to carriers can extend the presentation of the protein to the surrounding tissue when compared with noncovalent methods [15]. However, covalent immobilization has some limitations, as the immobilized GF may lose its bioactivity due to conformational changes in the structure that may occur during the immobilization procedure [29, 30]. The role of GFs in tissue healing is highly spatially-temporally coordinated, and excess amounts of the GF could potentially lead to undesired effects. Non-covalent immobilization of GFs is a more natural way of delivery than the use of covalent bonds, since it relies on interactions involved in the immobilization of GFs in the ECM, such as electrostatic and hydrophobic interactions. ECM is known to function as an anchor matrix for GF, binding and releasing GFs and consequently concentrating their activity at the site [30]. ECM proteins, such as heparin, are known to bind different GFs and have been efficiently used as carriers for different GFs. The immobilization of synthetic peptides into materials that can trigger a cell response or have an affinity for biomolecules has emerged as new strategy in giving biological properties to implanted materials [31-33]. Saito et al. (2005) showed that the functionalization of alginate gels with a synthetic peptide corresponding to BMP-2 residues 73-92 induced the formation of ectopic bone when implanted in rat tibial bone 
defects [34]. Stupp et al. (2005) screened a phage display library of peptides composed of 7 amino acids against different GFs and identified several amino acid sequences that display an affinity for GFs, such as TGF- $\beta 1$, BMP-2 or VEGF [20]. Here, we present a noncovalent approach to specifically deliver hBMP-2 by functionalizing PCL films with a BMP2-binding peptide.

The affinity of the peptide for hBMP-2 was confirmed by immunochemistry (Figure 4.2). From our results, we can conclude that functionalization of biomaterials with the BMP2-binding peptide allows the immobilization of hBMP-2 on them through a non-covalent interaction. The incubation of native PCL films with hBMP-2 also led to an increase in the fluorescence intensity when compared with native films without hBMP-2 incubation; this can be due to unspecific adsorption of the hBMP-2 to the PCL. However, the highest fluorescent intensity observed was in the case of the functionalized films with hBMP-2 incubation, showing the advantages of incorporating a BMP-2-binding peptide onto biomaterials in order to achieve the immobilization of hBMP-2.

The affinity of the peptide towards hBMP-2 was confirmed by immunochemistry (Figure 4.2). From our results we can conclude that functionalization of biomaterials with the BMP-2-binding peptide allows the immobilization of hBMP-2 on them through a noncovalent interaction. Incubation of native PCL films with hBMP-2 also led to an increase in the fluorescence intensity when compared with native films without hBMP-2 incubation which can be due to unspecific adsorption of the hBMP-2 to the PCL. However, the highest fluorescent intensity observed was in the case of the functionalized films with hBMP-2 incubation, showing the advantages of incorporating a BMP-2-binding peptide on biomaterials in order to achieve immobilization of hBMP-2.

BMPs initiate their signalling by forming a hetero-tetrameric complex upon binding to the type I and II transmembrane receptors [35]. Immediately after interaction occurs between the BMP and the type II receptor, which is constituently active and phosphorylated, the type I receptor will be recruited and phosphorylated. Phosphorylation of the type I receptor will in turn phosphorylate and activate the receptor-mediated mother against decapentaplegic (R-SMAD), including Smad $1 / 5 / 8$. The phosphorylated Smad1/5/8 will from a complex with Smad 4 and translocate to the nucleus, where upon interaction with different expression modulators will activate the BMP target genes. In addition to the Smad dependent pathway, BMP are also known to exert their effect through the Ras/ERK/MAPK pathways [35]. In order to further investigate whether the immobilized hBMP-2 retained its bioactivity, we used a cell line stably transfected with a construct consisting of a BMP/Smad enhancer from the Id1 
promoter that was fused to a luciferase reporter gene [21]. We hypothesized that if the immobilized BMP-2 retained its bioactivity, it would activate the Id1 promoter and induce the production of luciferase. J. Almodóvar et al. (2014) created gradients of BMP-2 adsorbed on polymer films and showed that a certain surface concentration of BMP-2 was required in order to activate the signalling pathway in $\mathrm{C}_{2} \mathrm{C}_{12}$ [36]. We first studied the luciferase response of $\mathrm{C}_{2} \mathrm{C}_{12}$ that were cultured on top of films that were incubated with different amounts of hBMP-2 prior to cell seeding (Figure 4.3). In the case of the native films, no luciferase upregulation was observed for any of the amounts tested when compared with the condition without hBMP-2 incubation before cell seeding. When cultured on top of films functionalized with a BMP-2-binding peptide, $\mathrm{C}_{2} \mathrm{C}_{12}$ transfected with this luciferase construct exhibited a dose-dependent luciferase response to increasing incubation concentrations of hBMP-2 from 50 to $500 \mathrm{ng}$, reaching a maximum for the highest amount tested. Incubation of functionalized films with hBMP2 amounts lower than $50 \mathrm{ng}$ also led to an increase in the amount of luciferase produced. However, there was no significant difference in the luciferase produced in response to the amounts of hBMP-2 tested between 0.5 and $50 \mathrm{ng}$. We selected the incubation amount of $500 \mathrm{ng}$ of hBMP-2 for the following experiment, in which we included a control with films functionalized until the NHS/EDC step and with a scramble peptide sequence (Figure 4.4). Additionally, the cells were cultured either in the presence or absence of Noggin, which is a known inhibitor of the BMP signalling cascade. Again, the incubation of films lacking a BMP-2-binding peptide with $500 \mathrm{ng}$ of hBMP-2 before cell seeding did not lead to an upregulation of the amount of luciferase produced. This can be explained by the fact that these films lack an affinity for hBMP-2; therefore, there is no or insufficient hBMP-2 on the surface to trigger the BMP signalling pathway and consequently luciferase production by these cells. In contrast, when the films functionalized with a BMP-2-binding peptide were incubated with BMP-2 prior to cell seeding, an increase in luciferase activity was observed. When the cells were seeded in the presence of Noggin, which is known to bind to BMP and therefore block its interaction with the cell membrane receptors, the luciferase produced in response to the immobilized hBMP-2 was abolished [37]. These results show that the hBMP-2 captured by the BMP-2-binding peptide remains bioactive and activates the BMP signalling cascade by the Smad dependent pathway.

BMP-2 is known to induce the differentiation of $\mathrm{C}_{2} \mathrm{C}_{12}$ into the osteogenic lineage, with ALP being one of the characteristic markers of osteoblast lineage cells [22]. Next, we studied whether the hBMP-2 immobilized via the interaction with the BMP-2-binding peptide was able to induce the differentiation of $\mathrm{C}_{2} \mathrm{C}_{12}$ into osteoblasts. Although the 
loading amount of $500 \mathrm{ng}$ on the functionalized films was able to activate the BMP signalling pathway on $\mathrm{C}_{2} \mathrm{C} 12$, this amount was insufficient to direct their differentiation into osteoblasts (Figure 4.5). Based on our ALP dose response on functionalized films, we concluded that in order to direct the differentiation of $\mathrm{C}_{2} \mathrm{C}_{12}$ into osteoblasts, the functionalized films were required to be loaded with amounts higher than $500 \mathrm{ng}$ of hBMP-2, with the loading amount of $5.0 \mu \mathrm{g}$ selected for further experiments (Figure 4.5). Our results show that preincubation of functionalized films with $5.0 \mu \mathrm{g}$ of hBMP-2 before cell seeding led to a 2.4-fold higher production of ALP when compared with the native films that were also preloaded with hBMP-2 before cell seeding (Figure 4.6). The difference in ALP production can be explained by a higher surface concentration of hBMP-2 in the functionalized films, and due to a longer presentation and availability of the hBMP-2 due to the affinity interaction with the BMP-2-binding peptide. This highlights the advantages of delivering hBMP-2 to cells via the interaction with the BMP2-binding peptide over non-specifically adsorbing the GF to the biomaterial. There was no difference in ALP production between the soluble treatment in the native films and the functionalized films pre-loaded with hBMP-2. Interestingly, treatment with soluble hBMP-2 in the functionalized films resulted in a two-fold increase in ALP production when compared with the soluble treatment in the native films. One hypothesis for this is that the part of the hBMP-2 added to the cells interacts with the receptors of the cells while the remaining hBMP-2 are captured by the BMP-2 binding, making the cells sense both soluble and immobilized hBMP-2. Most likely the hBMP-2 that interact with the receptors trigger the signalling cascade, while the immobilized hBMP-2 maintain the active pathway for longer periods.

We envisioned that once implanted in vivo, the BMP-2-binding peptide would capture endogenous BMP-2 and induce a tissue response. When implanted subcutaneously onto the backs of rats, functionalized films led to a higher accumulation of BMP-2 at the implant when compared with native films (Figure 4.7). This accumulation resulted in the increased recruitment of inflammatory cells and the appearance of blood vessel-like structures after 3 days around the functionalized implant (Figure 4.8 A-D). Jian et al. showed that silk scaffolds loaded with BMP-2 subcutaneously implanted in rabbit skull defects led to the recruitment and homing of mesenchymal stem cells when compared with the negative control [38]. In the same work, angiogenesis was observed in the scaffolds loaded with BMP-2 in contrast to the negative controls. After 7 days, increased matrix deposition was observed around the functionalized implant, which also presented a highly organized tissue when compared with the native implant that showed nearly no organization (Figure $4.8 \mathrm{E}-\mathrm{F}$ ). Due to these promising results, which showed 
the appearance of blood vessel-like structures and increased matrix deposition around the functionalized implant, which is extremely important in bone tissue as this tissue is mainly composed of hydroxyapatite and collagen with a dense vascular network, further in vivo studies will be conducted in the future. In this way, we expect to gain a better understanding of the potential of functionalizing biomaterials with a BMP-2-binding peptide in order to deliver or capture BMP-2 in bone defects.

Taken together, the results show the potential benefits of immobilizing hBMP-2 on biomaterials via the interaction with a BMP-2-binding peptide. Synthetic peptides have several advantages over conventional immobilization systems, since they can be easily and rapidly synthetized through chemical peptide synthesis. Synthetic peptides are a promising tool in the improvement and design of medical devices, giving the ability to immobilize and deliver GFs or to capture endogenous GFs. Ward et al. (2010) showed that it is possible to functionalize collagen matrices by reacting the carboxylic end of the collagen molecule with NHS/EDC and then reacting these with free amines of biomolecules [39]. Functionalization of the current hBMP-2-based therapy devices with the hBMP-2-binding peptide could prolong the presentation of the GF to the surrounding tissue and therefore reduce the amount of hBMP-2 required for the treatment. This could potentially minimize the risks associated with the use of high doses of hBMP-2 and significantly reduce the treatment costs.

\subsection{Conclusions}

In this work, we describe a strategy to immobilize hBMP-2 via the interaction with a BMP2-binding peptide. The peptide was covalently immobilized in $\mathrm{PCL}$ and its affinity towards the GF confirmed by immunochemistry. Our in vitro studies showed that the immobilized hBMP-2 activated the BMP signalling cascade though the Smad-dependent pathway and supported osteogenic differentiation of $\mathrm{C}_{2} \mathrm{C}_{12}$. In fact, osteogenic differentiation in response to hBMP-2 immobilized by the peptide was significantly higher when compared with the response to the hBMP-2 that was only adsorbed to the native film. In vivo results showed a higher accumulation of BMP-2 in the implant functionalized with the BMP-2binding peptide, which led to a higher recruitment of inflammatory cells, vascularization and matrix deposition. Our findings highlight the potential of using this peptide sequence in the design of improved medical devices in order to deliver and/or capture hBMP-2 to/from the surrounding tissue. This development would allow a longer retention time of the $\mathrm{GF}$ in the biomaterial, which could potentially reduce the amount of GF required in order to successfully repair the damaged tissue. 


\subsection{Acknowledgments}

We acknowledge Smith\&Nephew for financial support and Dr. Bruma Fu and Angel Lee from the University of Hong Kong for their collaboration in the in vivo experiments.

\subsection{References}

1. Barrientos S, et al., Growth factors and cytokines in wound healing. Wound Repair Regen, 2008. 16(5): p. 585-601.

2. Miyazawa K, et al., Two major Smad pathways in TGF-beta superfamily signalling. Genes Cells, 2002. 7(12): p. 1191-204.

3. Carreira AC, et al., Bone Morphogenetic Proteins: structure, biological function and therapeutic applications. Arch Biochem Biophys, 2014. 561: p. 64-73.

4. Garimella R, et al., Expression and synthesis of bone morphogenetic proteins by osteoclasts: a possible path to anabolic bone remodeling. J Histochem Cytochem, 2008. 56(6): p. 569-77.

5. Lu HF, et al., Mechanical loading induced expression of bone morphogenetic protein-2, alkaline phosphatase activity, and collagen synthesis in osteoblastic MC3T3-E1 cells. Chin Med J (Engl), 2012. 125(22): p. 4093-7.

6. Kalén A, et al., The content of bone morphogenetic proteins in platelets varies greatly between different platelet donors. Biochem Biophys Res Commun, 2008. 375(2): p. 261-4.

7. McCullough KA, et al., Immunohistochemical localization of bone morphogenetic proteins (BMPs) 2, 4, 6, and 7 during induced heterotopic bone formation. J Orthop Res, 2007. 25(4): p. 465-72.

8. Wang RN, et al., Bone Morphogenetic Protein (BMP) signaling in development and human diseases. Genes Dis, 2014. 1(1): p. 87-105.

9. Sykaras $\mathrm{N}$ and Opperman LA, Bone morphogenetic proteins (BMPs): how do they function and what can they offer the clinician? J Oral Sci, 2003. 45(2): p. 57-73.

10. Chen D, et al., Differential roles for bone morphogenetic protein (BMP) receptor type IB and IA in differentiation and specification of mesenchymal precursor cells to osteoblast and adipocyte lineages. J Cell Biol, 1998. 142(1): p. 295-305.

11. Gautschi OP, Frey SP, and Zellweger R, 2007. ANZ J Surg, Bone morphogenetic proteins in clinical applications. 77(8): p. 626-31.

12. Lissenberg-Thunnissen SN, et al., Use and efficacy of bone morphogenetic proteins in fracture healing. Int Orthop, 2011. 35(9): p. 1271-80.

13. King WJ and Krebsbach PH, Growth factor delivery: how surface interactions modulate release in vitro and in vivo. Adv Drug Deliv Rev, 2012. 64(12): p. 1239-56.

14. Zara JN, et al., High Doses of Bone Morphogenetic Protein 2 Induce Structurally Abnormal Bone and Inflammation In Vivo. Tissue Eng Part A, 2011. 17((9-10)): p. 1389-1399.

15. Masters KS, Covalent growth factor immobilization strategies for tissue repair and regeneration. Macromol Biosci, 2011. 11(9): p. 1149-63.

16. Pohl TL, et al., Surface immobilization of bone morphogenetic protein 2 via a selfassembled monolayer formation induces cell differentiation. Acta Biomater, 2012. 8(2): p. $772-80$. 
17. Lee K, Silva EA, and Mooney DJ, Growth factor delivery-based tissue engineering: general approaches and a review of recent developments. J R Soc Interface, 2011. 8(55): p. 153-70.

18. Martino MM, et al., Heparin-binding domain of fibrin(ogen) binds growth factors and promotes tissue repair when incorporated within a synthetic matrix. PNAS, 2013. 110(12): p. 4563-8.

19. Yang HS, et al., Comparison between heparin-conjugated fibrin and collagen sponge as bone morphogenetic protein-2 carriers for bone regeneration. Exp Mol Med, 2012. 44(5): p. 350-5.

20. Stupp SI, et al., Self-assembling peptide amphiphiles and related methods for growth factor delivery N. University, Editor 2009.

21. Zilberberg $\mathrm{L}$, et al., A rapid and sensitive bioassay to measure bone morphogenetic protein activity. BMC Cell Biol, 2007. 8(41).

22. Katagiri T, et al., Bone morphogenetic protein-2 converts the differentiation pathway of C2C12 myoblasts into the osteoblast lineage. J Cell Biol, 1994. 127(6 Pt 1): p. 1755-66.

23. Kishigami S and Mishina Y, BMP signaling and early embryonic patterning. Cytokine Growth Factor Rev, 2005. 16(3): p. 265-78.

24. Bandyopadhyay A, Yadav PS, and Prashar P, BMP signaling in development and diseases: a pharmacological perspective. Biochem Pharmacol, 2013. 85(7): p. 857-64.

25. Zhang J and Li L, BMP signaling and stem cell regulation. Dev Biol, 2005. 284(1): p. 1-11.

26. Chen G, Deng C, and Li YP, TGF-B and BMP signaling in osteoblast differentiation and bone formation. Int J Biol Sci, 2012. 8(2): p. 272-88.

27. Delawi D, et al., OP-1 Compared with Iliac Crest Autograft in Instrumented Posterolateral Fusion: A Randomized, Multicenter Non-Inferiority Trial. J Bone Joint Surg Am, 2016. 98(6): p. 441-8.

28. Epstein NE, Complications due to the use of BMP/INFUSE in spine surgery: The evidence continues to mount. Surg Neurol Int, 2013. 4(5): p. S343-352.

29. Bruning JB, et al., Coupling of receptor conformation and ligand orientation determine graded activity. Nat Chem Biol, 2010. 6(11): p. 837-43.

30. Schultz GS and Wysocki A, Interactions between extracellular matrix and growth factors in wound healing. Wound Repair Regen, 2009. 17(2): p. 153-62.

31. Collier JH and Segura T, Evolving the use of peptides as biomaterials components. Biomaterials, 2011. 32(18): p. 4198-4204.

32. Chan KH, Zhuo S, and Ni M, Natural and synthetic peptide-based biomaterials for bone tissue engineering. Biomaterials, 2013. 1(1).

33. Lee JY, et al., Bioactive peptide-modified biomaterials for bone regeneration. Curr Pharm Des, 2011. 17(25): p. 2663-76.

34. Saito A, et al., Accelerated bone repair with the use of a synthetic BMP-2-derived peptide and bone-marrow stromal cells. J Biomed Mater Res A, 2005. 72(1): p. 77-82.

35. Nohe A, et al., The mode of bone morphogenetic protein (BMP) receptor oligomerization determines different BMP-2 signaling pathways. J Biol Chem, 2002. 277(7): p. 5330-8.

36. Almodóvar J, et al., Spatial patterning of BMP-2 and BMP-7 on biopolymeric films and the guidance of muscle cell fate. Biomaterials, 2014. 35(13): p. 3975-85.

37. Groppe J, et al., Structural basis of BMP signaling inhibition by Noggin, a novel twelvemembered cystine knot protein. J Bone Joint Surg Am, 2003. 85-A(3:52-8).

38. Zhang $\mathrm{W}$, et al., VEGF and BMP-2 promote bone regeneration by facilitating bone marrow stem cell homing and differentiation. Eur Cell Mater, 2014. 15(27): p. 1-12. 
Chapter 4 Immobilization of hBMP-2 on polymers directs cell fate

39. Ward J, et al., Amine Functionalization of Collagen Matrices with Multifunctional Polyethylene Glycol Systems. Biomacromolecules, 2010. 11(11): p. 3093-3101. 


\subsection{Supporting Information}

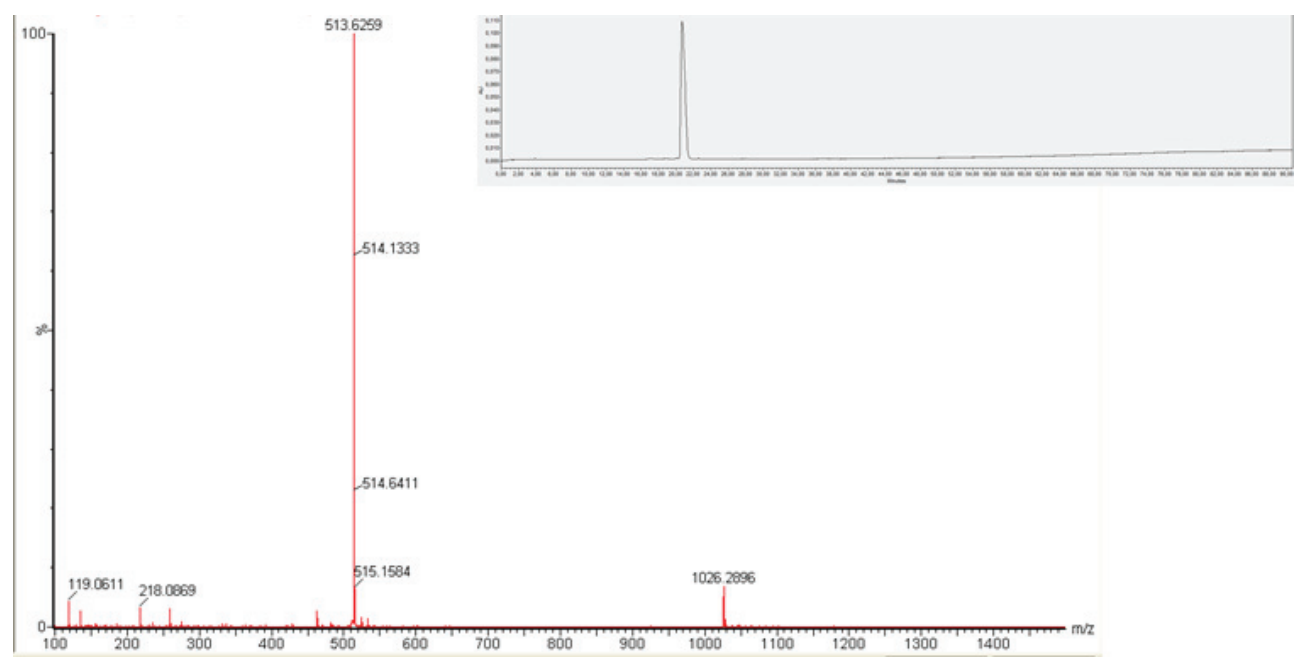

Figure S4.1. Mass spectroscopy and analytical HPLC of the BMP-2-binding peptide after purification.

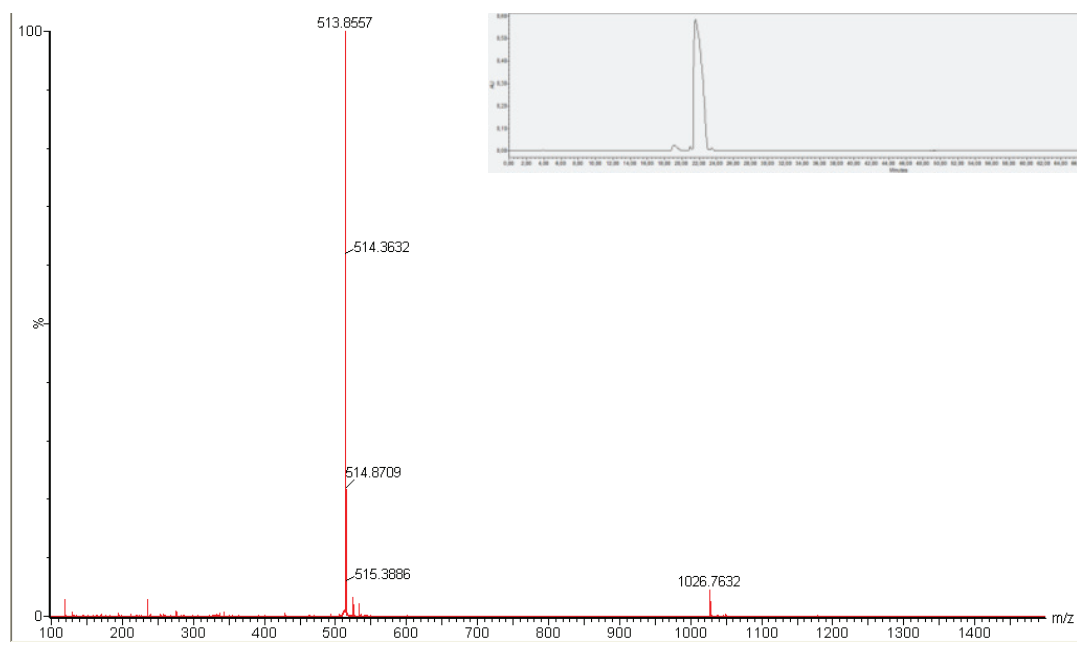

Figure S4.2. Mass spectroscopy and analytical HPLC of the BMP-scramble peptide after purification. 


\section{Chapter 5}

\section{Improvement of medical devices with VEGF-binding peptides in order to induce vascularization in orthopaedic injuries}

Adequate vascularization remains one of the biggest challenges in tissue engineering. This is of particular importance in orthopaedic injuries, where tissues such as tendons/ligaments $(T / L)$, cartilage and meniscus are poorly vascularized and therefore have a limited healing capacity. Vascularization can be induced by delivering angiogenic growth factors (GFs) such as vascular endothelial growth factor (VEGF). In this chapter, a synthetic peptide that binds VEGF was covalently attached to suitable chemically-modified polycaprolactone (PCL) films. In vitro studies showed that the immobilized VEGF retained its bioactivity and induced the survival of endothelial cells. In vivo experiments in rats showed that $P C L$ implants functionalized with the VEGF-binding peptide led to an accumulation of endogenous VEGF in the implant when compared with the (negative) control films lacking the VEGF-binding peptide. This VEGF accumulation resulted in a higher cellularity and in the appearance of blood vessel-like structures around the implant 3 days after implantation. Seven days after implantation the tissue around the functionalized implant clearly had a higher degree of organization when compared with the negative control. Subsequently, medical devices that are currently in use in orthopaedic surgeries such as Poly-tape, Chondro-Gide and Collagen Meniscus Implant (CMI) were chemically modified to react with the VEGF-binding peptide, therefore allowing for the successful immobilization of VEGF onto these medical devices. We envision that these VEGF-binding devices can capture the patient's VEGF and consequently trigger vascularization and promote healing. 


\subsection{Introduction}

One of the current challenges in tissue engineering is the proper vascularization of tissue constructs grown in vitro, as this will ultimately affect their integration once implanted in vivo $[1,2]$. The inadequate vascularization of tissue will lead to mass transfer limitations that will affect the diffusion of oxygen, nutrients and metabolic waste of the cells with the surroundings and which will consequently compromise cell viability and the overall function of the tissue $[1,3]$. It is well established that the vascular organization of tissue will ultimately affect its healing capacity [4]. Therefore, avascular tissues such as cartilage and tendons have very low self-healing capacities and consequently, even with surgical intervention, their original biomechanical properties are seldom restored $[5,6]$. Due to the extreme importance of vascularization in the success of tissue engineering, several approaches have been explored in order to induce and control vascularization [7]. Current approaches can be classified into different categories, such as cell-based techniques, bioreactor designs, the use of microfluidic techniques, modular assembly, in vivo systems and scaffold functionalization [1]. The functionalization of scaffolds with GFs, bioactive peptides or extracellular matrix (ECM) components can induce angiogenesis and vascularization [8-10]. The functionalization of scaffolds in order to slowly release GFs such as VEGF and fibroblast growth factor (FGF) is known to enhance angiogenesis [11]. Members of the VEGF family are key players in the vascular development of the embryo and the formation of blood vessels in adults [12]. This family of GFs have a variety of functions, including proliferation, survival, invasion and migration, that are crucial for the development and maintenance of blood vessels [13]. Despite their role in the vascular system, VEGF family members are also involved in other processes, such as bone formation, haematopoiesis, wound healing and blood vessel development [14]. Radisic et al. (2008) showed that the covalent functionalization of collagen scaffolds with VEGF promotes the invasion and proliferation of endothelial cells (ECs) onto the scaffold [15]. Another study showed that the covalent immobilization of VEGF in a porous collagen scaffold promotes the proliferation of endothelial and bone marrow cells seeded onto the scaffold. The same functionalized scaffolds have a greater blood vessel density than the negative controls when used to replace a full right ventricular free wall defect in rat hearts [16]. However, the covalent immobilization of GFs may result in a loss of bioactivity due to conformational changes of the protein during the immobilization strategy. Hu et al. (2012) showed that even with a similar surface density of immobilized VEGF, the bioactivity of covalently immobilized VEGF is significantly lower than the heparin-bound VEGF [17]. Another strategy used to promote 
angiogenesis is to functionalize biomaterials with peptides that mimic the activity of the GF. Webber et al. (2011) showed that peptide amphiphiles displaying the VEGF mimetic peptide KLTWQELYQLKYKGI triggered the phosphorylation of VEGF receptors and promoted the proliferation, survival and migration of ECs in vitro. When used in a mouse hind-limb ischemia model, the peptide amphiphiles displaying the VEGF mimetic peptide led to a more efficient recovery due to the enhanced angiogenesis resulting from the activity of the peptide [18]. In order to avoid a loss of VEGF bioactivity, we chose a previously reported synthetic VEGF-binding peptide in order to achieve the immobilization of VEGF onto scaffolds [19]. Synthetic peptides have several advantages over heparin, such as an easy, standard and biological-free production. They can be designed according to the chemical strategy used to covalently immobilize them. Additionally, chemical modifications can be easily added to the $\mathrm{N}$ and $\mathrm{C}$ termini of the synthetic peptides in order to improve their stability. Here, we present for the first time a non-covalent approach, based on a VEGF-binding peptide, to specifically immobilize and deliver human VEGF (hVEGF). By functionalizing PCL films with a VEGF-binding peptide, we investigated the bioactivity of the immobilized VEGF in vitro. The functionalized films were implanted subcutaneously into rats and the amount of captured endogenous VEGF and tissue response around the implant was evaluated. This hVEGF immobilization strategy was translated to medical devices that were functionalized with the VEGF-binding peptide, endowing them with the potential to work as delivery or capture systems for VEGF. We envision that functionalizing these medical devices with the VEGF-binding peptide would allow them to capture endogenous VEGF from the patient and, consequently, to induce blood vessel formation which would ultimately lead to the enhancement of the overall healing process. 


\subsection{Materials and methods}

\subsubsection{Materials}

$\mathrm{N}, \mathrm{N}, \mathrm{N}^{\prime}, \mathrm{N}^{\prime}$-Tetramethyl-O-(1H-benzotriazol-1-yl)uronium hexafluorophosphate (HBTU) was obtained from MultiSynTech. Chloroform and 1-methyl-2-pyrrolidinone (NMP) were purchased from WR Chemicals. $\mathrm{NaOH}$ was obtained from Riedel-de Haën. All other reagents or products were purchased from Sigma-Aldrich unless noted otherwise.

\subsubsection{Peptide synthesis and purification}

Peptides were synthetized using Fmoc-solid phase peptide synthesis in a Syro II MultiSynTech automated peptide synthesizer. The VEGF-binding peptide with the sequence KGSWWAPFH was prepared on Fmoc-Rink 4-methylbenzhydrylamine (MBHA) resin (MultiSynTech $\mathrm{GmBH}, 50 \mathrm{mg}$ scale, substitution $0.52 \mathrm{mmol} / \mathrm{g}$ ), using $0.26 \mathrm{M}$ of HBTU, $0.52 \mathrm{M}$ of N,N-diisopropylethylamine (DIPEA), $2 \mathrm{M}$ of piperidine and $0.29 \mathrm{M}$ of each amino acid. The $\mathrm{N}$-terminus of the final peptide sequence was acetylated manually in $16 \%$ ( $\mathrm{v} / \mathrm{v})$ acetic anhydride, 30\% ( $\mathrm{v} / \mathrm{v})$ DIPEA and 54\% (v/v) NMP for one hour at room temperature. The peptide was cleaved from the resin and the amino acid side groups were deprotected using $95 \%(\mathrm{v} / \mathrm{v})$ trifluoroacetic acid, $2.5 \%(\mathrm{v} / \mathrm{v})$ triisopropylsilane and $2.5 \%(\mathrm{v} / \mathrm{v})$ milliQ water. The peptide was then collected by precipitation in cold diethyl ether and the organic solvents were removed in a rotatory evaporator. The peptide was redissolved in milliQ water and lyophilized overnight. The resulting product was purified using standard preparative HPLC methods. MS (ESI): $\mathrm{m} / \mathrm{z}=1156.4[\mathrm{M}+\mathrm{H}]+$ (calculated 1156.3 for $\mathrm{C}_{58} \mathrm{H}_{73} \mathrm{~N}_{15} \mathrm{O}_{11}$ ) for KGSWWAPFH.

\subsubsection{Preparation of PCL films displaying VEGF-binding peptides}

A $12.5 \%(w / v)$ solution of PCL in chloroform was prepared and homogenized by sonication. When the solution was completely homogeneous, $\mathrm{PCL}$ films were prepared by casting in a petri dish, pre-silanized with a PFDTS $(1 \mathrm{H}, 1 \mathrm{H}, 2 \mathrm{H}, 2 \mathrm{H}$ perfluorodecyltrichlorosilane, $\geq 97 \%, A B C R \mathrm{GmbH}$ ) anti-sticky layer. Upon solvent evaporation, the polymer was melted and allowed to again solidify. The polymer was then cut into circular films with a diameter of $21 \mathrm{~mm}$ in order to fit inside the wells of a 12-well plate. The individual circular films were extensively washed with demi-water and milliQ water and dried with a $\mathrm{N}_{2}$ stream. The dried films were exposed to oxygen plasma for $5 \mathrm{~min}$ (at an oxygen pressure of $1.0 \mathrm{bar}$, a vacuum pressure of $200 \mathrm{mbar}$ and a current 
of $40 \mathrm{~A}$ ) and subsequently immersed in a $1 \mathrm{M} \mathrm{NaOH}$ solution for one hour with gentle agitation. PCL films were then washed and dried as mentioned above, and incubated with a solution of $50 \mathrm{mM}$ 1:1 NHS/EDC in MES buffer for one hour with agitation. PCL films were again washed and dried as mentioned above and were incubated with $1 \mathrm{mM}$ of the peptide in phosphate buffered saline (PBS) for 4 hours with agitation. Films were then extensively washed with PBS and sterilized by incubating the films overnight in a solution of $10 \%$ penicillin/streptomycin (Life Technologies) in PBS prior to cell seeding.

\subsubsection{Water contact angle measurements}

The wettability of the PCL films was determined by a drop contact angle system (Krüss Contact Angle Measuring System G10). The contact angle was measured and calculated using Drop Analysis software. All reported contact angles are the average of $n=5$ measurements. MilliQ water was used to measure the contact angle of the films.

\subsubsection{VEGF binding and immunofluorescence}

Samples were incubated with $1 \mu \mathrm{g} / \mathrm{mL}$ of VEGF (PeproTech) in $0.5 \%(\mathrm{v} / \mathrm{v})$ PBS tween-20 (PBST) for one hour with gentle agitation. The films were then washed three times for 10 min with $0.5 \%(v / v)$ PBST and then with PBS for a further $10 \mathrm{~min}$. Next, the films were blocked for one hour with PBS containing $1 \%(\mathrm{w} / \mathrm{v})$ bovine serum albumin (BSA) and subsequently washed as described above. Afterwards, the films were incubated with a primary antibody ( $2 \mu \mathrm{g} / \mathrm{mL}$; rabbit polyclonal anti-human VEGF, PeproTech) in the blocking solution during one hour with agitation. The films were washed as mentioned above and then incubated with a secondary antibody $(8 \mu \mathrm{g} / \mathrm{mL}$; goat anti-rabbit Alexa Fluor 594, Invitrogen) in PBS containing 1\% w/v BSA for one hour with gentle agitation. Before image acquisition, the films were washed three times for 10 min with $1 \mathrm{mM} \mathrm{PBST}$, rinsed three times with PBS and dried under a N2 stream. Fluorescence intensity was quantified using ImageJ. For cell experiments, the sterile PCL films were washed three times with PBS and incubated with $0.5 \mu$ g of VEGF in $0.5 \%(v / v)$ PBST for one hour with gentle agitation. Subsequently, the films were extensively washed with PBST (0.5\%) and PBS to remove any traces of the washing buffer prior to cell seeding.

\subsubsection{Cell culture}

Human umbilical vein endothelial cells (HUVECs) were seeded at 5,000 cell/ $\mathrm{cm}^{2}$ and expanded in Endothelial Growth Medium-2 supplemented with a BulletKit (EGMTM-2 BulletKit ${ }^{\mathrm{TM}}$, Lonza). Cells were grown at $37^{\circ} \mathrm{C}$ in a humid atmosphere with $5 \% \mathrm{CO}_{2}$. The 
medium was refreshed twice per week and cells were used for further sub-culturing or cryopreservation on reaching near confluence.

\subsubsection{Cell survival}

HUVECs at passage 4 were used for experiments. The medium was removed and the cells washed with PBS. Next, the cells were incubated with $0.5 \%$ trypsin until they detached. Once detached, fresh medium was added to neutralize the trypsin and the cells were counted. Cell were seeded on films at 25,000 cells $/ \mathrm{cm}^{2}$ in Endothelial Basal Medium (EBM-2) supplemented with $2 \%$ Fetal Bovine Serum (FBS) and gentamicin/amphotericin$B(G A-1000)$ and allowed to attach overnight at $37^{\circ} \mathrm{C}$ in a $5 \% \mathrm{CO}_{2}$ incubator. The medium was changed the following day to a medium without FBS and the cells were incubated for an additional period of 24 hours. After 24 hours, the cells were lysed and the DNA content was quantified by CyQUANT cell proliferation assay (Invitrogen), following the manufacturer's instructions.

\subsubsection{Subcutaneous implantation mouse model}

All of the animal experiments were approved by the animal research ethics committee of the Chinese University of Hong Kong. Eight 12-week-old Sprague Dawley male rats were used in this study. The rats were anesthetized by the intraperitoneal injection of $10 \%$ ketamine $/ 2 \%$ xylazine (Kethalar, $0.3 \mathrm{ml}: 0.2 \mathrm{ml}$ ), and sedation was maintained by the intramuscular injection of $10 \%$ ketamine (Sigma Chemical CO, St. Louis, MO). Subcutaneous implantation of PCL was performed as follows: briefly, once the animals were anesthetized, shaved and washed, two incisions were made and native PCL and PCL functionalized with a VEGF-binding peptide were inserted into the pockets and fixed to the fascia; the skin wound was then closed using sutures. At day 3 and day 7 post implantation, the animals were sacrificed and the samples were harvested. Samples from the subcutaneous rat model were harvested at day 7 post implantation and rinsed with PBS. Harvested samples were fixed with 10\% buffered formalin for 10 min and further permeabilized with PBST for 15 min. Samples were then washed with PBS and blocked with $1 \%(\mathrm{w} / \mathrm{v})$ BSA for 1 hour in a shaker. Next, the samples were washed three times for 10 min with PBST and incubated with a dilution of 1:100 of the primary antibody (mouse polyclonal anti VEGF, sc-7269, Santa Cruz Biotechnology) overnight at $4^{\circ} \mathrm{C}$. After primary antibody incubation, samples were washed with PBST and incubated with a dilution of 1:100 of the secondary antibody (goat anti mouse IgG-FITC, sC-2010, Santa Cruz 
Biotechnology) for one hour at room temperature. Samples were washed with PBST before imaging.

\subsubsection{Histology}

At day 3 and day 7 post implantation, PCL implants and the surrounding tissues were harvested and rinsed with PBS. Harvested samples were fixed with $10 \%$ buffered formalin overnight and embedded in paraffin. Subsequently, $5 \mu \mathrm{m}$-thick paraffin sections along the transverse plane were collected. Haematoxylin and eosin (H\&E) stained sections were examined under light microscopy (Leica Microsystems, Wetzlar, Germany).

\subsubsection{Experiments with medical devices}

Poly-tape was functionalized with a VEGF-binding peptide with the same protocol used to functionalize PCL films. Both Chondro-Gide and Collagen Meniscus Implant (CMI) were incubated with $50 \mathrm{mM}$ of NHS/EDC for 2 hours. After 2 hours, the devices were washed several times with PBS and incubated with $1 \mathrm{mM}$ solution of VEGF-binding peptide in PBS for a further 2 hours. Incubation with hVEGF, immunofluorescence staining and fluorescence quantification was performed as mentioned above for the $\mathrm{PCL}$ films.

In the case of the gradient experiments, a layer of solution containing $1 \mu \mathrm{g} / \mathrm{mL}$ of hVEGF was placed on top of a glass slide. The CMIs were placed on top of the solution, with the inner side in direct contact with the solution. Staining against immobilized hVEGF was performed as described above. For imaging, an automated fluorescence microscope (BD pathway) was used and several pictures were taken along the sample with an overlap of $10 \%$ between them. Pictures were mounted as a single picture using the plugin Stitching from ImageJ, and the fluorescence was quantified along the picture also using ImageJ.

\subsubsection{Statistical analysis}

The data were analysed using a Student's paired t-test, a one-way analysis of variance followed by a Tukey's multiple comparison test $(p<0.05)$ or a two-way analysis of variance. The values represent the mean and standard deviation of three independent measurements. 


\subsection{Results}

\subsubsection{Functionalization of PCL with VEGF-binding peptides}

An extra glycine and lysine were added at the N-termini of the VEGF-binding sequence SWWAPFH (Figure S5.1). The function of the two extra amino acids was previously reported in chapter 3. Water contact angle measurements were used in order to keep track of the chemical modifications that occurred at the surface of the PCL films (Figure 5.1). Treatment of the native PCL films with oxygen plasma led to a decrease in the water contact angle of $32 \pm 2^{\circ}(p<0.0001)$ due to the introduction of oxygen-containing groups on the surface. An increase of $18 \pm 2^{\circ}(p<0.0001)$ was observed after incubating the oxygen plasma activated films with NHS/EDC. Incubation of the amine reactive esters with $1 \mathrm{mM}$ of the VEGF-binding peptide in PBS resulted in an increase of the water contact angle of $7 \pm 2^{\circ}(p<0.01)$. In contrast, incubation with only PBS did not lead to a significant change in the water contact angle of the films with the amine reactive esters.
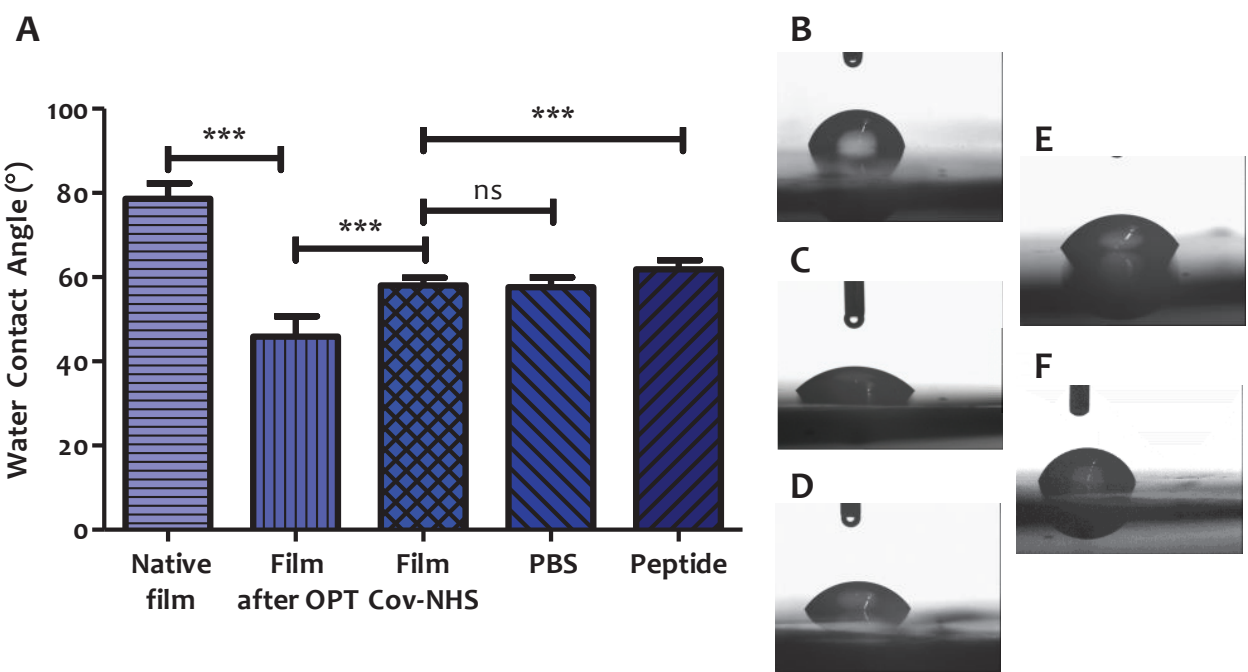

Figure 5.1. (A) Water contact angle measurements with respective representative pictures of the films during the chemical functionalization procedure; (B) 'Native film' represents the PCL films without any chemical modification; (C) 'Films after OPT' are the native PCL films exposed for 5 min to oxygen plasma; (D) 'Films Cov-NHS' indicates the films with amine reactive esters after an incubation of 1 hour with NHS/EDC; (E) 'PBS' is the films with reactive amine esters incubated for 4 hours with PBS; (F) 'Peptide' indicates the films with reactive amine esters incubated for 4 hours with a $1 \mathrm{mM}$ peptide in PBS. $* * * p<0.0001$ (two-tailed unpaired t-test). The data represent the mean \pm SD of two measurements per sample $(n=6)$. 


\subsubsection{Immobilization of VEGF onto PCL films}

An immunostaining against hVEGF was performed in order to confirm the binding of hVEGF to the VEGF-binding peptide covalently immobilized at the PCL surface. A maximum fluorescence intensity was observed in the functionalized films incubated with hVEGF (Figure 5.2A and B). This value was seven-fold higher than the non-functionalized films incubated with hVEGF (Figure 5.2A and C). Our results show that the reported fluorescence signal is solely due to the immobilization of VEGF at the surface and not due to the nonspecific interaction of the antibodies with the films. Incubation of the native film with hVEGF also led to an increase in the fluorescence signal when compared with the native films that were not incubated with hVEGF. This can be explained by nonspecific adsorption of hVEGF to the native film. In conclusion, we showed that the functionalization of PCL films with a VEGF-binding peptide allows the immobilization of this GF on the film.

A

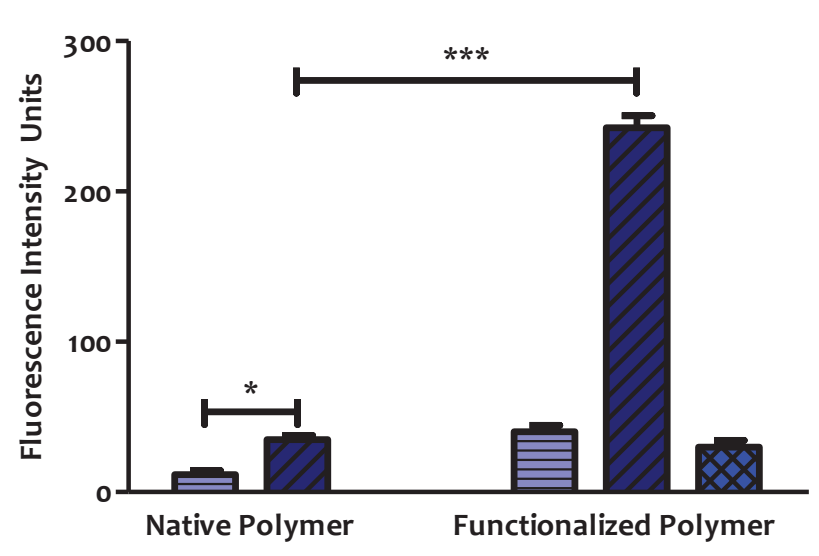

Without VEGF

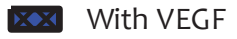

(only Secondary Ab)
B

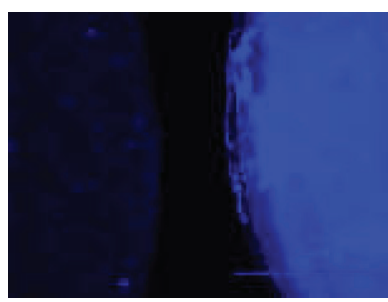

C

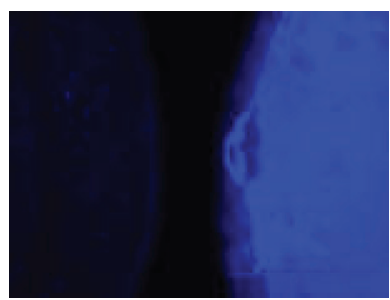

Figure 5.2. (A) Fluorescence quantification of the immunochemistry assay against hVEGF immobilized on the films. The data represent mean $\pm S D$ of three measurements per sample $(n=2)$. *** $p<0.0001$ (one-way ANOVA) with functionalized films incubated with hVEGF and immunochemistry performed with primary and secondary antibody. (B) Fluorescence image of the functionalized film without (left) vs with (right) hVEGF incubation. (C) Fluorescence image of the native film (left) vs functionalized film (right) with hVEGF incubation. Scale bar: $1000 \mu \mathrm{m}$. 


\subsubsection{Immobilized hVEGF enhances survival of HUVECS}

VEGF is known to induce the proliferation and survival of HUVECs [20]. Therefore, we determined whether the immobilized hVEGF was capable of enhancing the survival of HUVECs after a starvation period of 24 hours (Figure 5.3). HUVECs were seeded on top of native and functionalized films that were pre-incubated with o (without hVEGF) or 1 (with ihVEGF) $\mu \mathrm{g} / \mathrm{mL}$ of hVEGF and allowed to attach overnight. The following day, the medium was replaced with EBM-2 without FBS and supplemented with o or $20 \mathrm{ng} / \mathrm{mL}$ of hVEGF (ShVEGF) in the case of the films that were not pre-incubated with the GF prior to cell seeding. The medium of the remaining conditions was changed to basal medium only. After 24 hours, the total amount of DNA was quantified. As expected, treatment of the cells with soluble VEGF during the starvation period led to a significant increase in the amount of DNA when compared with the native films without hVEGF. Interestingly, our results showed that the amount of hVEGF that remained nonspecifically adsorbed to the native film did not lead to an increase in the number of cells after the starvation period. This may be explained by the fact that the surface concentration of the hVEGF is insufficient to trigger a cell response or by a loss of GF bioactivity due to conformational changes during the adsorption process. In contrast, preincubation of the functionalized films with hVEGF enhanced ECs survival, as measured by the total amount of DNA. This result shows that the VEGF captured by the VEGF-binding peptide remained bioactive and induced the survival of HUVECs during the starvation period. There was no statistical significance observed between the results from the functionalized films with immobilized VEGF and the native/functionalized films with soluble VEGF. In summary, the hVEGF captured by the VEGF-binding peptide retains its bioactivity by inducing a cell response in HUVECs. 


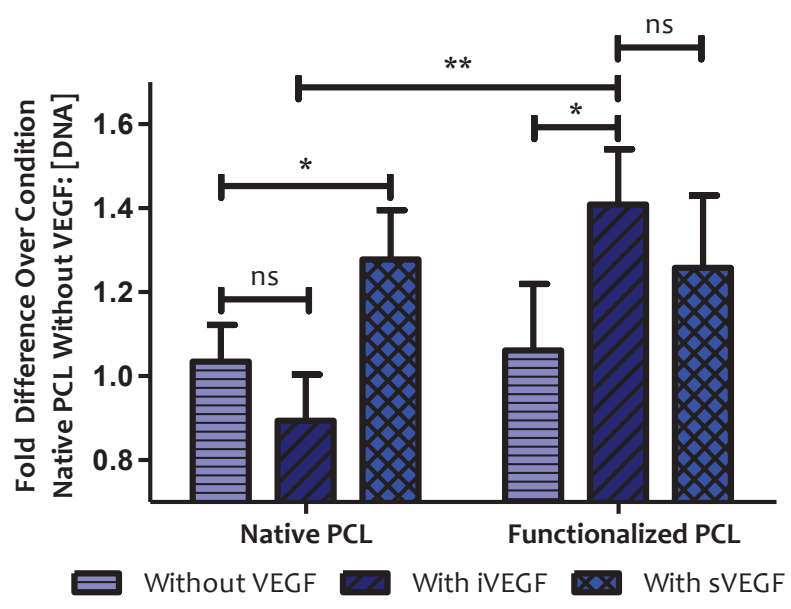

Figure 5.3. The effect of the immobilized (ihVEGF) and soluble (shVEGF) VEGF in the survival of HUVECS cultured in native and functionalized films. ${ }^{*} p<0.05, * * p<0.01$ (two-tailed unpaired t-test). The data represent the mean $\pm S D$ of two measurements per sample $(n=3)$.

\subsubsection{In vivo capture of endogenous VEGF by the VEGF-binding peptide leads to the appearance of blood vessel-like structures}

Several cytokines, such as interleukin- 6 and -8 (IL-6 and -8 ) and tumour necrosis factor alpha (TNF- $\alpha$ ), which are produced during the inflammatory stage, are known to upregulate VEGF expression [21]. We hypothesized that the VEGF-binding peptide could capture and accumulate the VEGF that naturally occurs in the body or/and that is produced in response to inflammatory cytokines, leading to a more pronounced tissue response. Both native and functionalized PCL films were subcutaneously implanted in the backs of rats, facing the fascia. Each animal, with a total of three animals per time point, was implanted with both native and functionalized film. At day 3 and day 7 after implantation, samples were harvested and used for immunofluorescence against VEGF and histology. Only samples from day 7 were used for immunofluorescence against VEGF. A higher intensity signal was found in the functionalized films at day 7 post implantation in all three animals (Figure 5.4), showing that an increased amount of VEGF was found around the functionalized implant when compared with the native implant. 
A

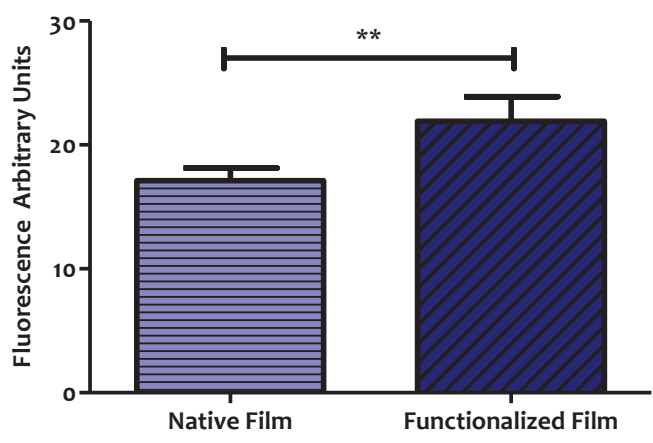

B

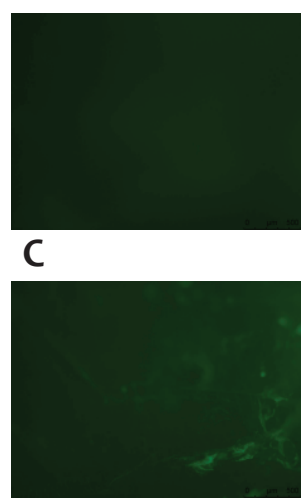

Figure 5.4. (A) Fluorescence quantification of the immunochemistry assay against VEGF immobilized on implanted films. The data represent mean \pm SD of five measurements per sample $(n=3) .{ }^{* *} p<0.01$ (two-tailed unpaired t-test). (B) Fluorescence image of the native film implanted subcutaneously in rats. (C) Fluorescence image of the film functionalized with VEGF-binding peptide implanted subcutaneously in rats.

From our histological results, the accumulation of VEGF in the functionalized films resulted in a higher cellularity - the majority being inflammatory cells - and the appearance of blood vessel-like structures (yellow arrows) at day 3, when compared with the native films (Figure 5.5 A-D). After 7 days, the tissue around the functionalized films showed it to be structurally more organized, with a high degree of vascularization (Figure 5.5 E-F). Overall, these results show that the accumulation of VEGF by the peptide led to the more pronounced recruitment of inflammatory cells and the appearance of blood vessel-like structures after 3 days, eventually leading to a more organized tissue around the functionalized implant after 7 days. 


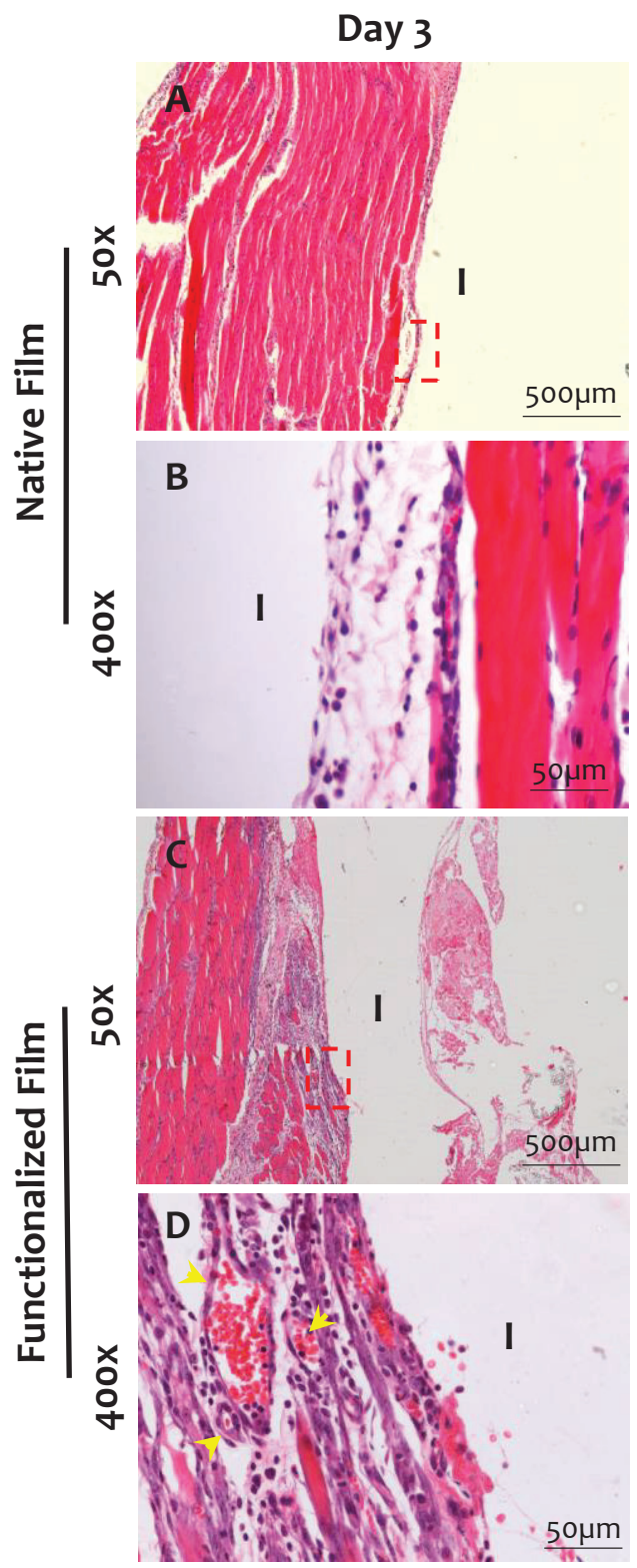

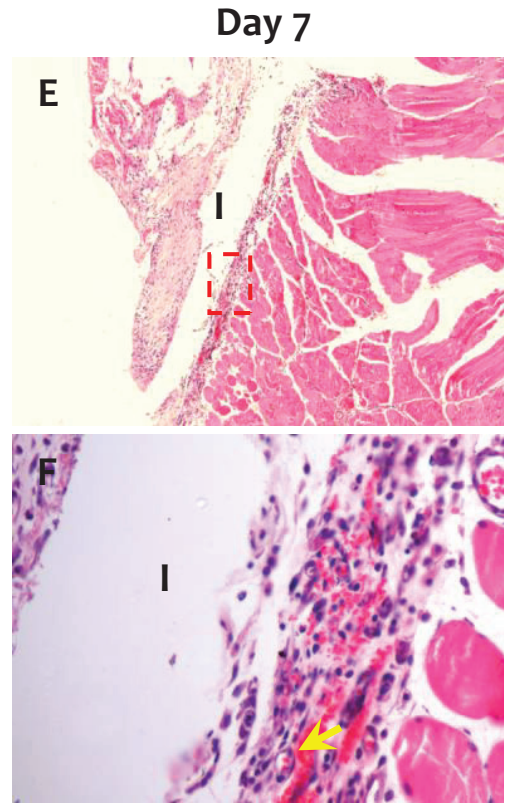
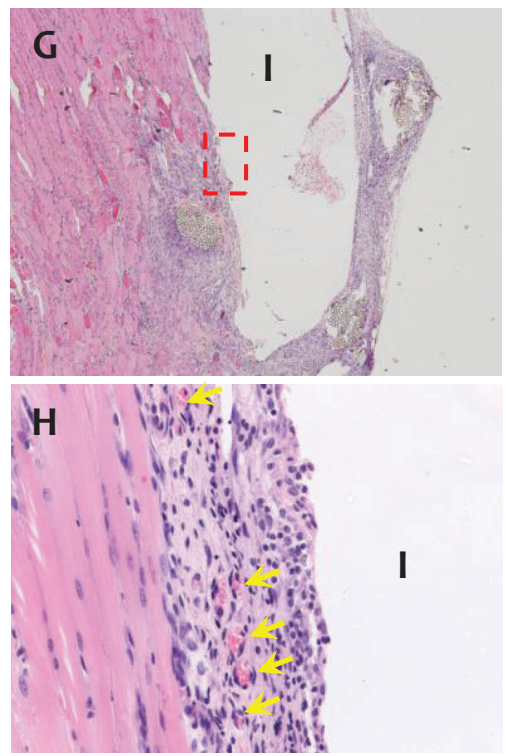

Figure 5.5. Histological evaluation of sample sections $3(A-D)$ and $7(E-H)$ days after implantation. Haematoxylin and eosin staining on native films ( $A, B, E$ and $F)$ and functionalized films (C, G, D and H). Implant (I), blood vessels (yellow arrows), magnified area (red dashed box). 


\subsubsection{Functionalization of orthopaedic medical devices with VEGF-binding peptides allows the immobilization of VEGF}

Tissue healing requires cell infiltration from the blood stream to the tissue in order to induce the healing process. Vascularized tissues have increased healing abilities, due to higher exchange rates of nutrients and metabolic waste, and cell infiltration and migration. Tissues such as tendons, ligaments and meniscus are poorly vascularized and consequently have very low healing capacities. Therefore, we selected several medical devices that are currently used in orthopaedic surgeries and modified them in order to covalently immobilize the VEGF-binding peptide (Figure 5.6). Next, we incubated the devices with hVEGF (Figure 5.7) and quantified the fluorescence signal from the immunostaining against the GF (Figure 5.8). The medical devices used were Poly-tape from iMove Medical $^{\mathrm{TM}}$, which is a polyester mesh designed for soft tissue ingrowth and neoligament formation, Chondro-Gide from Geistlich Pharma $\mathrm{AG}^{\mathrm{TM}}$, a collagen scaffold intended to be used for the treatment of articular cartilage defects, and Collagen Meniscus Implant ( $\mathrm{CMI}$ ) from IvySports Medicine ${ }^{\mathrm{TM}}$, which is intended to fill the void resulting from damage to and/or loss of meniscal tissue.
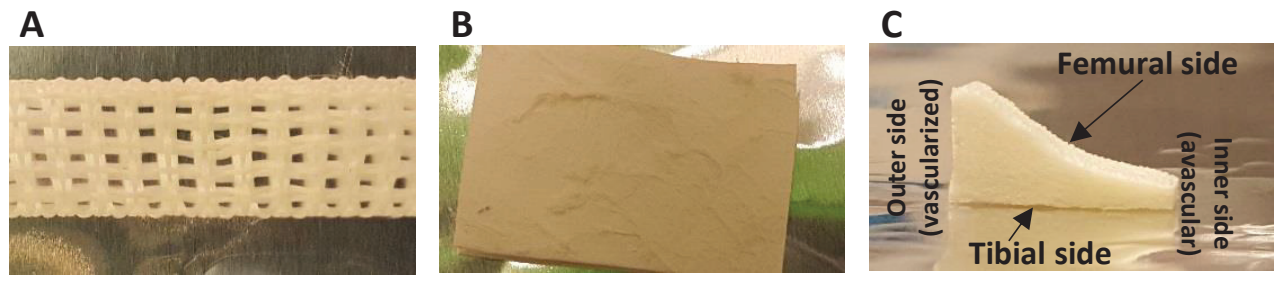

Figure 5.6. Picture of the (A) Poly-tape material, (B) Chondro-Gide and (C) CMI.

The Poly-tape was oxygen plasma treated (OPT) for $5 \mathrm{~min}$ and incubated for one hour with $1 \mathrm{M} \mathrm{NaOH}$ in order to hydrolyse the esters and expose the carboxylic acids. Next, the Poly-tape was incubated with NHS/EDC for one hour and finally with $1 \mathrm{mM}$ of the VEGF-binding peptide in PBS for 2 hours. Incubation of the native Poly-tape with hVEGF led to a significant increase in the fluorescence signal when compared with the same films without hVEGF incubation, showing that this polyester is prone to protein adsorption. Treatment of the Poly-tape with oxygen plasma increased the surface hydrophilicity and consequently led to further unspecific adsorption of the hVEGF when compared with the native Poly-tape that was also incubated with hVEGF $(p<0.01)$. However, the fluorescence signal was maximal when the Poly-tape functionalized with the VEGF-binding peptide was incubated with hVEGF, which was also 2.3 times higher 
than the native Poly-tape with hVEGF $(p<0.001)$ and 1.5 times higher than the Poly-tape with OTP incubated with hVEGF $(p<0.001)$. In the case of the Chondro-Gide, the scaffold was incubated with NHS/EDC for 2 hours in order to react with the carboxylic acids of the collagen proteins and subsequently incubated with the VEGF-binding peptide in PBS for a further 2 hours. Incubation of the native Chondro-Gide with hVEGF did not lead to an increase in the fluorescence signal, showing that this scaffold is unsuitable to function as a VEGF delivery system. However, modification of the carboxylic acids of the collagen proteins with NHS/EDC in order to introduce amine reactive esters allowed the immobilization of the hVEGF on the scaffold. This increase in the fluorescence signal may be due to the reaction of the free amines of the hVEGF with the amine reactive esters and/or due to unspecific adsorption of the hVEGF to the scaffold. Notwithstanding, it was when the material was functionalized with the VEGF-binding peptide that the highest fluorescence signal was observed, which was also 2.2 times higher than the value observed for the Chondro-Gide functionalized with NHS/EDC and incubated with hVEGF $(p<0.001)$. The CMI, similarly to the Chondro-Gide, is a collagen-based material and therefore the chemical strategy of immobilizing the VEGF-binding peptide on it was the same used for the Chondro-Gide. In contrast to the Chondro-Gide, incubation of the native CMI with hVEGF led to an increase in the fluorescence signal due to the unspecific adsorption of the GF to the scaffold. However, the fluorescence signal was 3.3 times higher when the CMI functionalized with the VEGF-binding peptide was incubated with hVEGF. Taken together, these results show that medical devices that are currently used in the clinic can be redesigned and improved with the addition of a VEGF-binding peptide, in order to deliver and/or capture hVEGF and consequently to induce angiogenesis and the healing of tissues such as $\mathrm{T} / \mathrm{L}$, cartilage and meniscus that have very low healing capacities due to their inherently poor vascular networks. 


\section{Chapter 5 Improvement of medical devices with VEGF-binding peptides in order to induce vascularization in orthopaedic injuries}

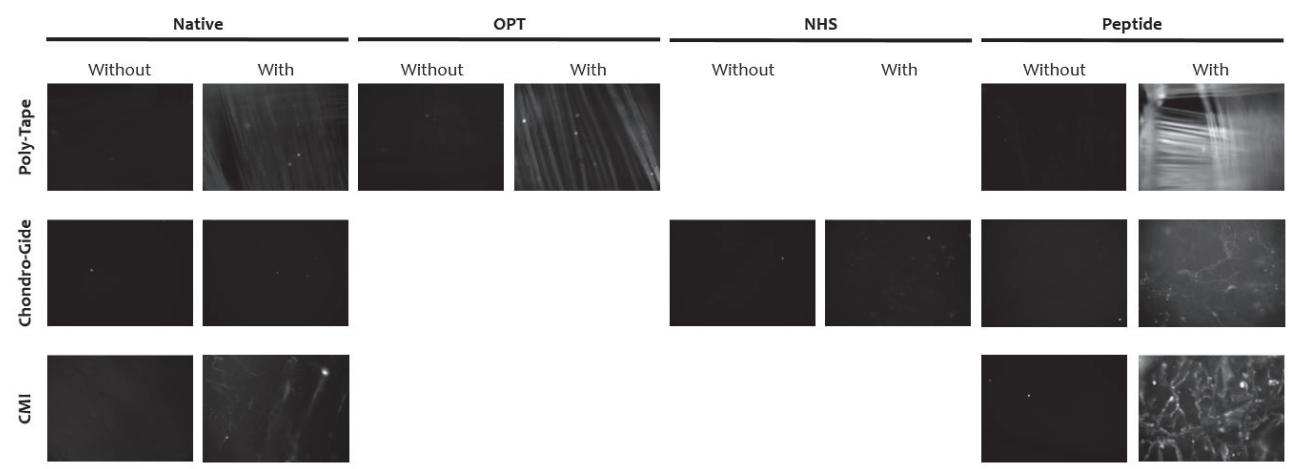

Figure 5.7. Immunofluorescence pictures of Poly-Tape, Chondro-Gide and CMI that were incubated with o (without) or $1 \mu \mathrm{g} / \mathrm{mL}$ (with) of hVEGF. 'Native' refers to the device without any chemical modification, 'OTP' is the device after oxygen plasma treatment, 'NHS' indicates the device with amine reactive esters and 'Peptide' refers to the device with the VEGF-binding peptide covalently immobilized on it.

A

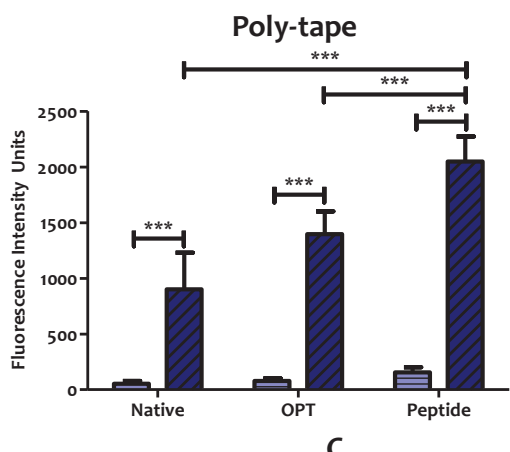

C

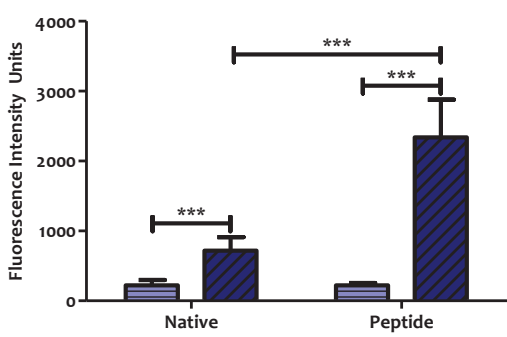

Without VEGF
B

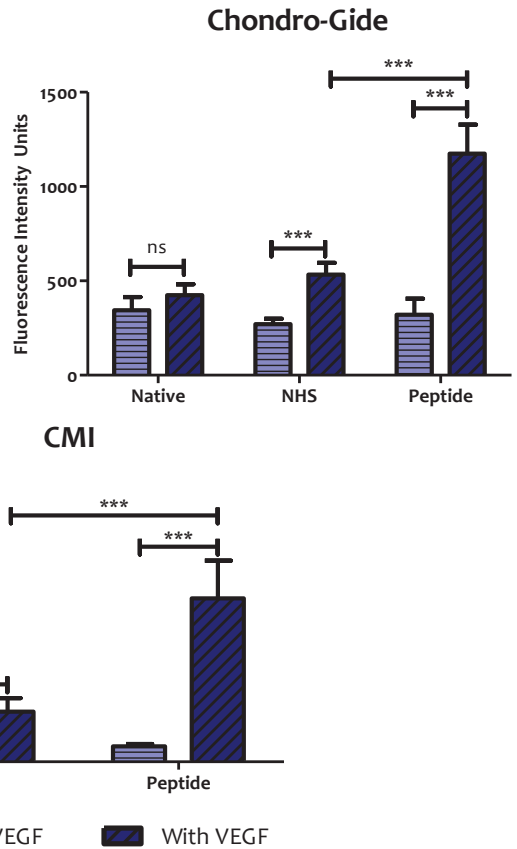

Figure 5.8. Fluorescence quantification of the immunostaining against hVEGF on (A) Poly-Tape, (B) Chondro-Gide and (C) CMI that were incubated with o (without) and $1 \mu \mathrm{g} / \mathrm{mL}$ (with) of hVEGF. *** $p<0.001$ (two-tailed unpaired t-test). The data represent the mean \pm SD per sample $(n=5)$. 


\subsubsection{Engineering hVEGF immobilization in functionalized CMIs in order to mimic the meniscus's vascular network distribution}

In the adult meniscus, vascular penetration is only $10-30 \%$ of the width of the medial meniscus and $10-25 \%$ of the width of the lateral meniscus. Two distinct zones can be identified in terms of vascularization: an outer, vascularized zone and an inner, avascular zone [22]. Therefore, spatial presentation of hVEGF in functionalized CMIs is required to mimic the vascular network of the meniscus. The outer side of the functionalized CMIs was placed on top of a layer of solution containing $1 \mu \mathrm{g} / \mathrm{mL}$ of hVEGF and contact was allowed to occur for 10 and $60 \mathrm{~min}$. The scaffold acted as a sponge, and due to capillary forces the hVEGF solution moved upwards in the direction of the inner side. A gradient of the GF can therefore be achieved depending on the time that the CMI is allowed to contact the hVEGF solution. Next, the scaffolds were stained against hVEGF and the outer (Figure 5.9A), femoral (Figure 5.9B) and tibial side (Figure. 5.9C) of the scaffolds were imaged. In the cases of the tibial and femoral sides, the images were taken from the inner to the outer side of the scaffold. The lateral outer side of the CMI, which was in direct contact with the layer of hVEGF solution, had a homogeneous distribution of the GF from the tibial to the femoral side. When the CMI was in contact with the hVEGF solution for $10 \mathrm{~min}$, there was a higher concentration of hVEGF in the core of that side, while the amount was lower in the border areas. However, when the CMI was allowed to be in contact with the hVEGF for a longer period, there was no difference in fluorescence between the core and the border areas. In both cases, the slope deviation from zero was not statistically significant. Regarding the femoral and the tibial sides of the CMI, an hVEGF gradient was achieved from the inner to the outer side when the CMI was in contact with the hVEGF solution for $10 \mathrm{~min}$. The inner side of the CMI, which corresponded to the avascular side of the meniscus, and the side that was furthest away from the hVEGF solution, showed a lower fluorescence intensity. The fluorescence increased in the direction towards the outer side, which corresponded to the vascularized side of the meniscus, and which was in direct contact with the hVEGF solution. Both the femoral $(p<0.0001)$ and the tibial $(p=0.0003)$ side of the $C M I$ that were in contact for 10 min with the hVEGF solution showed results that were statistically significantly different from zero. When the CMI was allowed to be in contact with the hVEGF solution for a longer period, the concentration gradient vanished. In the case of the femoral side, the hVEGF became homogenously distributed from the inner to the outer side, with a slope not statistically significantly different from zero. In contrast, the tibial side had a higher amount of hVEGF at the inner side. In summary, these results 
show that the distribution of hVEGF in CMI devices that were functionalized with a VEGFbinding peptide was able to be controlled in order to achieve gradients of hVEGF and consequently to supply different areas of the meniscus with varying amounts of GF according to the natural vascular network distribution within this tissue.

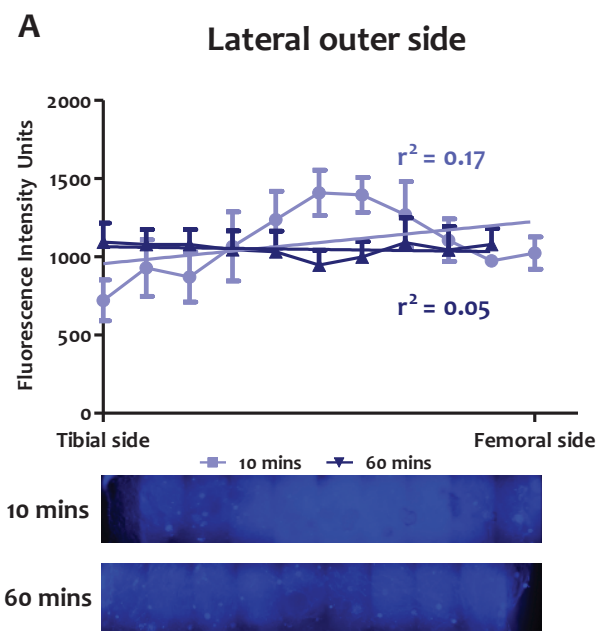

B Tibial Side
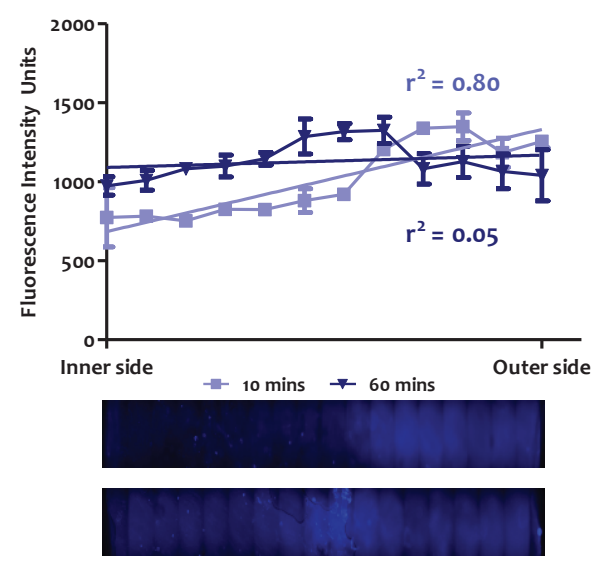

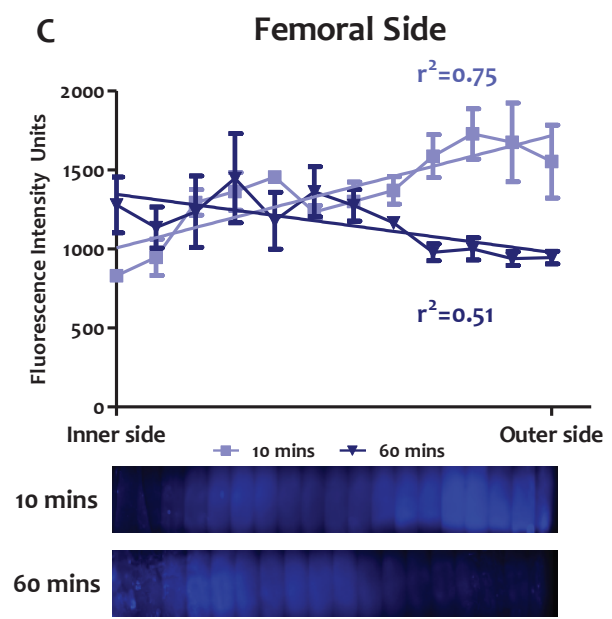

Figure 5.9. Fluorescence quantification and imaging of the immunostaining against $h V E G F$ of the $(A)$ lateral outer side, (B) the femoral side, and (C) the tibial side of functionalized CMIs that were in contact with a solution of hVEGF for $10 \mathrm{~min}$ (grey line) and $60 \mathrm{~min}$ (dark line). Values are represented as mean $\pm S D(n=3)$. 


\subsection{Discussion}

Here we hypothesized that the functionalization of biomaterials with a VEGF-binding peptide would allow the capture and presentation of hVEGF to cells and tissues. We have here shown that it is possible to non-covalently immobilize hVEGF onto PCL films that had been functionalized with a VEGF-binding peptide. The immobilized hVEGF remained bioactive and was capable of increasing the survival of ECs seeded onto the films in vitro. When the functionalized films were implanted in rats, the VEGF-binding peptide was able to capture endogenous VEGF, eliciting an increase in the number of blood vessels around the implant. Ultimately, we have shown that the medical devices that are currently used for injuries to T/L, cartilage and meniscus can be functionalized with the VEGF-binding peptide. We envision that these functionalized medical devices will capture the patient's VEGF and, by enhancing the formation of blood vessels, overcome the poor healing capacity associated with poorly vascularized tissues.

Injuries to tissues such as T/L, meniscus and cartilage can be extremely painful and debilitating for the patient [23-25]. Additionally, the poor healing capacity of these tissues slows or compromises their overall healing process when compared with other tissues in the body. Connective tissue has a very poor healing capacity, which is why $\mathrm{T} / \mathrm{L}$ heal slowly [26]. Articular cartilage also has a low healing and repair ability due to its lack of blood vessels, lymphatics and nerves, and due to the harsh biomechanical environment to which it is subjected [27]. The blood supply is also critical for the healing and repair ability of the meniscus [28]. The meniscus receives its vascular supply from the medial, lateral and middle geniculate arteries; however, these arteries only cover 10 to $30 \%$ of the peripheral medial meniscus border and $10 \%$ to $25 \%$ of the lateral meniscus border [28]. The remaining meniscus is avascular and consequently cannot self-heal [22]. It is well established that the healing capacity of these tissues is ultimately dependent on their vascular network. Therefore, several studies exploiting various strategies have been conducted to study and promote the angiogenic process in these tissues, in order to ultimately enhance their healing and repair. One strategy often employed to induce blood vessel formation is the use of pro-angiogenic factors such as VEGF. VEGF is produced and released by many cells types that participate in wound healing, such as endothelial cells, fibroblasts, platelets and macrophages [29]. Within the healing cascade, VEGF is involved in vasodilation, basement membrane degradation, cell migration and proliferation, and capillary tube formation and sprouting [29]. One study showed that VEGF is expressed in autologous tendon grafts after anterior cruciate ligament $(\mathrm{ACL})$ reconstruction, and that it is a key player in the remodelling of tendon 
grafts [30]. However, strategies relying on the soluble delivery of VEGF should be avoided. As the half-life of VEGF within the body is only $30 \mathrm{~min}$, supraphysiological amounts and multiple injections are needed [31]. The use of such high amounts, combined with diffusion inside the body, can potentially lead to pathological vessel formation in non-target tissues. One way to avoid these issues is by immobilizing or encapsulating GFs onto biomaterials. For example, Golub et al. (2010) showed that the sustained release of VEGF from poly(lactic-co-glycolic acid) (PLGA) nanoparticles promoted blood vessel growth in a mouse femoral artery ischemia model [32]. In the current study, we described a system to non-covalently deliver hVEGF via the interaction with a synthetic VEGF-binding peptide. Various biomaterials were chemically functionalized in order to covalently immobilize the VEGF-binding peptide, the amount of captured hVEGF was quantified by immunofluorescence and both the in vitro and in vivo biological responses were assessed.

First, we synthetized the VEGF-binding peptide with the sequence KGSWWAPFH and acetylated the $\mathrm{N}$-termini, which left the side chain of the lysine amino acid as a reactive group for the covalent immobilization strategy. PCL films were used for in vitro experiments. Each chemical modification at the surface of the films was confirmed with water contact angle measurements. The affinity of the VEGF-binding peptide towards VEGF was confirmed by immunochemistry. From our results, we can conclude that some of the hVEGF added to the native film remained unspecifically adsorbed due to the increase in the fluorescence intensity. However, incubation of the PCL functionalized with a VEGF-binding peptide with hVEGF led to an increase in the fluorescence intensity that was seven times higher than the fluorescence reported for the native films incubated with hVEGF. These results show that is possible to non-covalently immobilize hVEGF on biomaterials via interaction with the VEGF-binding peptide. One drawback to the immobilization of GFs onto biomaterials is their loss of bioactivity. VEGF is known to act as a pro-survival and mitogenic cytokine in HUVECs [33]. Therefore, we next cultured HUVECs on top of the films, starved them for 24 hours after adherence and then quantified the amount of DNA. Our results showed that there was no difference between native films and functionalized films without hVEGF incubation in terms of the number of cells after starvation. Differences in hydrophobicity could affect cell adhesion and consequently the number of cells after starvation. The highest amount of DNA was found in the functionalized films with immobilized hVEGF. This value was 1.5 times higher than the amount of DNA found in the native films incubated with hVEGF before cell seeding $(p<0.01)$. The amount of DNA in the conditions that were treated with soluble hVEGF was not statistically significantly different from the value of DNA reported for the 
functionalized films with immobilized hVEGF. These data show that the hVEGF captured and immobilized by the VEGF-binding peptide promoted the survivability of HUVECS after a period of starvation.

In this study, we hypothesized that functionalizing biomaterials with a VEGF-binding peptide would allow the implanted biomaterial to capture endogenous VEGF and accumulate it, leading to a response from the surrounding tissue. This could allow the avoidance of the administration of exogenous GFs, therefore reducing the risks associated with those strategies. VEGF has a key role in wound healing immediately after injury through promoting early angiogenic events, such as vascular dilation, permeability, migration and proliferation, during a time window of 3 to 7 days [18]. To test this hypothesis, we subcutaneously implanted films of native and functionalized PCL in the back of rats. After 3 and 7 days the implants were harvested and histologically stained. The implants harvested 7 days after implantation were also immunostained against VEGF. Our immunochemistry results show that the $P C L$ films functionalized with the VEGF-binding peptide had a higher fluorescence signal, showing that the peptide captured and accumulated the VEGF onto the film. This VEGF accumulation was eventually translated into a more pronounced healing response by the surrounding tissue. In all of the animals, there was an increase in cells (mostly inflammatory cells) around the functionalized films at day 3. An increase in blood vessel-like structures around the functionalized films was also observed. After 7 days, an increased amount of cells was again found around the functionalized films. Overall, the surrounding tissue of the functionalized film was more structurally organized, with a denser blood vessel network than the native implants. VEGF is known to act as a chemoattractant and to induce the migration of cells. Zhang et al. (2014) showed that silk scaffold containing VEGF induced the homing of bone marrow stem cells (BMSCs) that had been injected into the veins of the mouse tail to the scaffold location [34]. VEGF also stimulates the migration of endothelial cells [35]. The endogenous VEGF captured by the VEGF-binding peptide could have attracted inflammatory and endothelial cells to the injury site, leading to the higher cellularity and to the appearance of the blood vessel-like structures observed after 3 days. This early attraction and migration of cells, combined with a more pronounced capillary network, resulted in the higher organization of the tissue around the functionalized implant after 7 days. These results show that the functionalization of biomaterials with a VEGF-binding peptide gives them the capacity to capture endogenous VEGF and consequently to enhance its local concentration. 
Next, we functionalized three different medical devices with the VEGF-binding peptide to assess whether it was possible to immobilize hVEGF on them. The selected devices were: Poly-tape, Chondro-Gide and CMI. Poly-tape is used to repair ruptured tendons, Chondro-Gide for the treatment of articular cartilage, and the CMI is used to replace damaged meniscus or to fill the void left by the partial removal of meniscus. For each of these three materials, the highest value of fluorescence intensity was reported when the medical device was functionalized with the VEGF-binding peptide and was incubated with hVEGF. These data clearly show that current medical devices can be redesigned and/or improved by functionalizing them with GF-binding peptides, allowing them to capture and deliver the GF to cells and tissues. One previous study has shown that using VEGF gene therapy via adeno-associated viral type 2 vector to produce high amounts of VEGF in transversely-lacerated digital flexor tendons of chickens led to an increase in the production of collagen type I, cellular proliferation and tendon strength by $82-210 \%$ after 3 weeks when compared with controls [36]. Despite the fact that VEGF is crucial to neonatal endochondral bone development, its expression becomes quiescent in adult human articular cartilage [37]. In fact, VEGF expression in adult cartilage is associated with cartilage-related pathology. Lingaraj et al. (2010) showed that VEGF is re-expressed in osteoarthritis [38]. One study showed that the neutralization of VEGF using humanized monoclonal anti-VEGF antibody contributed to the improved healing of articular cartilage in a rabbit osteochondral defect model [39]. Alternatively, the VEGFbinding peptide could be used in the treatment of various diseases by working as a filtration system, removing VEGF. Materials containing the VEGF-binding peptide could be used to remove target VEGF from the patient's joint or from the synovial fluid. In the case of the CMI, it is undesirable to have a homogenous distribution of hVEGF along the scaffold due to the distribution of the vascular network in the meniscus. We have here shown that, through capillary forces, it is possible to create gradients of hVEGF in CMIs functionalized with the VEGF-binding peptide. This strategy allowed the creation of a zone of high amounts of immobilized hVEGF in the outer side of the CMI that matched the outer and vascularized area of the meniscus, and a low concentration area in the inner side of the CMI that corresponded to the inner, avascular area of the meniscus, with a gradient of immobilized hVEGF between these two distinct areas. The gradient slope was able to be tuned by controlling parameters such as hVEGF concentration or the time for which the CMI was allowed to be in contact with the hVEGF solution. Here, we demonstrated a strategy to immobilize and deliver hVEGF via the affinity towards a VEGF-binding peptide. Aspects such as the amount of hVEGF immobilized in functionalized materials can be controlled by tuning the number of available epitopes of the peptide. Functionalization of biomaterials with the VEGF-binding peptide could 
potentially allow the material to capture endogenous VEGF, accumulate it onto the material and consequently correct the insufficient healing capacity of the ruptured tendon.

\subsection{Conclusions}

In this work, we investigated the immobilization and delivery of VEGF in various biomaterials via interaction with a VEGF-binding peptide. In vitro studies showed that the bioactivity of the immobilized VEGF was not lost and that it induced the survivability of cells. In vivo studies showed that implants functionalized with the VEGF-binding peptide captured the endogenous GF, leading to its accumulation around the implant. This accumulation of VEGF resulted in the higher recruitment of inflammatory cells and in neovascularization at day 3 , which ultimately led to a higher organization of the tissue around the functionalized implant at day 7 . We also showed that medical devices that are currently used in standard orthopaedic surgeries can be modified with the VEGFbinding peptide, turning them into a VEGF capturing device. Once implanted in the patient, the functionalized medical devices would capture endogenous VEGF and consequently trigger vascularization and enhance the healing of avascular tissues.

\subsection{Acknowledgments}

We acknowledge Smith\&Nephew for providing financial support for these investigations. We would also like to thank Dr. Bruma Fu and Angel Lee from the University of Hong Kong for their collaboration in the in vivo experiments. Albert Jan Stegeman is also acknowledged for his contribution to the laboratory work. 


\subsection{References}

1. Lovett $M$, et al., Vascularization strategies for tissue engineering. Tissue Eng Part B Rev, 2009. 15(3): p. 353-70.

2. Novosel ES, Kleinhans C, and Kluger PJ, Vascularization is the key challenge in tissue engineering. Adv Drug Deliv Rev, 2011. 63(4-5): p. 300-11.

3. Rouwkema J, Rivron NC, and van Blitterswijk CA, Vascularization in tissue engineering. Trends Biotechnol, 2008. 26(8): p. 434-41.

4. Baranski JD, et al., Geometric control of vascular networks to enhance engineered tissue integration and function. PNAS, 2013. 110(19): p. 7586-91.

5. Sharma P and Maffulli N, Biology of tendon injury: healing, modeling and remodeling. J Musculoskelet Neuronal Interact, 2006. 6(2): p. 181-90.

6. Camp CL, Stuart MJ, and Krych AJ, Current Concepts of Articular Cartilage Restoration Techniques in the Knee. Sports Health, 2014. 6(3): p. 265-273.

7. Rouwkema J and Khademhosseini A, Vascularization and Angiogenesis in Tissue Engineering: Beyond Creating Static Networks. Trends Biotechnol, 2016(16).

8. Hoeben A, et al., Vascular endothelial growth factor and angiogenesis. Pharmacol Rev, 2004. 56(4): p. 549-80.

9. Raiter A, et al., Angiogenic peptides improve blood flow and promote capillary growth in a diabetic and ischaemic mouse model. Eur J Vasc Endovasc Surg, 2010. 40(3): p. 381-8.

10. Mammadov R, et al., Heparin mimetic peptide nanofibers promote angiogenesis. Biomacromolecules, 2011. 12(10): p. 3508-19.

11. Griffith LG and Naughton G, Tissue engineering--current challenges and expanding opportunities. Science, 2002. 295(5557): p. 1009-14.

12. Olsson AK, et al., VEGF receptor signalling - in control of vascular function. Nat Rev Mol Cell Biol, 2006. 7(5): p. 359-71.

13. M, S., Vascular Endothelial Growth Factor (VEGF) and Its Receptor (VEGFR) Signaling in Angiogenesis: A Crucial Target for Anti- and Pro-Angiogenic Therapies. Genes Cancer, 2011. 2(12): p. 1097-105.

14. Ferrara N, Role of vascular endothelial growth factor in regulation of physiological angiogenesis. Am J Physiol Cell Physiol, 2001. 280(6): p. C1358-66.

15. Shen $\mathrm{YH}$, Shoichet MS, and Radisic M, Vascular endothelial growth factor immobilized in collagen scaffold promotes penetration and proliferation of endothelial cells. Acta Biomater, 2008. 4(3): p. 477-89.

16. Miyagi $\mathrm{Y}$, et al., Biodegradable collagen patch with covalently immobilized VEGF for myocardial repair. Biomaterials, 2011. 32(5): p. 1280-90.

17. Hu X, et al., Immobilization strategy for optimizing VEGF's concurrent bioactivity towards endothelial cells and osteoblasts on implant surfaces. Biomaterials, 2012. 33(32): p. 808293.

18. Webber MJ, et al., Supramolecular nanostructures that mimic VEGF as a strategy for ischemic tissue repair. PNAS, 2010. 108(33): p. 13438-13443.

19. Stupp SI, et al., Self-assembling peptide amphiphiles and related methods for growth factor delivery N. University, Editor 2009.

20. Tammela T, et al., The biology of vascular endothelial growth factors. Cardiovasc Res, 2005. 65(3): p. 550-63. 
21. Maloney JP and Gao L, Proinflammatory Cytokines Increase Vascular Endothelial Growth Factor Expression in Alveolar Epithelial Cells. Mediators Inflamm, 2015. 2015.

22. Makris EA, Hadidi P, and Athanasiou KA, The knee meniscus: structure-function, pathophysiology, current repair techniques, and prospects for regeneration. Biomaterials, 2011. 32(30): p. 7411-31.

23. Bass E, Tendinopathy: Why the Difference Between Tendinitis and Tendinosis Matters. Int J Ther Massage Bodywork, 2012. 5(1): p. 14-17.

24. Logerstedt DS, et al., Knee pain and mobility impairments: meniscal and articular cartilage lesions. J Orthop Sports Phys Ther, 2010. 40(6): p. A1-A35.

25. Bhatia D, Bejarano T, and Novo M, Current interventions in the management of knee osteoarthritis. J Pharm Bioallied Sci, 2013. 5(1): p. 30-8.

26. Yang G, Rothrauff BB, and Tuan RS, Tendon and ligament regeneration and repair: clinical relevance and developmental paradigm. Birth Defects Res C Embryo Today, 2013. 99(3): p. 203-22.

27. Fox AJS, Bedi A, and Rodeo SA, The Basic Science of Articular Cartilage. Sports Health, 2009. 1(6): p. 461-468.

28. Brindle T, Nyland J, and Johnson DL, The Meniscus: Review of Basic Principles With Application to Surgery and Rehabilitation. J Athl Train, 2001. 36(2): p. 160-169.

29. Bao P, et al., The Meniscus: Review of Basic Principles With Application to Surgery and Rehabilitation. J Surg Res, 2009. 153(2): p. 347-58.

30. Petersen W, et al., The angiogenic peptide vascular endothelial growth factor (VEGF) is expressed during the remodeling of free tendon grafts in sheep. Arch Orthop Trauma Surg, 2003. 123(4): p. 168-74.

31. Lee K, Silva EA, and Mooney DJ, Growth factor delivery-based tissue engineering: general approaches and a review of recent developments. J R Soc Interface, 2011. 8(55): p. 153-70.

32. Golub JS, et al., Sustained VEGF delivery via PLGA nanoparticles promotes vascular growth. Am J Physiol Heart Circ Physiol, 2010. 298(6): p. H1959-65.

33. Shu Q, et al., Vasostatin Inhibits VEGF-Induced Endothelial Cell Proliferation, Tube Formation and Induces Cell Apoptosis under Oxygen Deprivation. Int J Mol Sci, 2014. 15(4): p. 6019-30.

34. Zhang W, et al., VEGF and BMP-2 promote bone regeneration by facilitating bone marrow stem cell homing and differentiation. Eur Cell Mater, 2014. 15(27): p. 1-12.

35. Lamalice L, Boeuf FL, and Hout J, Endothelial Cell Migration During Angiogenesis. Circ Res, 2007. 100: p. 782-794.

36. Tang, J.B., et al., Basic FGF or VEGF gene therapy corrects insufficiency in the intrinsic healing capacity of tendons. Scientific Reports, 2016. 6: p. 20643.

37. Murata M, Yudoh K, and Masuko K, The potential role of vascular endothelial growth factor (VEGF) in cartilage: how the angiogenic factor could be involved in the pathogenesis of osteoarthritis? Osteoarthritis Cartilage, 2008. 16(3): p. 279-86.

38. Lingaraj K, Poh CK, and W. W, Vascular endothelial growth factor (VEGF) is expressed during articular cartilage growth and re-expressed in osteoarthritis. Ann Acad Med Singapore, 2010. 39(5): p. 399-403.

39. Nagai T, et al., Intravenous administration of anti-vascular endothelial growth factor humanized monoclonal antibody bevacizumab improves articular cartilage repair. Arthritis Res Ther, 2010. 12(5). 

Chapter 5 Improvement of medical devices with VEGF-binding peptides in order to induce vascularization in orthopaedic injuries

\subsection{Supporting information}

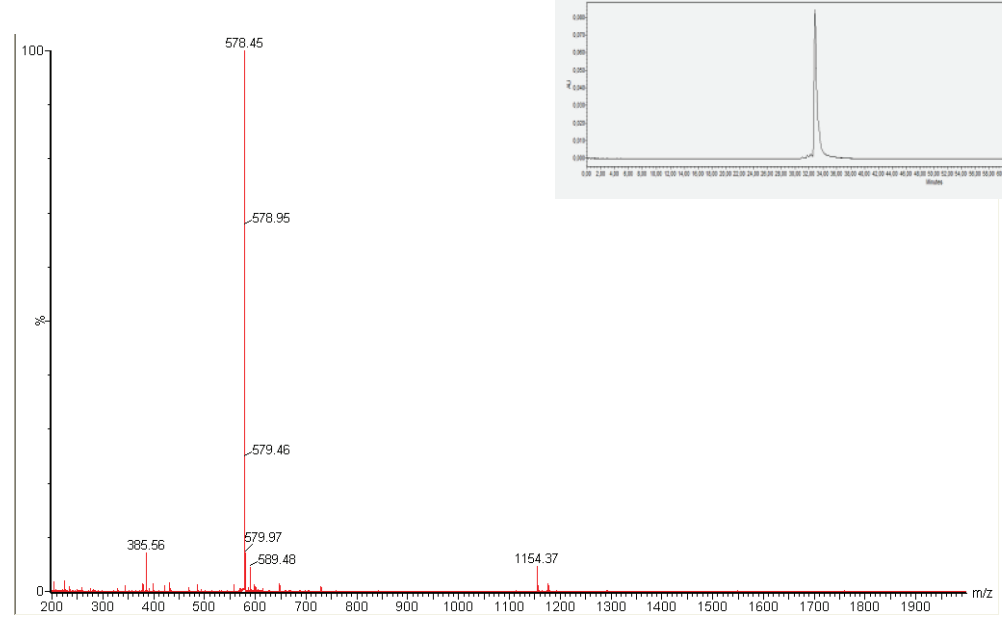

Figure S5.1. Mass spectroscopy of the VEGF-binding peptide after purification and respective HPLC. 
108 


\section{Chapter 6}

\section{Spatially presenting hTGF- $\beta 1$ on biopolymeric films}

Tissue interfaces are crucial for the proper functioning of an organ. They are composed of multiple cell types, a spatial arrangement of extracellular matrix (ECM) components and a spatial-temporal distribution of bioactive molecules that includes growth factors (GFs). Towards the development of such tissue interfaces, polycaprolactone ( $P C L$ ) films were fabricated that displayed gradients of human transforming growth factor $B_{1}$ (hTGF- $\mathrm{B}_{1}$ ). The $P C L$ films were chemically conditioned to allow a covalent reaction between the surface of the PCL films and a suitably functionalized synthesized TGF-61-binding peptide. Gradients of the TGF-B1-binding peptide were generated by lifting PCL films, during the reaction, out of solution using an automated dip coater with controlled speed. Gradient formation on the films was confirmed using X-ray photoelectron spectroscopy (XPS), and the hTGF-81 gradients were confirmed by antibody staining. The results demonstrate that a simple and easily accessible strategy was developed to generate GF gradients on biomaterials. This achievement is important for the improvement of interface designs that promote the integration of tissues into bone. 


\subsection{Introduction}

GFs are involved in several cellular processes, such as cell signalling, migration, survival, proliferation and differentiation [1-3]. GFs are produced and secreted by cells and either bind to target cell receptors or to GF-binding molecules that are present in the ECM [4, 5]. Their spatiotemporal presentation is crucial for the proper development and function of tissues and organs. In the Drosophila embryo, a bone morphogenetic protein (BMP) activity gradient is required for the patterning of the dorsal and lateral tissues [6], while in the developing wing of the Drosophila, a BMP activity gradient is established across the anterior/posterior (A/P) axis [7]. In growing tissues, concentration gradients of heparin-binding vascular endothelial growth factor A (VEGF-A) isoforms are crucial to guide sprouting endothelial cells in initiating blood vessel branching [8]. Presenting GFs in a spatially-controlled manner is therefore crucial to mimic the natural microenvironment that cells sense within a target tissue. Natural spatial patterning of GFs is governed by several factors, such as the production and release rate from cells, the presence of GF-binding molecules in the ECM, the degradation rate and stability [9]. GFs can be presented to target cells in a soluble or immobilized form; the later can be achieved through covalent or non-covalent binding strategies. In order to control the spatial presentation of GFs to target cells, several strategies have been exploited, such as the use of microfluidic devices; these are particularly useful for the generation of soluble gradients of GFs [10]. This strategy can be useful in the study of dose-response curves, toxicity or for the optimal concentrations of soluble GFs in target cells. One study showed that retinal progenitor cells exhibit higher mobility in the presence of low levels of epidermal growth factor (EGF) when cultured in a microfluidic device capable of generating steady-state gradients of EGF [11]. Despite the importance of evaluating the behaviour of cells towards gradients of soluble GFs, establishing steady-state gradients of soluble GFs outside a chamber of a microfluidic device is challenging. In contrast, GFs can be immobilized in a covalent or non-covalent manner, the former via direct electrostatic, hydrophobic or other secondary interactions between the GF and matrices, or interactions via other biological molecules, such as heparin, gelatin or fibronectin [9, 12]. For example, Dinis et al. (2014) developed a method of generating discontinuous concentration gradients of covalently-bound nerve growth factor (NGF) along electrospun fibres [13]. They showed that the NGF concentration gradient led to the oriented and increased growth of the dorsal root ganglion of rat neurons when compared with a single uniform distribution of NGF. In addition to the importance of the spatial presentation of GFs in tissue function, their temporal presentation is also crucial. Covalent immobilization does not offer temporal control of the GF presentation due to 
its immobilization through permanent covalent bonding. The immobilization of GFs through affinity molecules, such as heparin or hyaluronan, is a more natural way of presenting GF that allows the release and re-binding of the GF over time. Almodovar et al. (2014) developed a strategy to generate gradients of matrix-bound BMP-2 and -7 by combining poly(L-lysine) and hyaluronan in a microfluidic device [14]. With this technology, they were able to investigate the optimal concentration of matrix-bound GFs as well the presentation of multiple GFs in the differentiation of muscle cells. Here, the spatial control of the presentation of immobilized GFs as a consequence of a preestablished gradient of a GF-binding molecule onto biomaterials is investigated as an alternative strategy. This approach would have advantages for in vivo application as it would provide the tissue with a scaffold where GFs could be released and re-bound over time in a spatially-controlled manner due to the presence of a GF-binding molecule gradient. Towards this end, a strategy is developed to spatially-control the presentation of immobilized hTGF- $\beta 1$ by generating gradients of a TGF- $\beta 1$-binding peptide on PCL films. $P C L$ films were chemically modified and covalently functionalized with the TGF- $\beta 1$ binding peptides that can capture and immobilize hTGF- $\beta 1$ through non-covalent interactions. An automated dip coater was used to generate the gradients of the TGF- $\beta 1$ binding peptide on the PCL films, which was confirmed using XPS. Subsequently, we assessed whether the gradients of the TGF- $\beta 1$-binding peptide would translate to a gradient of immobilized hTGF- $\beta 1$ once incubated homogeneously with a solution of the GF. The profile of the TGF- $\beta 1$-binding peptide gradient can be tuned by controlling parameters such as dipping out speed or peptide concentration, which ultimately affect the profile of the immobilized hTGF- $\beta 1$. This technique may be useful in the establishing of gradients of bioactive molecules (GFs, peptides, ECM components) onto biomaterials to improve mimics of the natural microenvironment of tissues. This technique can be applied to a broad class of medical devices already on the market, endowing them with novel biological potential. 


\subsection{Materials and methods}

\subsubsection{Materials}

$\mathrm{N}, \mathrm{N}, \mathrm{N}^{\prime}, \mathrm{N}^{\prime}$-Tetramethyl-O-(1H-benzotriazol-1-yl)uronium hexafluorophosphate (HBTU) was obtained from MultiSynTech. Chloroform and 1-methyl-2-pyrrolidinone (NMP) were purchased from VWR Chemicals. $\mathrm{NaOH}$ was obtained from Riedel-de Haën. All other reagents or products were purchased from Sigma-Aldrich unless noted otherwise.

\subsubsection{Peptide synthesis and purification}

The TGF-ß1-binding peptide was synthetized by standard Fmoc-solid phase peptide synthesis in a Syro II MultiSynTech automated peptide synthesizer. The TGF- $\beta 1$-binding peptide KGLPLGNSH was prepared on Fmoc-Rink 4-methylbenzhydrylamine (MBHA) resin (MultiSynTech $\mathrm{GmBH}, 50 \mathrm{mg}$ scale, substitution $0.52 \mathrm{mmol} / \mathrm{g}$ ), using $0.26 \mathrm{M}$ of HBTU $0.52 \mathrm{M}$ of N,N-Diisopropylethylamine (DIPEA), $2 \mathrm{M}$ of piperidine and $0.29 \mathrm{M}$ of each amino acid. The $\mathrm{N}$-terminus of the final peptide sequence was manually acetylated in $16 \%$ acetic anhydride, 30\% DIPEA and 54\% NMP for one hour at room temperature. The peptide was cleaved from the resin and the amino acid side groups were deprotected using 95\% trifluoroacetic acid, 2.5\% triisopropylsilane and $2.5 \%$ milliQ water. The peptide was then collected by precipitation in cold diethyl ether and the organic solvents were removed in a rotatory evaporator. The peptide was redissolved in milliQ water and lyophilized overnight. The resulting product was purified using standard preparative HPLC methods. MS (ESI): $\mathrm{m} / \mathrm{z}=964.1[\mathrm{M}+\mathrm{H}]+\left(\right.$ calculated 963.1 for $\left.\mathrm{C}_{42} \mathrm{H}_{70} \mathrm{~N}_{14} \mathrm{O}_{12}\right)$ for KGLPLGNSH.

\subsubsection{Preparation of PCL films displaying TGF- $\beta 1$-binding peptides}

A $12.5 \%(\mathrm{w} / \mathrm{v})$ solution of $\mathrm{PCL}$ in chloroform was prepared and homogenized by sonication. When the solution was completely homogenized, PCL films were prepared by casting in a petri dish pre-silanized with a PFDTS $(1 \mathrm{H}, 1 \mathrm{H}, 2 \mathrm{H}, 2 \mathrm{H}$ Perfluorodecyltrichlorosilane, $\geq 97 \%, A B C R \mathrm{GmbH}$ ) anti-sticky layer. Upon solvent evaporation, the polymer was melted and allowed to again solidify. The polymer was then cut into circular films with a diameter of $21 \mathrm{~mm}$ in order to fit inside the wells of a 12-well plate. The individual circular films were extensively washed with demi-water and milliQ water and dried with a $\mathrm{N}_{2}$ stream. The dried films were exposed to oxygen plasma for $5 \mathrm{~min}$ (at an oxygen pressure of $1.0 \mathrm{bar}$, a vacuum pressure of $200 \mathrm{mbar}$ and a current of $40 \mathrm{~A}$ ) and subsequently immersed in a $1 \mathrm{M} \mathrm{NaOH}$ solution for one hour with gentle 
agitation. PCL films were then washed and dried as mentioned above and incubated with a solution of $50 \mathrm{mM}$ 1:1 NHS/EDC in MES buffer for one hour with agitation. PCL films were again washed and dried as mentioned above and incubated with $1 \mathrm{mM}$ of the peptide in phosphate buffered saline (PBS) for 4 hours with agitation. Films were then extensively washed with PBS and sterilized by incubating the films overnight in a solution of $10 \%$ penicillin/streptomycin (Life Technologies) in PBS prior to cell seeding.

\subsubsection{Generation of TGF- $\beta 1$-binding peptide gradients on PCL films}

Pellets of PCL were placed inside a mould of $6 \mathrm{~cm}$ in length and $1 \mathrm{~cm}$ in width and heated to $100^{\circ} \mathrm{C}$ for 5 min using a hot press. After cooling, the solid $\mathrm{PCL}$ sample was cut into two halves. Individual PCL films of $3 \mathrm{~cm}$ in length and $1 \mathrm{~cm}$ in width were extensively washed with demi-water and milliQ water and dried with a N2 stream. The dried films were exposed to oxygen plasma for $5 \mathrm{~min}$ (at an oxygen pressure of $1.0 \mathrm{bar}$, a vacuum pressure of $200 \mathrm{mbar}$ and a current of $40 \mathrm{~A}$ ) and subsequently immersed in a $1 \mathrm{M} \mathrm{NaOH}$ solution for one hour with gentle agitation. PCL films were then washed and dried as mentioned above, and incubated with a solution of $50 \mathrm{mM}$ 1:1 NHS/EDC in MES buffer for one hour with agitation. PCL films were then washed and dried as described above. Gradients of TGF- $\beta 1$-binding peptides were generated by dipping the PCL films in a solution of the TGF$\beta 1$-binding peptide in PBS at a controlled speed using a motorized dip coater. Films were then extensively washed with PBS and stored for further experiments.

\subsubsection{XPS measurements}

XPS spectra were measured using a Quantera scanning X-ray multiprobe instrument (Physical Electronics), equipped with a monochromatic Al Ka X-ray source operated at $1486.6 \mathrm{eV}$ and $55 \mathrm{~W}$. Spectra were referenced to the main aliphatic $\mathrm{C}$ 1s peak set at 284.8 $\mathrm{eV}$. The X-ray beam size was $200 \mu \mathrm{m}$ and the data were collected from surface areas of $100 \mu \mathrm{m} \times 300 \mu \mathrm{m}$ with a pass energy of $224 \mathrm{eV}$ and a step energy of $0.8 \mathrm{eV}$ for survey scans, at a detector input angle of $45^{\circ}$. Measurements were collected after three scanning cycles. Charge neutralization was achieved by low-energy electrons and lowenergy argon ions.

\subsubsection{TGF- $\beta 1$ binding and immunofluorescence}

The PCL films were incubated with $1 \mu \mathrm{g} / \mathrm{mL}$ of hTGF- $\beta 1$ (PeproTech) in $0.5 \%(\mathrm{v} / \mathrm{v})$ phosphate buffered saline tween-20 (PBST) for one hour with gentle agitation. The films were then washed six times for $10 \mathrm{~min}$ with $0.5 \%(\mathrm{v} / \mathrm{v})$ PBST and then with PBS alone for 
a further $10 \mathrm{~min}$. Next, the films were blocked for one hour with PBS containing $1 \%(\mathrm{w} / \mathrm{v})$ BSA. Afterwards, the films were incubated with a $5 \mu \mathrm{g} / \mathrm{mL}$ solution of the primary antibody (mouse monoclonal anti-human TGF- $\beta 1, R \& D$ systems) in the blocking solution for one hour with agitation. The films were washed three times as mentioned above and then incubated with a $4 \mu \mathrm{g} / \mathrm{mL}$ solution of the secondary antibody (goat anti-mouse Alexa Fluor 546, Invitrogen) in PBS containing 1\% (w/v) BSA for one hour with gentle agitation. Prior to fluorescence microscopy, the films were washed three times for 10 min with $0.5 \%(\mathrm{v} / \mathrm{v})$ PBST and rinsed three times with PBS.

\subsubsection{Fluorescence imaging and quantification}

The TGF- $\beta 1$ gradient was visualized using an automated fluorescence microscope (BD Pathway). Several pictures were taken along the sample, with each picture having a 10\% overlap with the next. After imaging, all pictures were mounted in a single picture that covered the complete length of the sample using the Stitching plugin from ImageJ. ImageJ was also used for quantification of fluorescence across the gradient. The values of fluorescence per pixel were then converted to fluorescence per centimetre.

\subsubsection{Statistical analysis}

The data were analysed using a Student's paired t-test, a one-way analysis of variance followed by a Tukey's multiple comparison test $(p<0.05)$ or a two-way analysis of variance. The values represent the mean and standard deviation of three independent measurements. 


\subsection{Results}

6.3.1 Amount of immobilized hTGF- $\beta 1$ is dependent on the reaction time between the PCL films and the TGF- $\beta 1$-binding peptide

We first tested whether the reaction time between the NHS groups at the surface of the PCL films and the TGF- $\beta 1$-binding peptide (Figure S6.1) would lead to differing surface concentrations of the peptide and consequently to varying amounts of immobilized hTGF- $\beta 1$. PCL films were chemically modified until the NHS/EDC step, as mentioned in chapter 3, and were then incubated without (negative control) or with the TGF- $\beta 1$ binding peptide for 10, 20, 30, 40, 50,60 and 240 (positive control) min. Next, the films were incubated with hTGF- $\beta 1$, the antibody stained against the GF, imaged and the fluorescence was quantified (Figure 6.1). Increasing the reaction time between the PCL films and the NHS groups at the surface and the TGF- $\beta 1$-binding peptide led to an increase in the amount of immobilized hTGF- $\beta 1$. Our data show that the amount of immobilized hTGF- $\beta 1$ increased linearly with the reaction time until $40 \mathrm{~min}$. After $40 \mathrm{~min}$, further increasing the reaction time did not lead to a significant increase in the fluorescence intensity. There was also no statistically significant difference observed between the results from the $30 \mathrm{~min}$ and higher reaction times; therefore, we selected $30 \mathrm{~min}$ as the optimal reaction time in order to achieve a gradient of the TGF- $\beta 1$-binding peptide on the PCL film.

A

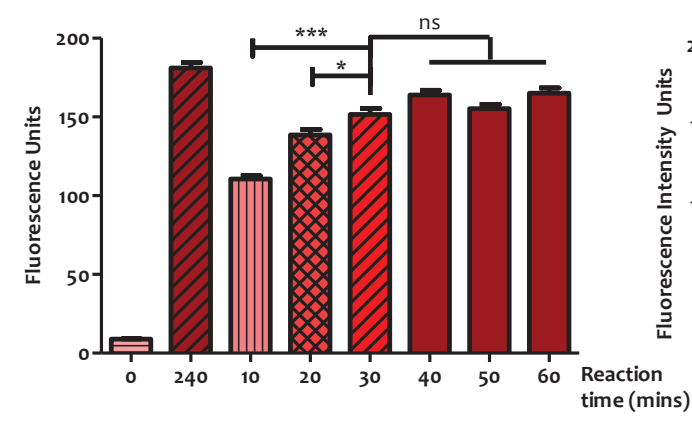

B

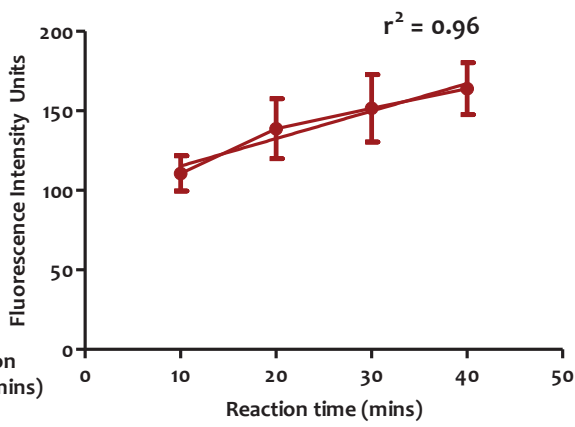

Figure 6.1. (A) Fluorescence quantification of the antibody staining assay against $h T G F-B 1$, which was immobilized on the TGF-81-binding peptide functionalized PCL films, for different reaction times. (B) Fluorescence intensity plotted vs reaction time. The data represent mean $\pm S D$ of six measurements per sample $(n=5){ }^{*} p<0.05 * * * p<0.0001$ (one-way ANOVA). 


\subsubsection{Characterization of TGF- $\beta$ 1-binding peptide gradients on PCL films}

An automated dip coater machine was used to generate gradients of the TGF- $\beta 1$-binding peptide on PCL films (Figure 6.2). The films were held vertically using a grip, immersed into the TGF- $\beta 1$-binding peptide solution and pulled out at controlled speeds. The longer a certain region of the film was allowed to react with the TGF- $\beta 1$-binding peptide solution, the higher the surface concentration of the peptide. A speed of $0.1 \mathrm{~cm} / \mathrm{min}$ was used to generate the gradients, and the gradients were subsequently validated using XPS. Five different regions of the film were scanned and five areas per region were measured (Figure 6.3). When the NHS groups at the surface of the film were replaced by the TGF$\beta 1$-binding peptide, an increase in nitrogen content and a decrease in oxygen content was observed by XPS analysis (Figure 6.4A). As we moved from the regions of the film that were in contact with the peptide solution for short periods to regions that were allowed to react for longer, an increase in the nitrogen and a decrease in the oxygen content was observed, as expected (Figure 6.4C). It appears, however, that a plateau was reached for the final three regions measured. From the nitrogen spectra, two different peaks were observed: a peak at $399 \mathrm{eV}$ that corresponded to the bonds present in the peptide chain and a peak at $402 \mathrm{eV}$ corresponding to the NHS groups (Figure 6.4B). The area of a given peak divided by the sum of the area of all peaks of the corresponding spectra gave the ratio of the type of bond present. Our data show that the area of the peak from the peptide increased when moving from regions of the PCL film with short reaction times to regions with longer reaction times, while the area from the NHS group simultaneously decreased. In summary, these results show that by using an automated dip coater and by controlling the time that a given area of the PCL film is allowed to react with the peptide solution, it is possible to generate gradients of the TGF- $\beta 1$-binding peptide on PCL films. 


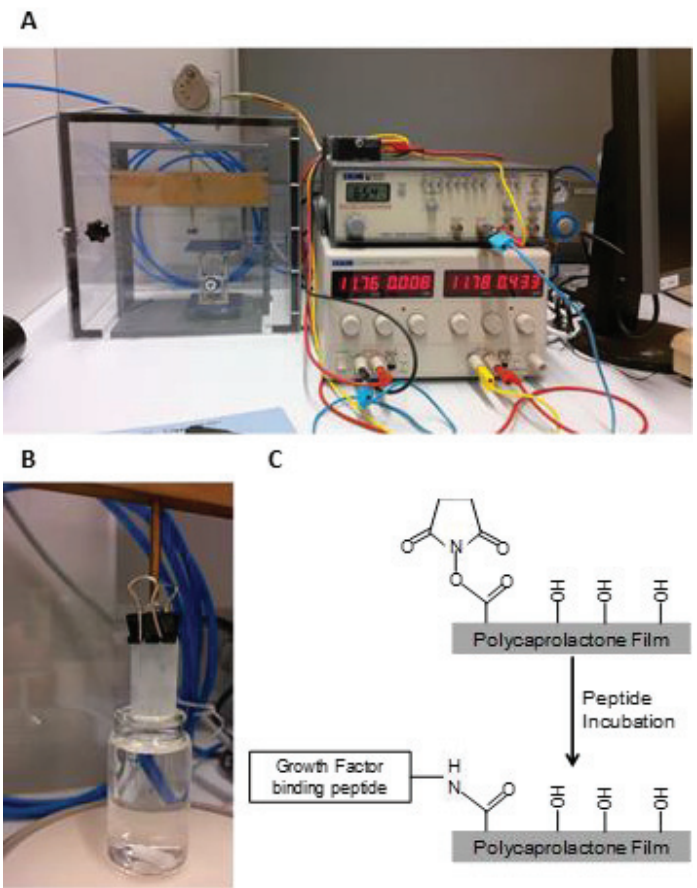

Figure 6.2. (A) Automated dip coater machine used for the generation of TGF-81-binding peptide gradients on PCL films. (B) PCL films were held vertically by the grip and immersed in a solution of the TGF-B1-binding peptide in PBS. The films were pulled out of the solution at controlled speeds. (C) Chemical modifications at the PCL film that occurred during the incubation of the films with NHS groups at the surface with the TGF-B1-binding peptide.

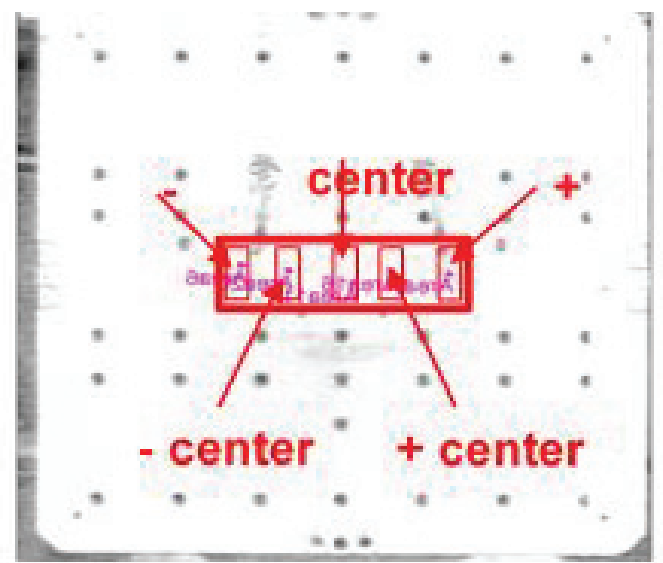

Figure 6.3. Areas of the PCL film scanned during XPS measurements. The left side corresponds to the area with low peptide concentration, and the right side with the area of high peptide concentration. 
A

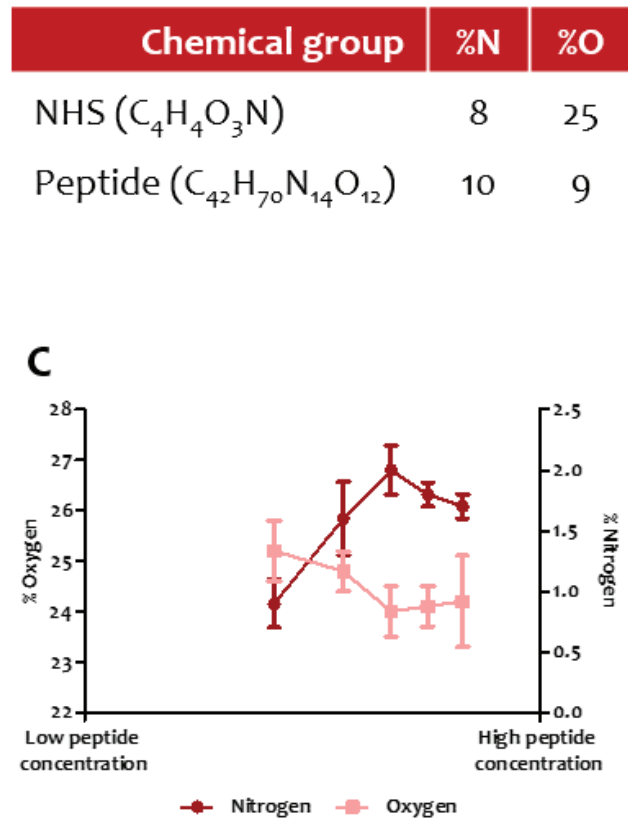

B

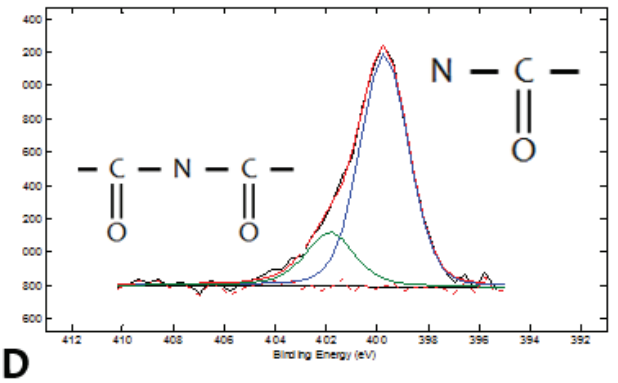

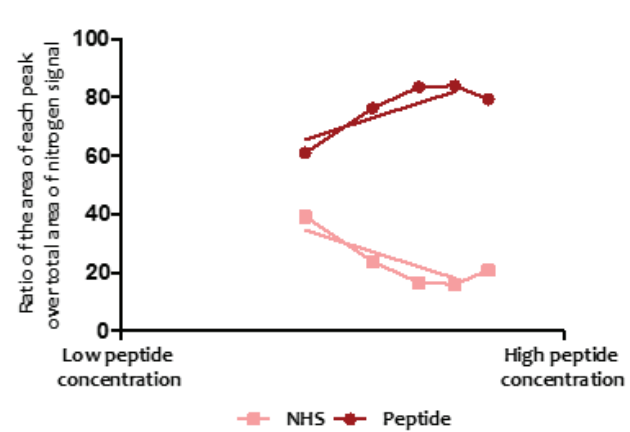

Figure 6.4. (A) The nitrogen and oxygen composition of the NHS group and the TGF-B1-binding peptide. (B) Characteristic XPS spectra of nitrogen, where two peaks are visible: at $399 \mathrm{eV}$, corresponding to the TGF-61-binding peptide, and at $402 \mathrm{eV}$, corresponding to the NHS group. (C) Atomic composition of oxygen and nitrogen along the film. (D) The ratio of the peak areas at 399 and $402 \mathrm{eV}$ divided by the total area of the nitrogen XPS spectra for the five areas of the gradient measured. The linear regression excludes the final point.

\subsubsection{TGF- $\beta 1$-binding peptide gradients lead to gradients of hTGF- $\beta 1$}

Next, the binding capacity of hTGF- $\beta 1$ to the gradients of the TGF- $\beta 1$-binding peptide was investigated via incubation with a solution of the GF. PCL films with TGF- $\beta 1$-binding peptide gradients were incubated with $1 \mu \mathrm{g} / \mathrm{mL}$ of hTGF- $\beta 1$ for one hour and then stained with the antibody against the GF. Images were recorded along the sample and mounted in one single picture that covered the entire length of the PCL film. Fluorescence intensity per pixel was calculated using ImageJ. First, we assessed whether the concentration of the peptide used to generate the gradient would influence the profile of captured hTGF- $\beta 1$. In our negative control, PCL films with NHS groups dipped into PBS, no fluorescence signal was observed (Figure 6.5A and 6.6). In contrast, an increasing profile of fluorescence intensity was observed when the TGF- $\beta 1$-binding peptide gradients were incubated with hTGF- $\beta 1$, clearly showing that gradients of hTGF- $\beta 1$ were achieved (Figure $6.5 \mathrm{~B}, \mathrm{C}$ and $\mathrm{D}$ ). The concentration of TGF- $\beta 1$-binding peptide used to 
generate the gradient influenced the profile of immobilized hTGF- $\beta 1$ (Figures $6.5 \mathrm{C}$ and 6.6). When the films were pulled out of a solution of $1 \mathrm{mM}$ of TGF- $\beta 1$-binding peptide at the speed of $0.1 \mathrm{~cm} / \mathrm{min}$, a linear increase in the fluorescence signal was observed from 0 to $1.9 \mathrm{~cm}\left(r^{2}=0.93\right)$. From 1.9 until $3.0 \mathrm{~cm}$, a plateau of fluorescence was reached. The slope for this linear region was $1.2 \pm 0.1$ fluorescence units $/ \mathrm{cm}(\mathrm{FU} / \mathrm{cm})$. Using a lower concentration, $0.5 \mathrm{mM}$ of TGF- $\beta 1$-binding peptide, but with the same pulling out speed, allowed a different profile of immobilized hTGF- $\beta 1$ to be observed (Figures 6.5B and 6.6), with a linear region only observed from 1.9 to $3.0 \mathrm{~cm}\left(r^{2}=0.94\right)$ with a slope of $1.7 \pm 0.1$ $\mathrm{FU} / \mathrm{cm}$. The pulling out speed also influenced the gradient of the TGF- $\beta 1$-binding peptide and consequently the profile of immobilized TGF- $\beta 1$. When a speed of $0.15 \mathrm{~cm} / \mathrm{min}$ was used, using $1 \mathrm{mM}$ of peptide, a linear behaviour in the fluorescence response was observed across the entire film $\left(r^{2}=0.9\right)$ with a slope of $0.63 \pm 0.0 \mathrm{FU} / \mathrm{cm}$ (Figures 6.5D and 6.7). Our data show that the gradients of TGF- $\beta 1$-binding peptide generated using an automated dip coater led to a gradient of immobilized hTGF- $\beta 1$. Parameters such as the concentration of TGF- $\beta 1$-binding peptide or the pulling out speed used affected the gradient of the TGF- $\beta 1$-binding peptide on the PCL film, ultimately influencing the profile of the immobilized hTGF- $\beta 1$.

(A)

(B)

(C)

(D)

Figure 6.5. Immunostaining against hTGF- 81 immobilized on gradients of the TGF-81-binding peptide that were generated on PCL films using: $(A)$ o $\mathrm{mM}$ of TGF- $B 1$-binding peptide and $0.1 \mathrm{~cm} / \mathrm{min}$ of pulling out speed; (B) $0.5 \mathrm{mM}$ of TGF-B1-binding peptide and $0.1 \mathrm{~cm} / \mathrm{min}$ of pulling out speed; (C) $1 \mathrm{mM}$ of TGFB1-binding peptide and $0.1 \mathrm{~cm} / \mathrm{min}$ of pulling out speed; and (D) $1 \mathrm{mM}$ of TGF-B1-binding peptide and $0.15 \mathrm{~cm} / \mathrm{min}$ of pulling out speed. 


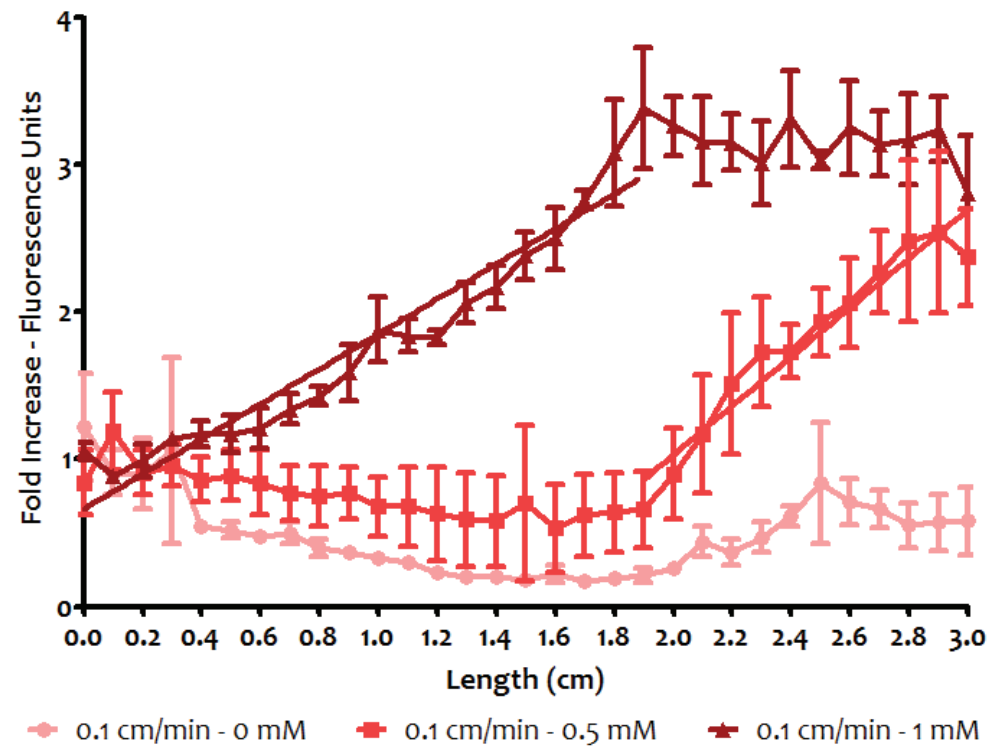

Figure 6.6. Profiles of the immobilized TGF-B1 on TGF-B1-binding peptide gradient generated using different concentrations of the peptide at the speed of $0.1 \mathrm{~cm} / \mathrm{min}$, as a function of the length of the $P C L$ films. Fluorescence was calculated using ImageJ.

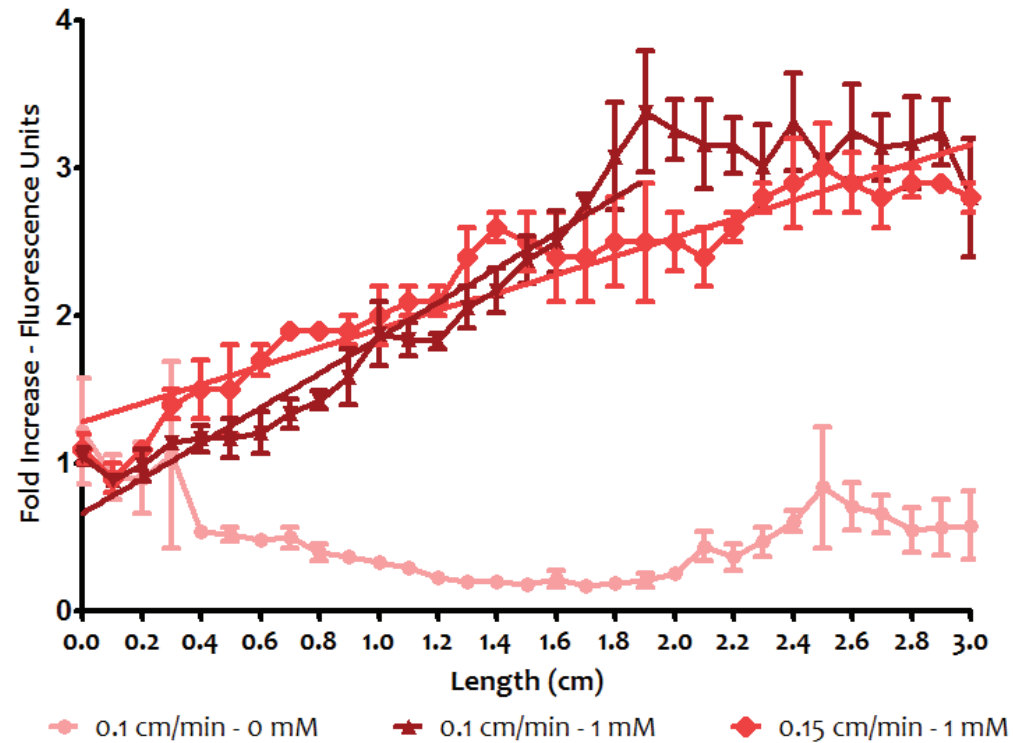

Figure 6.7. Profiles of the immobilized TGF- $B 1$ on TGF- 81 -binding peptide gradients generated using 1 $\mathrm{mM}$ of TGF-81-binding peptide at different pulling out speeds, as a function of the length of the PCL films. Fluorescence was calculated using ImageJ. 


\subsection{Discussion}

Several approaches using cells, biomaterials and bioactive molecules have been previously investigated to engineer tissue constructs [15-17]. However, less attention has been paid to the interfaces that connect distinct tissues. This is particularly important in musculoskeletal injuries, as body motion requires a synchronized interaction between distinct tissues and the integration of bone with soft tissues, such as tendons, ligaments and cartilage. Tendons and ligaments ( $\mathrm{T} / \mathrm{L}$ ) transfer force to and from the skeletal system and, by distributing the loads applied, movement is generated [18]. They attach to the bone in a zone called the enthesis or insertion site [19]. This heterogeneous interface is characterized by gradients of tissue organization, composition, cell type, signalling cues and mechanical properties, which function together to effectively transfer stress between mechanically-distinct materials (soft tissue and bone) and sustain the homeostasis of the interface [20]. Due to its critical role in body biomechanics, several orthopaedic injuries require the repair of a ruptured $T / L$ to its bone insertion, which in most cases is not reestablished [21]. Therefore, it is not only important to design strategies that can heal and regenerate individual tissues such as bone and $\mathrm{T} / \mathrm{L}$, but it is also crucial to consider the biomechanical and cellular composition of the insertion zone in the design of such therapies [22]. The use of different materials, the incorporation of multiple cell types and the spatial presentation of bioactive factors are aspects that must be taken into consideration in the design of a scaffold for the healing and regeneration of a tissue interface. Therefore, the ideal scaffold should support the growth and differentiation of different cell types, direct their cellular communications and promote the formation of a controlled heterogeneous matrix [23]. Spalazzi et al. (2008) created a mechanoactive scaffold to engineer the insertion zone by combining different ratios of poly(lactic-co-glycolic acid) (PLGA) and bioactive glass [24]. They showed that such a scaffold led to the upregulation of fibrocartilage markers and the potential to establish fibrocartilage on tendon-based grafts. In other work, the same author developed a triphasic scaffold (one for ligament formation, one for the interface and one for the bone region) [25]. When cultured with fibroblasts, chondrocytes and osteoblasts, the triphasic scaffold supported multilineage cellular interactions, tissue infiltration and matrix production in a subcutaneous athymic rat model. They also observed the formation of mineral and fibrocartilage-like tissue regions in the tri-cultured group. He et al. (2012) showed that culturing mesenchymal stem cells between fibroblasts and osteoblasts on a hybrid scaffold (silk for the ligament region and hydroxyapatite for bone), led to their differentiation into fibrocartilage [26]. The strategies mentioned above are based on stratified scaffolds and have been designed to mimic the transition between different 
tissues. Due to the heterogeneous structural organization in interfaces and the need to support different cell phenotypes, a new field has recently emerged based on the design of scaffolds with property gradients, such as mechanical or chemical factors. In contrast to the previously presented studies, this approach allows the precise control of the gradual and continuous transition of properties [23]. Using an extrusion/electrospinning process, Eriksen et al. (2008) created gradients of tricalcium phosphate nanoparticles along PCL mesh [27]. They seeded mouse preosteoblast cells (MC3T3-E1) on the scaffold and showed that after four weeks there was a formation of a continuous gradient in extracellular matrix, including collagen and mineral content, reflecting the gradient of the calcium nanoparticles. In another study, Li et al. (2009) formed mineral gradients along electrospun PLGA nanofibers [28]. The distribution of the mineral along the fibres led to a gradient of stiffness that affected the number and the osteogenic response of $\mathrm{MC}_{3} \mathrm{~T}_{3}$ cells. The changes in structure, composition and organization in tissues is correlated with spatial changes in biological factors [29]. More recently, Di Luca et al. (2016) developed a scaffold with a pore shape gradient. They showed that within the same scaffold, square pores better supported chondrogenic differentiation, whereas rhomboidal pores enhanced the osteogenic differentiation of mesenchymal stem cells (MSCs) [30]. Philips et al. (2008) showed that by generating gradients of retrovirus encoding the osteogenic transcription factor Runx2 on collagen scaffolds, it was possible to control the spatial distribution of osteoblastic differentiation and deposition of the mineralized matrix of seeded fibroblasts [31]. This correlation between Runx2 and osteogenic activity was maintained in vivo. Other bioactive factors of interest are GFs, since they are involved in several cellular processes and their spatial presentation is crucial for tissue development and healing. One of the most important classes of GFs is the transforming growth factor $\beta$ (TGF $\beta$ ) family. In tendons, TGF $\beta$ is active during all stages of healing and is involved in the regulation of cell migration, collagen production, cell proliferation and cell-matrix interactions $[32,33]$. Of the three isoforms, TGF- $\beta 1$ and $\beta_{3}$ are the most important in the growth and differentiation of the musculoskeletal system [11]. Kuo et al. (2008) demonstrated distinct spatiotemporal protein expression patterns of TGF $\beta$ s and their receptors with respect to ECM components (collagen type III, decorin and fibronectin) at the midsubstance and myotendinous junction (MTJ) of developing chick tendons [34]. Due to the role of TGF- $\beta 1$ and the importance of controlling its spatial distribution, we developed a strategy to generate gradients of immobilized hTGF- $\beta 1$ on biomaterials to improve the healing and regeneration of soft tissue and insertion zones. In this work, we functionalized PCL films with a TGF- $\beta 1$-binding peptide in order to non-covalently immobilize hTGF- $\beta_{1}$ on the material via affinity interaction with the peptide. The peptide was previously described by Shah et al. (2009) 
[35]. By dipping the PCL film into a solution of the TGF- $\beta 1$-binding peptide using an automated dip coater, we were able to generate TGF- $\beta 1$-binding peptide gradients on the surface of the film, which once incubated homogenously with hTGF- $\beta 1$ led to a gradient of the immobilized GF.

A gradient of a GF has been achieved due to the presence of a gradient of a GF-binding molecule on a biomaterial, which has not previously been reported for hTGF- $\beta 1$. Generating a gradient of a GF-binding molecule has several advantages over the strategies that generate gradients of GFs. Gradients of GFs are usually achieved through strategies that require covalent immobilization of the GF on the biomaterial [36]. This strategy does not allow a homogeneous presentation of GF due to the multiple groups present in the GF that can be involved in the chemical reaction required for immobilization [9]. Additionally, the bioactivity of the GF may be lost during the immobilization process due to conformational changes in the structure of the GF or impedance of the epitopes of the GF that bind to the respective receptors. Finally, once the covalent bond between the GF and the biomaterial is broken, the GF gradient will be lost. In summary, although being able to generate GF gradients, the disadvantages of this strategy are associated with poor bioactivity and no temporal control over the presentation of the GF. The bioactivity-associated problems can be overcome by immobilizing GFs through non-covalent interactions. ECM components, such as heparin, have the ability to bind and then release the GF in a sustained manner [37]. Generating gradients on biomaterials presenting these GF-binding molecules can be achieved using dipping techniques or with microfluidic devices, and will minimize the problems associated with bioactivity loss. However, as mentioned above, once the GF is release from the GF-binding molecules, the integrity of the gradient is compromised. Additionally, due to a uniform distribution of GF-binding molecules on the biomaterial, the gradient of the GF cannot be reestablished. To overcome this, a GF-binding molecule gradient can be advantageous over a GF gradient. The GF-binding molecules can bind and release the GF, securing the integrity of a dynamic gradient. The integrity of the GF gradient will only be dependent on the degradation rate of the biomaterial. Here, we showed that generating a TGF- $\beta$ 1-binding peptide gradient on a biomaterial led to a gradient of immobilized hTGF- $\beta 1$. The spatial distribution of the immobilized hTGF- $\beta 1$ was dictated by the profile of the gradient of the TGF- $\beta$ 1-binding peptide, which can be adjusted by controlling parameters such as the peptide concentration to establish the gradient or the dipping out speed. This work shows for the first time an alternative approach for the generation of GF gradients on biomaterials, revealing new strategies 
for the design of novel biomaterials for the regeneration of heterogeneous tissues and the interface between multiple tissues.

\subsection{Conclusions}

In this work, we demonstrated a strategy to spatially control the presentation of hTGF$\beta 1$ in biomaterials. This was accomplished by generating gradients of TGF- $\beta 1$-binding peptide on PCL films by using an automated dip coater. The profile of the TGF- $\beta 1$-binding peptide gradient can be adjusted by altering parameters such as the peptide concentration or dipping out speed, ultimately leading to the precise control over the presentation of immobilized hTGF- $\beta 1$. The technique demonstrated here can be used in the design of biomaterials with bioactive molecule gradients, such as GFs, peptides or other molecules, allowing the precise spatial control of the bioactive molecule, in order to better mimic the microenvironment sensed by cells and tissues.

\subsection{Acknowledgments}

We acknowledge Smith\&Nephew for providing financial support for this project, and Marcel de Bruine for providing support for use of the dip coater device. 


\subsection{References}

1. Lind M, Growth factors: possible new clinical tools. A review. Acta Orthop Scand, 1996. 67(4): p. 407-17.

2. Barrientos S, et al., Growth factors and cytokines in wound healing. Wound Repair Regen, 2008. 16(5): p. 585-601.

3. Werner $\mathrm{S}$ and Grose R, Regulation of wound healing by growth factors and cytokines. Physiol Rev, 2003. 83(3): p. 835-70.

4. Mclnnes $C$ and Sykes BD, Growth factor receptors: structure, mechanism, and drug discovery. Biopolymers, 1997. 43(5): p. 339-66.

5. Ashikari-Hada $\mathrm{S}$, et al., Characterization of growth factor-binding structures in heparin/heparan sulfate using an octasaccharide library. J Biol Chem, 2004. 279(13): p. 12346-54.

6. O'Connor MB, et al., Shaping BMP morphogen gradients in the Drosophila embryo and pupal wing,. Development, 2006.133(2): p. 183-93.

7. Ramel MC and Hill CS, The ventral to dorsal BMP activity gradient in the early zebrafish embryo is determined by graded expression of BMP ligands. Dev Biol, 2013. 378(2): p. 17082.

8. Ruhrberg C, et al., Spatially restricted patterning cues provided by heparin-binding VEGF-A control blood vessel branching morphogenesis. Genes Dev, 2002. 16(20): p. 2684-98.

9. Lee K, Silva EA, and Mooney DJ, Growth factor delivery-based tissue engineering: general approaches and a review of recent developments. J R Soc Interface, 2011. 8(55): p. 153-70.

10. Tehranirokh $\mathrm{M}$, et al., Microfluidic devices for cell cultivation and proliferation. Biomicrofluidics, 2013. 7(5).

11. Unachukwu UJ, et al., Microfluidic Generated EGF-Gradients Induce Chemokinesis of Transplantable Retinal Progenitor Cells via the JAK/STAT and PI3Kinase Signaling Pathways. PLoS One, 2013. 8(12).

12. Cabanas-Danés J, Huskens J, and Jonkheijm P, Chemical strategies for the presentation and delivery of growth factors. J. Mater. Chem. B, 2014. 2(17): p. 2381-2394.

13. Dinis TM, et al., Method to form a fiber/growth factor dual-gradient along electrospun silk for nerve regeneration. ACS Appl Mater Interfaces, 2014. 6(19): p. 16817-26.

14. Almodóvar J, et al., Spatial patterning of BMP-2 and BMP-7 on biopolymeric films and the guidance of muscle cell fate. Biomaterials, 2014. 35(13): p. 3975-85.

15. Langer R and Vacanti JP, Tissue engineering. Science, 1993. 260(5110): p. 920-6.

16. Griffith LG and Naughton G, Tissue engineering--current challenges and expanding opportunities. Science, 2002. 295(5557): p. 1009-14.

17. Howard D, et al., Tissue engineering: strategies, stem cells and scaffolds. J Anat, 2008. 213(1): p. 66-72.

18. Benjamin $\mathrm{M}$, et al., Where tendons and ligaments meet bone: attachment sites ('entheses') in relation to exercise and/or mechanical load. J Anat, 2006. 208(4): p. 471-490.

19. Apostolakos J, et al., The enthesis: a review of the tendon-to-bone insertion. Muscles Ligaments Tendons J, 2014. 4(3): p. 333-42.

20. Lu HH and Thomopoulos S, Functional attachment of soft tissues to bone: development, healing, and tissue engineering. Annu Rev Biomed Eng, 2013. 15: p. 201-26.

21. Lui PPY, et al., Biology and augmentation of tendon-bone insertion repair. J Orthop Surg Res, 2010.5(59). 
22. Yang PJ and Temenoff JS, Engineering orthopedic tissue interfaces. Tissue Eng Part B Rev, 2009. 15(2): p. 127-41.

23. Lu HH, et al., Tissue engineering strategies for the regeneration of orthopedic interfaces. Ann Biomed Eng, 2010. 38(6): p. 2142-54.

24. Spalazzi JP, et al., Mechanoactive scaffold induces tendon remodeling and expression of fibrocartilage markers. Clin Orthop Relat Res, 2008. 466(8): p. 1938-48.

25. Spalazzi JP, et al., In vivo evaluation of a multiphased scaffold designed for orthopaedic interface tissue engineering and soft tissue-to-bone integration. J Biomed Mater Res A, 2008. 86(1): p. 1-12.

26. He $\mathrm{P}$, et al., In Vitro Ligament-Bone Interface Regeneration Using a Trilineage Coculture System on a Hybrid Silk Scaffold. Biomacromolecules, 2012. 13(9): p. 2692-703.

27. Erisken C, Kalyon DM, and Wang $\mathrm{H}$, Functionally graded electrospun polycaprolactone and beta-tricalcium phosphate nanocomposites for tissue engineering applications. Biomaterials, 2008. 29(30): p. 4065-73.

28. Li X, et al., Nanofiber Scaffolds with Gradations in Mineral Content for Mimicking the Tendon-to-Bone Insertion Site. Nano Lett, 2009. 9(7): p. 2763-8.

29. Smith L, et al., Tissue Engineering Strategies for the Tendon/ligament-to-bone insertion. Connect Tissue Res, 2012. 53(2): p. 95-105.

30. Di Luca A, et al., Tuning Cell Differentiation into a 3D Scaffold Presenting a Pore Shape Gradient for Osteochondral Regeneration. Adv Healthc Mater, 2016. 5(14): p. 1753-63.

31. Phillips JE, et al., Engineering graded tissue interfaces. PNAS, 2008. 105(34): p. 12170-12175.

32. Molloy $\mathrm{T}$, Wang $\mathrm{Y}$, and Murrell $\mathrm{G}$, The roles of growth factors in tendon and ligament healing. Sports Med, 2003. 33(5): p. 381-94.

33. James $\mathrm{R}$, et al., Tendon: biology, biomechanics, repair, growth factors, and evolving treatment options. J Hand Surg Am, 2008. 33(1): p. 102-12.

34. Kuo CK, Petersen BC, and Tuan RS, Spatiotemporal protein distribution of TGF-Bs, their receptors, and extracellular matrix molecules during embryonic tendon development. Dev Dyn, 2013. 237(5): p. 1477-1489.

35. Sahah RN, et al., Supramolecular design of self-assembling nanofibers for cartilage regeneration. PNAS, 2009. 107(8): p. 3293-3298.

36. Masters KS, Covalent growth factor immobilization strategies for tissue repair and regeneration. Macromol Biosci, 2011. 11(9): p. 1149-63.

37. Olwin BB, Heparin-binding growth factors and their receptors. Cytotechnology, 1989. 2(4): p. 351-65. 


\subsection{Supporting information}

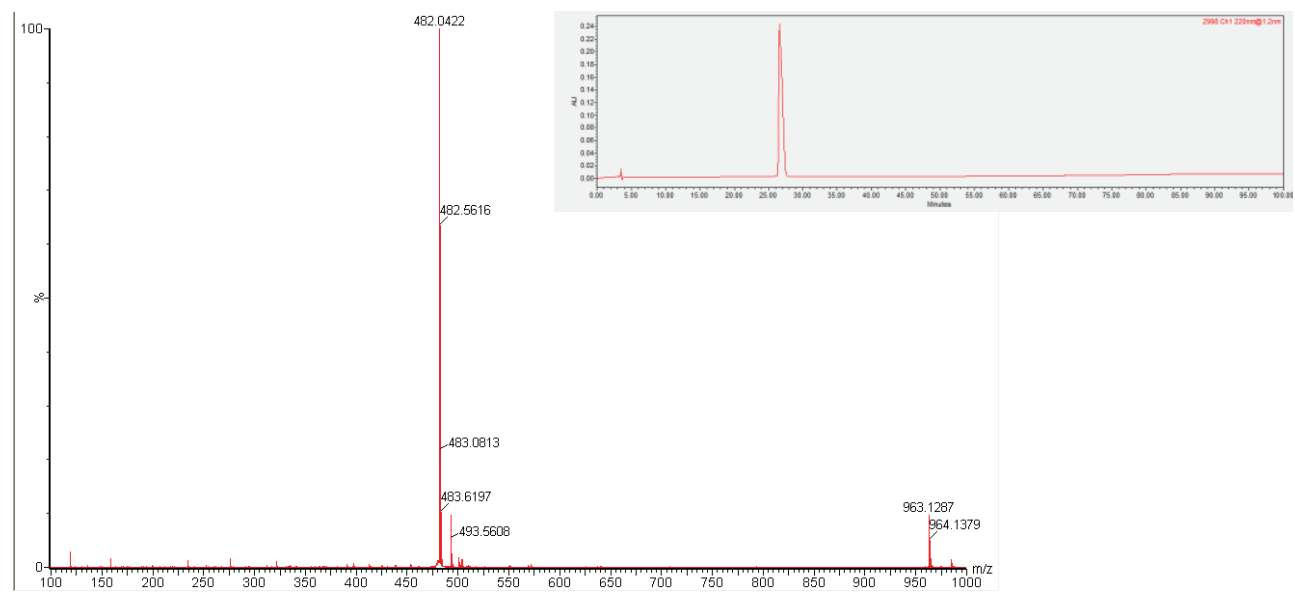

Figure S6.1. Mass spectroscopy of the TGF-81-binding peptide after purification and respective analytical HPLC. 
128 


\section{Chapter 7}

\section{Final conclusions and future perspectives}

The work described in this thesis can be exploited to capture and present endogenous growth factors (GFs) through functionalizing biomaterials with GF-binding peptides. Future perspectives regarding such strategies are discussed in the following chapter, with an eye towards enhance tendon/ligament ( $T / L)$ healing. 


\subsection{Final conclusions}

Although current surgical procedures provide good clinical outcomes, they still fail to restore the original biomechanical properties of damaged T/L. In addition, there is a considerable loss of time and function in the perioperative phase. With the goal of enhancing both reconstruction and repair of damaged $T / L$, a strategy has here been developed to capture endogenous GFs and present them to target cells and tissues. This was achieved by covalently functionalizing biomaterials with GF-binding peptides in order to capture the corresponding GF. This work focused on three particular GFs known to play key roles in $\mathrm{T} / \mathrm{L}$ - as well as in other tissues - healing: transforming growth factor $\beta 1$ (TGF- $\beta$ 1) for soft tissue, bone morphogenetic protein 2 (BMP-2) for bone, and vascular endothelial growth factor (VEGF) for vascularization. The affinity of the peptides towards the GFs is dictated by the amino acid sequence. The binding peptides were synthesized and the affinity towards the respective GF confirmed by immunofluorescence. The captured GFs retained their bioactivity as they activated the respective signalling pathways. Moreover, immobilized TGF- $\beta 1$ promoted the synthesis of collagen by human-derived hamstring cells, immobilized BMP-2 induced the synthesis of alkaline phosphatase by $\mathrm{C}_{2} \mathrm{C} 12$, while immobilized VGEF promoted the survival and proliferation of human umbilical vein endothelial cells (HUVECs). In vivo studies showed that implants functionalized with the respective GF-binding peptide captured the corresponding endogenous GF, leading to its accumulation around the implant and in turn eliciting the expected biological response. The endogenous, captured TGF- $\beta 1$ led to the more pronounced recruitment of inflammatory cells, a fibrotic response and neovascularization around the implant. The implant functionalized with the BMP-2binding peptide led to a higher matrix deposition when compared with the control, while the implants functionalized with the VEGF-binding peptide enhanced neovascularization at day 3, which ultimately led to the higher organization of the tissue around the functionalized implant at day 7 . In this work, a strategy was developed focusing on the repair of the interface between soft tissue and bone by generating gradients of GFbinding peptides. These gradients led to the spatial control of the captured GF on the biomaterial. The work in this thesis highlights the potential for the use of GF-binding peptides in the design and improvement of biomaterials by capturing endogenous GFs and allowing their presentation to target tissues. More complex strategies using the technology here developed can be explored in the future, and are briefly discussed below. 


\subsection{Future perspectives}

\subsubsection{Growth factor combination}

GFs are known to synergistically interact with other GFs and potentiate their effects [1, 2]. The combination of different GF-binding peptides onto biomaterials could potentially enhance tissue healing, making this an appealing strategy to translate to a clinical application. Murphy et al. (2014) showed that the combination of TGF- $\beta 1$ and BMP-2 upregulated the expression of chondrogenic genes in human articular chondrocytes [3]. Tachi et al. (2011) showed that TGF- $\beta 1$ enhances BMP-2-induced ectopic bone formation [4]. They showed that collagen sponges containing both TGF- $\beta 1$ and BMP-2 completely filled bone defects, created in mouse calvaria, with orthotopic bone, whereas collagen sponges with BMP-2 alone led to an only partial filling of the defects. The combination of VEGF and BMP-2 has also been reported to have a synergistic effect in bone regeneration [5]. The combination of TGF- $\beta 1$ and a BMP-2-binding peptide may be beneficial for the soft tissue in $\mathrm{T} / \mathrm{L}$ reconstruction, while for the bone, a combination of the VEGF-binding peptide with the BMP-2-binding peptide could potentially enhance bone formation.

\subsubsection{Spatial presentation of growth factors}

GFs regulate the healing process in spatial and temporal manners [6]. Due to the heterogeneity of tissues, control of the spatial presentation of GFs is crucial in order to engineer the microenvironment of tissue constructs. Controlling the spatial distribution of the GF-binding peptides onto biomaterials will eventually lead to the spatial control of the presentation of the respective GFs, allowing the design of biomaterials that could present a specific GF to a specific target location. We envision that a sleeve functionalized at the ends with a BMP-2-binding peptide and in the middle with a TGF- $\beta$ 1binding peptide would allow the healing of both bone and soft tissue during $\mathrm{T} / \mathrm{L}$ reconstruction. If wrapped around a tendon graft, the ends of the biomaterial (those functionalized with a BMP-2-binding peptide) would match the dimensions of the bone tunnels, there enhancing bone formation, while the middle part (functionalized with a TGF- $\beta 1$-binding peptide) would cover the soft tissue and promote collagen production (Figure 7.1). Such a design could be achieved through the use of dip coating, where different areas of the biomaterial are submerged into different peptide solutions, or through microfluidics [7], inkjet printing [8], lithography [9] or microprinting [10] 
techniques. This strategy would result in a biomaterial stratified with different GF peptides.

More attractive is the design of biomaterials with GF-binding peptide gradients. In chapter 6, a strategy was developed to generate gradients of TGF- $\beta 1$-binding peptide that ultimately led to a gradient of immobilized TGF- $\beta 1$. The design of biomaterials with dual gradients of GF-binding peptides would allow the complex engineering of tissue interfaces. We envision that a dual, oppositely directed, gradient of TGF- $\beta 1$ - and BMP-2binding peptides would allow the engineering of the interface between soft tissue and bone (Figure 7.2). This would lead to an accumulation of BMP-2 at one end, directing cells towards bone production, and to the accumulation of TGF- $\beta 1$ at the opposite end, directing cells towards collagen production. The combined spatial presentation of both GFs could led to the appearance of mineralized and non-mineralized fibrocartilage in the middle section of the biomaterial. Such a design could potentially promote the integration of the soft tissue with the bone. A dip coating technique could be used to generate the dual gradient. In this technique, the biomaterial is dipped into and then lifted from a peptide solution, flipped and then dipped into and lifted from the solution of the second peptide. Almodóvar et al. (2014) developed a strategy to generate gradients of immobilized BMP-2 and BMP-7 on biopolymeric films with a microfluidic device [11]. This strategy could also be employed to generate the dual gradient of GFbinding peptides. We foresee that the dip coating technique, given its simplicity, could be easily implemented in a clinical scenario, making it more attractive for commercial use than the above-mentioned alternatives. Enhancing vascularization and neurogenesis is also crucial for a proper healing response. The incorporation of peptides with an affinity for GFs, such as VEGF, neurotrophin-3 (NT-3) and fibroblast growth factor-2 (FGF-2), should also be considered in the design of the abovementioned systems.

Materials functionalized with the GF-binding peptides could also be incubated with the respective GFs and be used as delivery systems for in vivo applications. The direct incubation of the functionalized material with the GF is not currently possible due to legal restrictions regarding the use of GFs for clinical applications. However, their combination with platelet rich plasma (PRP) could be an alternative option. The material functionalized with the desired GF-binding peptide could be incubated with the patient's PRP to selectively immobilize the desired GF. The amount of captured GF could here be controlled by tuning the surface density of the GF-binding peptide. This strategy could potentiate the beneficial effects of PRP therapy and reduce its variability due to batch variation and GF concentration. 

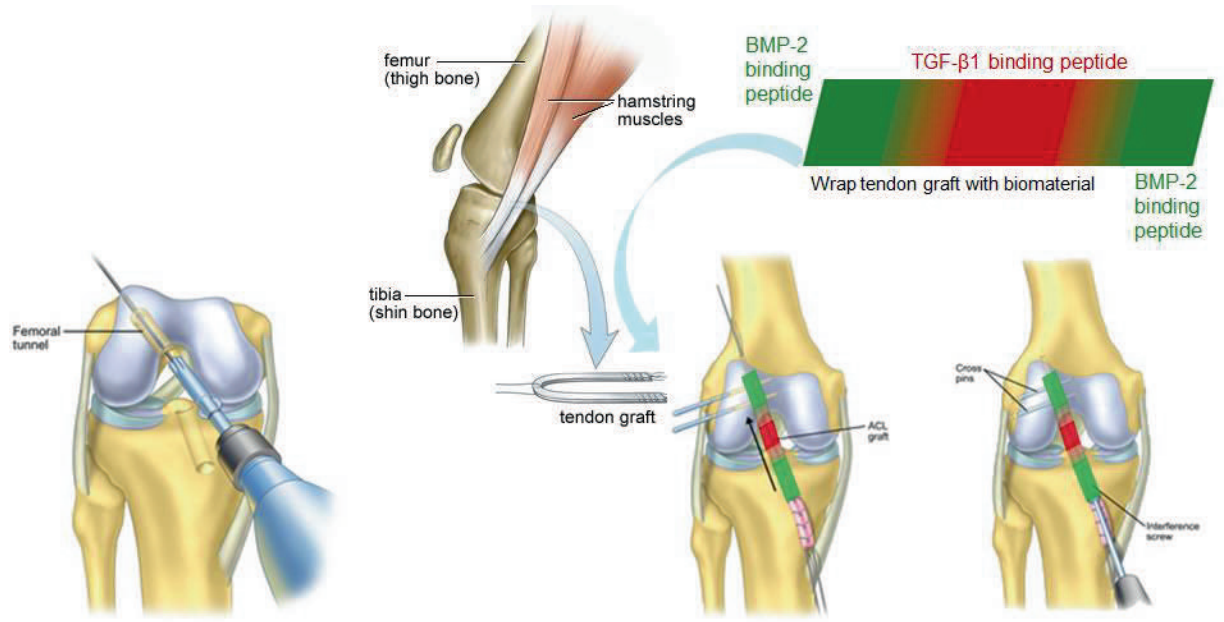

Figure 7.1. A schematic representation of the use of a biomaterial, showing patterning of GFbinding peptides in $\mathrm{ACL}$ reconstruction.

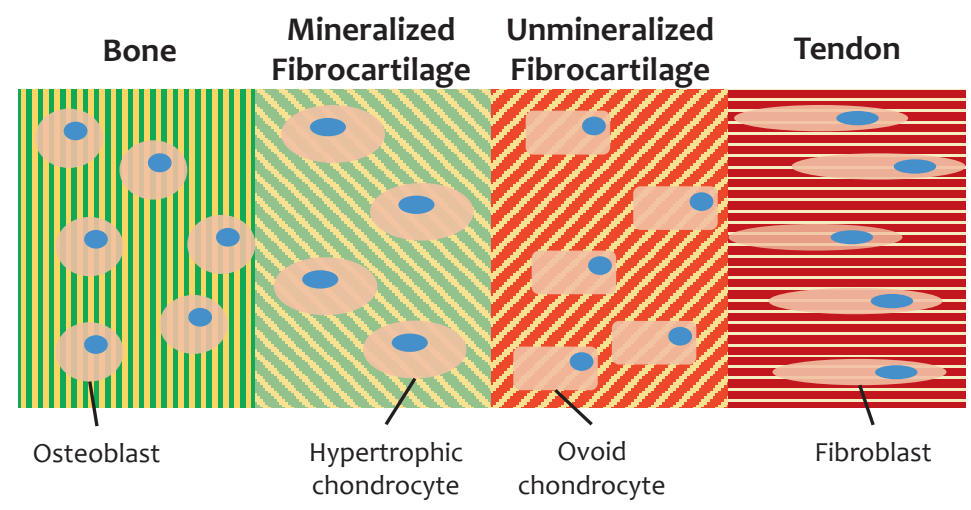

BMP-2 chondrocyte

TGF- $\beta 1$

binding peptide

\section{Biomaterial}

Figure 7.2. A schematic representation of the use of a biomaterial, displaying a dual gradient of GFbinding peptides in $A C L$ reconstruction. 


\subsubsection{Final considerations}

However, in addition to appreciating the potential to use the strategy here developed for T/L healing, other aspects must also be taken into consideration in the design of the ideal biomaterial. The suitable biomaterial should be biocompatible, biodegradable, have mechanical properties as close as possible to the native tissue, have the appropriate architecture, be possible to sterilize using standard clinical procedures without affecting the mechanical properties, and the fabrication method should be cost effective and be possible to scale up [12]. Parameters such as stiffness, ultimate tensile strength, porosity, roughness and surface chemistry should be considered when choosing the appropriate material. Additionally, the use of different materials within the same scaffold could mimic the structure of the interface between soft tissue and bone [13]. The chemical strategy used to covalently immobilize the GF-binding peptides must not change the mechanical properties, so that it does not compromise the in vivo performance of the biomaterial. Inclusion of cells on biomaterials is known to promote tissue integration of the implanted biomaterial [14]. Fibroblasts for soft tissue, osteoblasts for bone and chondrocytes for fibrocartilage can be seeded onto scaffolds to enhance tissue healing [15-17]. Co-culture systems of these cell types within the same scaffold have proven to be a valuable strategy to mimic the interface between soft tissue and bone [18]. The ideal biomaterial for $\mathrm{T} / \mathrm{L}$ should combine all of the aspects mentioned above in order to promote and accelerate the healing of injured $\mathrm{T} / \mathrm{L}$ and ultimately rescue the biomechanical properties of those tissues. We should also be mindful of and examine any changes to the established biomechanical properties of the existing grafts after applying our innovative GF capture strategy, since an interaction may occur. Thus, basic testing must be repeated after the proposed surface modifications to ensure proper and improved function. The work presented in this thesis provides a solid basis for the design of such an ideal biomaterial for $\mathrm{T} / \mathrm{L}$ healing. 


\subsection{References}

1. Pepper MS, et al., Potent synergism between vascular endothelial growth factor and basic fibroblast growth factor in the induction of angiogenesis in vitro. Biochem Biophys Res Commun, 1992. 189(2): p. 824-31.

2. Goto F, et al., Synergistic effects of vascular endothelial growth factor and basic fibroblast growth factor on the proliferation and cord formation of bovine capillary endothelial cells within collagen gels. Lab Invest, 1993. 69(5): p. 508-17.

3. Murphy MK, et al., TGF-B1, GDF-5, and BMP-2 stimulation induces chondrogenesis in expanded human articular chondrocytes and marrow-derived stromal cells. Stem Cells, 2015. 33(3): p. 762-73.

4. Tachi K, et al., Enhancement of bone morphogenetic protein-2-induced ectopic bone formation by transforming growth factor-81. Tissue Eng Part A, 2011. 17(5-6): p. 597-606.

5. Zhang W, et al., VEGF and BMP-2 promote bone regeneration by facilitating bone marrow stem cell homing and differentiation. Eur Cell Mater, 2014. 15(27): p. 1-12.

6. James R, et al., Tendon: biology, biomechanics, repair, growth factors, and evolving treatment options. J Hand Surg Am, 2008. 33(1): p. 102-12.

7. Delamarche E, et al., Patterned delivery of immunoglobulins to surfaces using microfluidic networks. Science, 1997. 276(5313): p. 779-81.

8. Delaney. Jr JT, Smith PJ, and Schubert US, Inkjet printing of proteins. Soft Matter, 2009. 5(24): p. 4866-4877.

9. Kane RS, et al., Patterning proteins and cells using soft lithography. Biomaterials, 1999. 20(23-24): p. 2363-76.

10. Delehanty JB and L. FS, Method for printing functional protein microarrays. Biotechniques, 2003. 34(2): p. 380-5.

11. Almodóvar J, et al., Spatial patterning of BMP-2 and BMP-7 on biopolymeric films and the guidance of muscle cell fate. Biomaterials, 2014. 35(13): p. 3975-85.

12. O'Brien FJ, Biomaterials \& scaffolds for tissue engineering. Mater Today, 2011. 14(3): p. 8895.

13. Spalazzi JP, et al., In vivo evaluation of a multiphased scaffold designed for orthopaedic interface tissue engineering and soft tissue-to-bone integration. J Biomed Mater Res A, 2008. 86(1): p. 1-12.

14. Howard D, et al., Tissue engineering: strategies, stem cells and scaffolds. J Anat, 2008. 213(1): p. 66-72.

15. Kiapour AM and Murray MM, Basic science of anterior cruciate ligament injury and repair. Bone Joint Res, 2014. 3(2): p. 20-31.

16. Clarke B, Normal Bone Anatomy and Physiology. Clin J Am Soc Nephrol, 2008. 3(3): p. S131S139.

17. Benjamin M and Ralphs JR, Biology of fibrocartilage cells. Int Rev Cytol, 2004. 233: p. 1-45.

18. He P, et al., In Vitro Ligament-Bone Interface Regeneration Using a Trilineage Coculture System on a Hybrid Silk Scaffold. Biomacromolecules, 2012. 13(9): p. 2692-703. 
136 


\section{Summary}

Orthopaedic injuries are associated with pain, disability, and a great financially burden for the patient and society. Tendons and ligaments are poorly vascularised and therefore have a low healing capacity. Surgical intervention is the most common therapy to direct the natural healing process of these tissues upon laceration or rupture. In some cases, however, damage exceeds the natural ability to repair even after surgical intervention. This will lead to a healed tissue whose biomechanical properties do not match the ones of the native tissue. In order to advance the medical devices implanted during surgical intervention beyond the current state of the art, knowledge from different areas of expertise, such as chemistry, surface and interface chemistry, cell biology, biomedical engineering, and clinical practice, need to be integrated to arrive at newly developed implants that integrate strategies to deliver growth factors. These growth factors are crucial to improve the integration of implants into tissue. Growth factors are also involved in the activation and regulation of several cellular functions during all stages of the healing process. Therefore, delivery systems that allow control over the spatial and temporal presentation of growth factors to the injured tissue are essential in the development of strategies to improve the healing process.

This thesis describes a new strategy to immobilise and deliver growth factors. Various biomaterials have been chemically modified and functionalised with synthetic peptides, which specifically interact with different growth factors depending on the amino acid sequence of the peptides. Peptides that interact with TGF- $\beta 1$, BMP-2, and VEGF have been synthetised and covalently immobilised onto biomaterials. The interaction of each peptide with the target growth factor has been confirmed, and the bioactivity of the immobilised growth factor has been demonstrated. Immobilised hTGF- $\beta 1$ have led to the activation of the signalling pathway and upregulation of collagen production in human hamstring derived cells. An upregulation of ALP content has been observed in response to the immobilised hBMP-2. Finally, the immobilised hVEGF has enhanced survival and proliferation of cultured HUVECs. When implanted subcutaneously in rats, implants functionalised with the growth factor binding peptides have captured the endogenous target growth factor, accumulating it around the implant which ultimately leads to a more pronounced tissue response when compared with the negative control. The results presented in this thesis demonstrate the potential of using synthetic peptides in the design and improvement of biomaterials, providing them with the potential to capture and immobilise growth factors and thus accelerate the healing process. To this end, gradients of growth factors that cover the length of a medical device have been 
fabricated; these types of gradients are envisioned to aid in the tissue reconstruction of injured ligaments to support the growth of ligament cells and bone integration. 


\section{Samenvatting}

Orthopedische verwondingen worden geassocieerd met pijn, invaliditeit en een grote financiële last voor de patiënt en de maatschappij. Pezen en ligamenten zijn slecht doorbloed en hebben daarom een laag herstellend vermogen. Een chirurgische ingreep is de meest voorkomende behandeling om het natuurlijke genezingsproces van deze weefsels na laceratie of breuk te begeleiden. Echter, in sommige gevallen is de schade zelfs na de chirurgische ingreep groter dan het natuurlijke herstelvermogen. Dit zal leiden tot een hersteld weefsel waarvan de biomechanische eigenschapen niet overeenkomen met die van het oorspronkelijke weefsel. Om de medische hulpmiddelen die geïmplanteerd zijn tijdens de chirurgische ingreep verder dan de huidige stand van zaken te ontwikkelen, moet de kennis van verschillende vakgebieden, zoals de scheikunde, oppervlakte- en interface-chemie, celbiologie, biomedische technologie en klinische praktijk, geïntegreerd worden om tot nieuw ontwikkelde implantaten te komen die strategieën integreren om groeifactoren leveren. Deze groeifactoren zijn cruciaal om de integratie van implantaten in weefsel te verbeteren. Groeifactoren zijn ook betrokken bij de activering en regulatie van verschillende cellulaire functies in alle stadia van het genezingsproces. Afgiftesystemen die de controle hebben over ruimtelijke en temporele presentatie van groeifactoren in het gewonde weefsel zijn daarom essentieel voor de ontwikkeling van strategieën ter verbetering van het genezingsproces.

Deze thesis beschrijft een nieuwe strategie om groeifactoren te immobiliseren en te leveren. Verscheidene biomaterialen zijn chemisch gemodificeerd en gefunctionaliseerd met synthetische peptiden, die specifiek interageren met verschillende groeifactoren, afhankelijk van de aminozuursequentie van de peptiden. Peptiden die met TGF- $\beta 1$, BMP2 en VEGF interageren zijn gesynthetiseerd en covalent geïmmobiliseerd op biomaterialen. De interactie van elk peptide met de gezochte groeifactor is bevestigd en de bioactiviteit van de geïmmobiliseerde groeifactor is aangetoond. De geïmmobiliseerde hTGF- $\beta 1$ heeft geleid tot de activering van signaalwegen en de opregulatie van collageenproductie in menselijke hamstring-afgeleide cellen. In reactie op de geïmmobiliseerde hBMP-2 is een opregulatie van de ALP-inhoud waargenomen. Tot slot heeft de geïmmobiliseerde VEGF de overleving en proliferatie van gekweekte HUVEC's verbeterd. Wanneer subcutaan geïmplanteerd bij ratten, implantaten gefunctionaliseerd met de groeifactor-bindende peptiden hebben de endogene gezochte groeifactor gevangen en heeft het rondom het implantaat geaccumuleerd wat uiteindelijk leidt tot een meer uitgesproken weefselreactie in vergelijking met de negatieve controle. De in deze thesis gepresenteerde resultaten tonen de mogelijkheid 
van het gebruik van synthetische peptiden in het ontwerp en de verbetering van biomaterialen aan om deze te voorzien van het potentieel om groeifactoren te vangen en te immobiliseren en daardoor het genezingsproces te versnellen. Daarvoor zijn gradiënten van groeifactoren gefabriceerd die betrekking hebben op de lengte van een medisch hulpmiddel. Dit soort gradiënten worden beoogd om de weefselreconstructie van gewonden ligamenten te bevorderen ter ondersteuning van de groei van ligamentcellen en botintegratie. 


\section{Acknowledgements}

During this period of three years I met several people that in one way or another contributed to this end. I could not finishing this book without having a final word to all of them.

I would like to start with my promotors. In first place I want to thank to Däniel for giving me the opportunity to work under your supervision in such interesting project. Your unique way of leadership always pushed me forward and gave me the confidence and chance to evolve as a person and as researcher. I want to thank you for giving me the opportunity to go for conferences and for setting up the collaborations I had during my PhD. Your passion and enthusiasm for science are inspirational and contributed to make me a better professional.

I also want to thank my second promotor Pascal. I really enjoyed this time within your group. Both individual and group meetings proved to be very informative and I really learned a lot during the time I spent with you. Your way of supervising the students: always informal, relaxing and with the door always open for everybody creates such a friendly environment within the group that I am really happy of being part of. Thanks for all the advices, suggestions, critics and support during these three years. I wish the best to you and your family - Dodo and Jasmin - and professionally as well.

Next, I want to thank my co-promotor Hugo. You were the first to introduce me to this position and one of the main reasons why I initially applied to it. I cannot thank you enough for all the help you gave me during the time I spent in Enschede. Thanks for your guidance, your tips and sharing your knowledge with me. Unfortunately, after a couple of months you moved back to Portugal. Even being thousands of kilometers away, you were always available to help me and to answer my doubts. That I will never forget. Thanks for all the time we spent outside work, either playing football or drinking beer. I wish you all the best in your professional and personal life - with Ana, Bernardo and Guilherme - and that Sporting is champion this year! Obrigado.

To my paranymphs: David, you were one of the most important persons I met since I arrived to Enschede. Thanks for everything, specially your friendship! I wish you all the best with Joana (to Joana: please take care of David). Gülistan, we shared the same table during our master thesis and then the same fumehood during the PhD thesis. Thanks for all moments at work and outside of it. Thanks for all the help, suggestions, work 
discussions and the nice stay in Boston. All the best during the remaining time of your PhD.

Next I want to thank to the members of the tendon and ligament group. I would like to start by thanking Corina for all the help and ideas during me PhD. Good luck in finishing your thesis. Eman, I really enjoyed sharing the office with you, it was so much fun. Thanks for visiting me in Portugal and allowing me to stay at your place in Boston and NY. I really had a great time with you in and outside the university, hope to see you again soon. To my students Joyce and Albert for their help and passion during the period they worked under my supervision. Thanks to Joyce for teaching me to count until tin in Dutch and to Albert for showing me that Dutch can actually play football. I also want to thank to Thijs, Stijn, Carlo, Maaike and Jére for their contribution to the group meetings and fun outside work. Wish you all the best in future. A specially thanks to Angel and Bruma for their tremendous effort during our collaboration. Thank you a lot for all the work with the in vivo experiments and I hope that we can continue working together in the future.

I want to thank all the members and ex-members of Pascal's group that I shared my time with - Jasper for all the teaching and discussions; if was not for you I would still be doing my PhD. Good luck with your company. I want thank to Tushar for all the help with HPLC and Emanuela and Jenny with the peptide synthesis. You were extremely helpful to me and I really learned a lot from you; thank you for teaching me how to get a peptide. I also want to thank to Bettina, Gülistan, Mark, Maike, Liulin, Shirish, Wies, Jen, Nicole, Aylin, Laura, Nora, Leendert and Eveline for your contribution during group meetings. Wish you all the best both professionally and personally. From the MnF, I want to thank to Marcel, Richard and Regine for being always available to help me and teach me regarding the laboratorial problems I faced during my PhD. Specially thanks to Marcel for setting up the dip coater device. Nicole and Izabel, thanks a lot for all the help during my PhD. Of course could not forget my teammates from the football team - Jacopo, He, Lorenzo, Ivan, Mark, Roberto, Andreas, Christoph and Andrea - it was a pleasure to share the pitch with all of you. Thanks to all the members and ex-members of the MnF that made my stay so much pleasant.

To Marcel for receiving me at the DBE group and for accepting to be part of my committee. I had a great time within your group and really enjoyed the group meetings. I want to thank to my long time office mate Brenda and more recently Bram for all the moments in the office. I want to thank to Ingrid for all the help during my stay in DBE and in the graduation process. Jacqueline for all the help and keeping the order in the labs. To the members and ex-member of the DBE: Piet, Janneke, Aart, Jeroen, Jannine, Sanne, 
Maurice, Yao, Kannan, Elahe, Jan, Sieger, Tom, Lisanne, Milou, Mijke, Rong, Leilei, Lorenzo, David, Michelle, Jorke, Vasillis, Cindy, Shaun, Taciana and of the AMBER group: Séverine, Jean-Baptiste, Adithya, Bárbara, Miguel and Darya for all the time we shared together during and outside work.

Of course I could not forget my first group, the Tissue Regeneration. I had a great time there during my master thesis and part of my PhD. I want to thank to professor Clemens for giving me the opportunity to work within his group. Nicolas and Erik for accepting me as your student for my master project. Specially thanks to Erik, my daily supervisor, for teaching me everything and helping during my stay in Enschede. Ivan, for sharing the office with me - it was a pleasure tío and I wish you all the best in the future. To the Portuguese crew from the group - Carlos (thanks for all the motivation); Ana for all the food you cooked and giving me shelter at your place, dá cá dois beijos; Afonso, meu puto; Daniel, Danielle, Minês, Paulo, Pedro, Cristina, Catarina, Luís, David, Filipa, Rita, Diogo, Tiago, Inês, Sara for all the great time we had together and all the Portuguese dinners. I also want to thank to Lorenzo, Febryani, Paul, Angad, Honglin, Alexei, Nick, Ziryan, Tom, Audrey, Anne, Giulia, Bach, Elena, Federico, Barbara, Miriam, Nathalie, Niloofar, Simone, Nazli, Ester, Jan, Pamela, Vanessa, Roman, Anouk, Andrea, Giuseppe, Alessia, Afrodite for the useful discussions and the great time outside work.

I want to thank to all my housemates. Björn, thank you for opening the doors of your house and for all the good time we shared at PPP. Thank you Inês for all the time we shared in Enschede. Vanessa, Denisa, Bojan, Riccardo and Alex for making PPP such a nice place to live. During my time in Enschede also meet a lot of people outside work: thank you Joana for being the best host ever, for the delicious dinners and of course selecting me all the time to wash the dirty dishes; Francisco $G$ and Roman, thanks a lot for all the moments; to all the members of the UTKring for all the football matches on Mondays; Eduardo, Lionel, Inês, Ana, Mohammed, Juan, Kasia, Gerard, Nana and Maddy for making my stay in Enschede much funnier. I could not forget my friends from Portugal, that kept in touch with during all this time. Thanks to my home boys - Cruz, Felipe, Cópio, Ruby, Zeka, Lourido. To André for giving me place every time I went to Amsterdam. I also want to thank to my friends Marcelo and Parruca for coming all the way from Delft to visit me. Minês, Teresa, Catarina, Aguiar, Belo, Sá, Gui, MJ, Marilena and Ricardo thanks for everything.

Last but not least to my family, specially my mother, father and brother. Thanks for all the life time support, help and always believing in me. I would not be here today if it was not for you. 


\section{About the author}

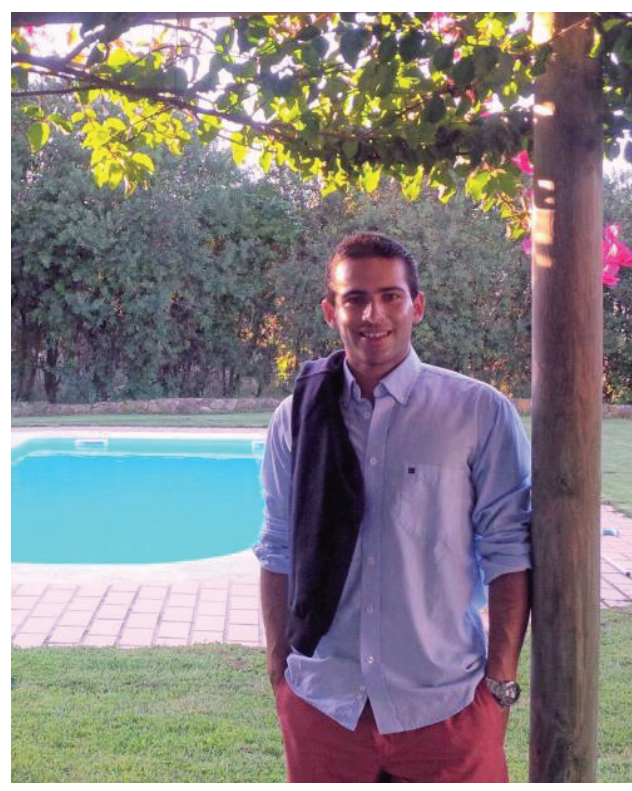

João was born in Beja (Portugal) and studied biological engineering (an integrated master's degree) at the Superior Technical Institute in Lisbon.

His master's project was performed in the Tissue Regeneration department of the University of Twente during an exchange as an Erasmus student. The project consisted of a high throughput screening platform for embryonic development, and the goal of the thesis was to study and increase the formation of the artificial stage of the morula. During the summer of 2012, João also worked as a trainee on a project for the improvement of the quality of cork in the Agrogenomics Research Group at CEBAL.

In June of 2013, João joined the groups of Prof. Dr. Däniel Saris and Dr. Hugo Fernandes (Tissue Regeneration) and Prof. Dr. Pascal Jonkheijm (Molecular Nanofabrication) as a PhD candidate at the University of Twente. João recently accepted a Post-doc position in the University of Twente under the supervision of Prof. Dr. Däniel and Prof. Dr. Pascal Jonkheijm. 


\section{This thesis is based on the following publications:}

- Ekwueme EC, Shah JV, Mohiuddin M, Ghebes CA, Crispim JF, Saris DB, Fernandes HA, Freeman JW. Cross-Talk Between Human Tenocytes and Bone Marrow Stromal Cells Potentiates Extracellular Matrix Remodeling In Vitro. Journal of Cell Biochemistry 117(3) (2016) 684-693.

- $\quad$ Crispim JF, Lee YW, Fu SC, Fernandes H, Jonkheijm P, Saris DB. TGF-ß1 activation in hamstring cells through growth factor-binding peptides on polymers. (Submitted)

- $\quad$ Crispim JF, Lee YW, Fu SC, Fernandes H, Jonkheijm P, Saris DB. Immobilization of hBMP-2 on polymers directs cell fate. (In preparation)

- $\quad$ Crispim JF, Lee YW, Fu SC, Fernandes H, Jonkheijm P, Saris DB. Improvement of medical devices with VEGF-binding peptides in order to induce vascularization in orthopaedic injuries. (In preparation)

- Crispim JF, Fernandes H, Jonkheijm P, Spatially presenting hTGF- $\beta 1$ on biopolymeric films. (In preparation) 

